\title{
NISTIR 4478
}

NEW NIST PUBLICATION

April/May 1991

\section{Recommended Fine}

Positioning Test for

the Development Test

Fight [DTF-1] of the

NASA Fight

Telerobotic Servicer

[FTS]
Nicholas Dagalakis

Performance Measures Group

Albert J. Wavering
Intelligent Controls Group

U.S. DEPARTMENT OF COMMERCE

National Institute of Standards

and Technology

Robot Systems Division

Bldg. 220 Rm. B124

Gaithersburg, MD 20899

\section{Peter Spidaliere}

National Aeronautics and Space Administration Goddard Space Flight Center

Greenbelt, MD 20771

U.S. DEPARTMENT OF COMMERCE Robert A. Mosbacher, Secretary NATIONAL INSTITUTE OF STANDARDS AND TECHNOLOGY

John W. Lyons, Director 



\section{Recommended Fine Positioning Test for the Development Test Fight [DTF-1] of the NASA Flight Telerobotic Servicer [FTS]}

Nicholas Dagalakis Performance Measures Group

\section{Albert J. Wavering Intelligent Controls Group}

U.S. DEPARTMENT OF COMMERCE National Institute of Standards and Technology Robot Systems Division Bldg. 220 Rm. B124

Gaithersburg, MD 20899

\section{Peter Spidaliere}

\author{
National Aeronautics and \\ Space Administration \\ Goddard Space Flight Center \\ Greenbelt, MD 20771
}

U.S. DEPARTMENT OF COMMERCE Robert A. Mosbacher, Secretary NATIONAL INSTITUTE OF STANDARDS AND TECHNOLOGY

John W. Lyons, Director 



\section{Table of Contents}

List of Figures

List of Tables

1.0 Introduction

2.0 Statement of the Problem

3.0 Definitions and Discussion

4.0 Equipment Set-up, Test Procedures, Analysis and Results

4.1 Introduction

4.2 Equipment Set-Up

4.3 Test Procedures

\subsubsection{Teach Mode Control Tests}

4.3.1.1.a Teach mode joint angles kinematics control

4.3.1.1.b Analysis and Conclusions

4.3.1.2.a Teach mode inverse kinematics control

4.3.1.2.b Analysis and Conclusions

4.3.2 Coordinates Transformation Test

\subsection{2.a Test}

4.3.2.b Analysis and Conclusions

\subsubsection{Off-Line Programming Tests}

4.3.3.1.a Standard initial positions off-line programming

4.3.3.1.b Analysis and Conclusions

4.3.3.2.a Limited motion off-line programming

4.3.3.2.b Analysis and Conclusions

4.3.4 Robot Position Resolution Tests

4.3.4.a Tests

4.3.4.b Analysis and Conclusions

4.4 Forward Kinematics Error Analysis

4.4.1 Continuous operation error analysis and Conclusions

4.4.2 Interrupted operation error analysis and Conclusions

5.0 Conclusions

6.0 Recommendations for Further Investigation

7.0 References

8.0 Acknowledgements

9.0 Appendix 



\section{List of Figures}

3.1 Test results demonstration plot.

4.1 Schematic of the equipment set-up.

4.2 Location of sensor nest \#2.

4.3 Relative locations of coordinate systems.

4.4 Teach mode joint angles kinematics control positions plot.

4.5 Teach mode joint angles kinematics control positions plot.

4.6 Position accuracy error plot.

4.7 Position repeatability error plot.

4.8 Measured position coordinate plot.

4.9 Measured position coordinate plot.

4.10 Measured position coordinate plot.

4.11 Teach mode inverse kinematics control positions plot.

4.12 Teach mode inverse kinematics control positions plot.

4.13 Position accuracy error plot.

4.14 Position repeatability error plot.

4.15 Measured position coordinate plot.

4.16 Measured position coordinate plot.

4.17 Measured position coordinate plot.

4.18 Coordinates unansformation analysis plot.

4.19 Coordinates taansformation analysis plot.

4.20 Standard initial positions off-line programming positions plot.

4.21 Standard initial positions off-line programming positions plot.

4.22 Position accuracy error plot.

4.23 Position repeatab lity error plot.

4.24 Measured position 'oordinate plot.

4.25 Measured position c ordinate plot.

4.26 Measured position cc :rdinate plot.

4.27 Limited motion off-lire programming positions plot.

4.28 Limited motion off-line programming positions plot.

4.29 Position accuracy error plot.

4.30 Position repeatability error plot.

4.31 Measured positi: in coordinate plot.

4.32 Measured posit on coordinate plot.

4.33 Measured position :oordinate plot 
4.34 Measured position coordinate plot.

4.35 Measured position coordinate plot.

4.36 Measured position coordinate plot.

4.37 Robot resolution test positions.

4.38 Plot of the measured magnitudes of the increments.

4.39 Plot of the measured magnitudes of the increments.

4.40 Plot of the measured magnitudes of the increments.

4.41 Robot resolution test positions.

4.42 Forward kinematics (continuous operation).

4.43 Position accuracy error plot.

4.44 Position repeatability error plot.

4.45 Forward kinematics (continuous operation).

4.46 Position accuracy error plot.

4.47 Position repeatability error plot. 


\section{List of Tables}

4.1 Teach mode joint angles kinematics control analysis results.

4.2 Teach mode inverse kinematics control analysis results.

4.3 Coordinates transformation analysis results (14 positions).

4.4 Coordinates transformation analysis results (7 positions).

4.5 Standard initial positions off-line programming analysis results.

4.6 Limited motion off-line programming analysis results.

4.7 Robot position resolution analysis results (commanded incremental moves of 0.15 $\mathrm{mm})$.

4.8 Robot position resolution analysis results (commanded incremental moves of 0.5 $\mathrm{mm})$.

4.9 Forward kinematics error analysis results (continuous operation).

4.10 Forward kinematics error analysis results (interrupted operation). 


\subsection{STATEMENT OF THE PROBLEM}

The purpose of this report is to standardize definitions and propose test procedures for the DTF (Development Test Flight)-1 positioning tests.

The DTF-1 mission poses a number of technical problems never encountered during earth based robot performance measurements. First, although the design the DTF-1 calibration position sensor has not been decided yet it is expected that it will have a working volume smaller than the off-line programming accuracy of the robot. Second, thermal shifts during the test will cause distortions of the manipulator and sensor, possibly disturbing the tests. Regardless of the technical difficulties, the DTF-1 mission offers opportunities to understand the positioning capabilities of robots in the environment of space.

This report provides the framework about which the DTF-1 flight procedures can be developed to conduct the required fine positioning tests. First, there will be a discussion of the unique problems associated with the DTF-1 mission. This will include a standardization of terminology. Next, a very detailed description of flight-like testing, conducted at NIST, will be presented. Although the experiments conducted were performed on a robot of different design than the DTF-1, the results and techniques used can be extended to the mission. Finally, conclusions and possible future work will be presented.

\subsection{DEFINITIONS AND DISCUSSION}

In order to avoid confusion, the terminology and definitions for the fine positioning test must be standardized. Terminology and test procedures which were established by the ANSI/RIA (American National Standards Institute / Robotic Industries Association) R15.05 Robot Performance Subcommittee [RIA 90], and/or the ISO (International Organization for Standardization ) Industrial Automation Systems Technical Committee ISO/TC 184 [ISO 90] will be maintained throughout this report whenever it is possible. This terminology differs from that in the NASA Requirements Document (SS-GSFC-0043) in the following general ways: 
SS-GSFC-0043:

Accuracy - The difference between the actual position of the tool plate and the commanded position in Cartesian space.

Repeatability - The difference between the actual position of the tool plate and the commanded, previously taught position.

ISO:

Unidirectional pose accuracy expresses the deviation between a command pose and the mean of the attained poses when approaching the command pose from the same direction.

Unidirectional pose repeatability expresses the closeness of agreement between the positions and orientations of the attained poses after $\mathrm{n}$ repeated visits to the same command pose.

\section{ANSI/RIA:}

Static position accuracy is a statistical measure of the spatial deviation between commanded and achieved robot positions.

Positional repeatability is the measure of deviations between achieved robot positions and the mean of those positions after ordering the robot to the same pose $\mathrm{N}$ times from the same direction.

The term "pose", used in the above definitions, stands for position and orientation. The terms "attained" and "achieved" also used are equivalent. The term "tool plate" stands for the end of the robot arm tool mounting plate. The position or pose of the robot could be commanded by either off-line programming or teach mode control, depending on the needs of the application. Each of these modes of operation results in significantly different accuracy and repeatability errors.

The physical meaning of these definitions can be explained by using the demonstration plot of Figure 3.1. In this figure it is assumed that the test specifies that the robot moves to a single commanded position represented by point $C$. In the interest of simplicity we assume 


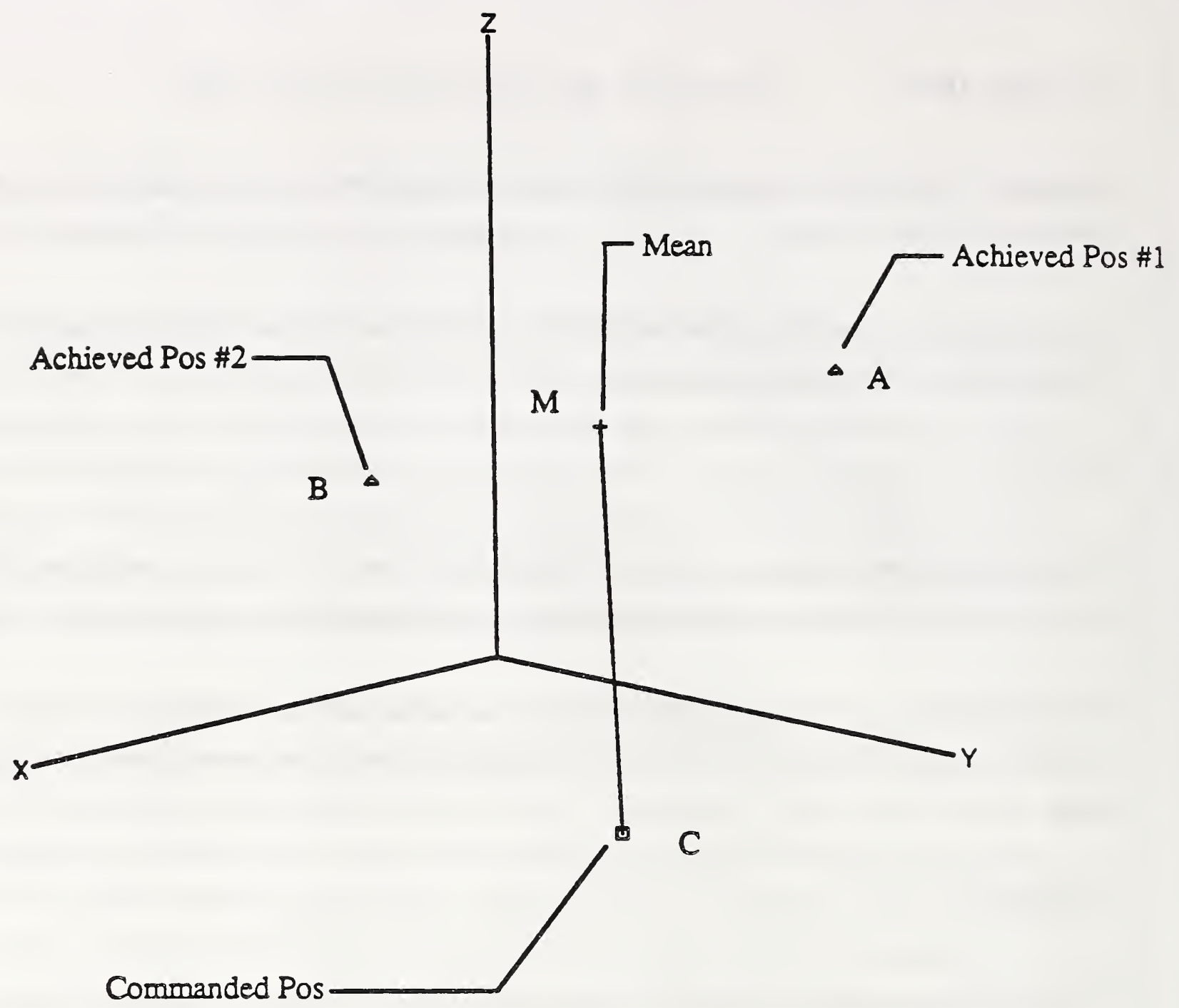

Plot of the PTP Test Achieved Positions

Each triangle is located at an achieved position

The square marks the commanded position

The cross marks the mean of the achieved positions

Figure 3.1 Test results demonstration plot. 
here that the robot makes only two attempts to reach that point. During its first attempt to reach that point the robot goes to point $A$ and during its second attempt goes to point $B$. The mean of those two achieved positions lies at the middle of the $\underline{A B}$ vector designated by point $\mathrm{M}$ in the figure. The positioning accuracy as defined by ISO, for this example, is the magnitude of the vector (CM) given by equations 4.5 and 4.9 in the next section. The positioning accuracy as defined by ANSI/RIA, for this example, is the mean of the magnitudes of the vectors $(\underline{C A})$ and $(\underline{C B})$ given by equations 4.2 and 4.3 in the next section. The positioning repeatability as defined by ISO, for this example, is the radius of a sphere with center $M$ given by equations 4.13 and 4.12 in the next section, which bounds points $A$ and $B$. The positioning repeatability as defined by ANSI/RIA, for this example, is the mean of the magnitudes of the vectors $(\mathrm{MA})$ and $(\underline{\mathrm{MB}})$ given by equations 4.10 and 4.11 in the next section.

The SS-GSFC-0043 definition of accuracy matches the generalized ISO/RIA definition of accuracy under off-line programming and the SS-GSFC-0043 definition of repeatability matches the generalized ISO/RIA definition of accuracy under teach-mode control. Additionally, the generalized ISO/RIA repeatability provides information regarding the statistical behavior of the data gathered during the test.

The objective of the ISO and the ANSI/RIA proposed tests is to cover as much of the robot workspace used during common industrial applications as possible. ISO proposes to fit a cube in the workspace of the robot which is to be tested and then use five points located on one of the diagonal planes of that cube as the test commanded positions. ANSI/RIA proposes the use of the vertices of a standard test path, which is defined by the standard, as the test commanded positions. The test results from all of these commanded positions should be averaged to obtain more representative values of accuracy and repeatability. In the case of DTF-1 only one test commanded position can be used, that of the sensor nest position, and its location is dictated by considerations other than those of these standards.

The ANSI/RIA test specifications require that off-line programming is used to match the test equipment (metrology instrument) coordinate system with the robot base coordinate system for the measurement of accuracy. The ISO test specifications do not have this requirement. Ideally the accuracy capability of a robot under either off-line programming or teach mode control should be measured and be known for the portion of its workspace with the greatest anticipated use. Due to the nature of the sensors to be used during the mission to measure the fine positioning characteristic of the DTF-1 robot, a modified 
definition of off-line programming accuracy is required. This is due to the fact that the working volume of the sensor nest is smaller than the volume of the cluster of points and the commanded point which would result from a standard off-line accuracy test. In other words, if the robot is commanded to a Cartesian position inside the nest it could arrive outside the working volume of the sensor and, therefore, the position would not be measurable. Because of this, a modified definition of off-line programming accuracy, forward kinematics accuracy, is required. Additionally, the operator will have to initially guide the manipulator into the sensor working volume since a computer command to the location will not necessarily deliver the tool plate to the sensor working volume.

Typically, off-line programming accuracy is measured by commanding the robot to a position and measuring the difference between that command and the actual position measured by some sensor. This technique could be thought of as measuring the inverse kinematic accuracy.

GSFC (Goddard Space Flight Center) and NIST (National Institude of Standards and Technology) have developed another technique by which the same basic information can be gathered. The technique is to send the robot to a position within the working volume of the sensor to measure the tool plates actual position and simultaneously query the robot regarding its perceived position. We call this the forward kinematic accuracy. If there is good agreement between the forward and inverse kinematic solutions, the off-line accuracy using this technique should be approximately the same as the standard, inverse kinematic technique. The result will be approximate since the standard test combines the two sources of error, errors due to inaccurately modeled kinematics and errors due to servo control inaccuracies. The forward kinematic, off-line programming accuracy includes only kinematic modelling errors, which should dominate the DTF-1 errors. The two techniques should agree everywhere within the workspace except near singularities where inverse kinematic equations are not well behaved. Because of the limited range of the sensor, the forward kinematic approach will be taken during the DTF-1 mission.

In addition to what SS-GSFC-0043 refers to as accuracy and repeatability, the document specifies incremental motion requirements. Incremental motion is not defined by either ISO or RIA but is commonly referred to as resolution. For the purposes of this report incremental motion shall be defined as the smallest controllable Cartesian displacement and orientation change of the manipulator tool plate coordinate frame with respect to the 
manipulator base coordinate frame. Incremental motion shall be calculated using the same set of equations specifying accuracy.

\subsection{EQUIPMENT SET-UP, TEST PROCEDURES, ANALYSIS AND RESULTS}

\subsection{Introduction}

The objectives of the experimental work were to simulate the FTS performance test procedure and to develop analysis and display software. Since neither the FTS robotic arm nor the sensor nest are currently available, it was decided to use robot equipment with performance characteristics similar to those specified for FTS.

A variety of test procedures were simulated and a large number of data were collected. Based on the results of the analysis several of these tests were repeated and test conditions were modified in order to clarify questions which were raised by the analysis of the data.

For the analysis of the data and display of the results a NIST robot testing and calibration workstation was used. The workstation was interfaced with the controllers of the robot arm and the metrology instrument used in order to facilitate the collection and exchange of data. New software had to be written and old robot performance analysis programs had to be modified in order to satisfy the peculiarities of the FTS tests.

This section is organized as follows. First, the experimental equipment used to conduct the simulated performance tests is described. This is followed by a discussion of some general procedural aspects which were common to all tests. Next, details of individual test procedures, results, and analyses are presented.

\subsection{Equipment Set-Up}

The simulated FTS performance tests were performed in the lab of the Intelligent Controls Group (ICG) at NIST. The following equipment was used to perform the tests: 
Robotics Research Corp. (RRC) K-16071 dextrous manipulator and controller

NIST robot control system target hardware (VME backplane and boards)

NIST control system software development and user interface workstations (Sun

3/160's)

Automated Precision, Inc. (API) Smart 310 laser tracker metrology system

Robot testing and calibration workstation (Macintosh II)

The equipment and system interconnections are shown in Figure 4.1. Each piece of equipment is discussed in detail below.

The RRC K-1607 is a 7 degree-of-freedom kinematically redundant manipulator. The manipulator base is mounted at 45-degrees to the floor, as shown in Figure 4.1. The K1607 drive system consists of permanent-magnet dc motors with harmonic drive gear reduction. Position and velocity feedback are provided by brushless resolvers driven by anti-backlash gearing. The resolvers measure the joint output position, rather than the motor shaft position. An integral torque sensor on each joint provides output torque information which is used in a feedback loop to minimize the effects of drive nonlinearities (friction and compliance in particular). A Servo Level Interface is provided by RRC which allows an external computer system to issue joint torque, position, velocity, or motor current commands to the manipulator every $2.5 \mathrm{~ms}$ [Eissmann 89]. Position, velocity, and torque feedback values updated at this rate are also available. Servo Level Interface variables may be accessed via common memory locations on the Multibus backplane which resides in the $R R C$ controller.

All motions performed during the tests were generated and controlled by the NASREM (NASA/NBS Standard Reference Model for Telerobot Control) control system being developed by the Intelligent Controls Group (ICG) at NIST [Albus 87, Fiala 89a]. This control system is being implenented in Ada, and is based on the concept of a hierarchical organization of redistributable cyclically-executing processes which communicate via common memory buffers. The system runs on (currently) five Motorola 68020-based single-board computers which reside in a VME backplane. A high-speed (225 kbaud) serial link is used to transfer command and feedback information between the ICG control

1 "Certain commercial equipment, instruments or materials are identified in this paper in order to adequately specify the experimental procedure. Such identification does not imply recommendation or endorsement by the National Institute of Standards and Technology, nor does it imply that the materials or equipment identified are necessarily the best available for the purpose". 


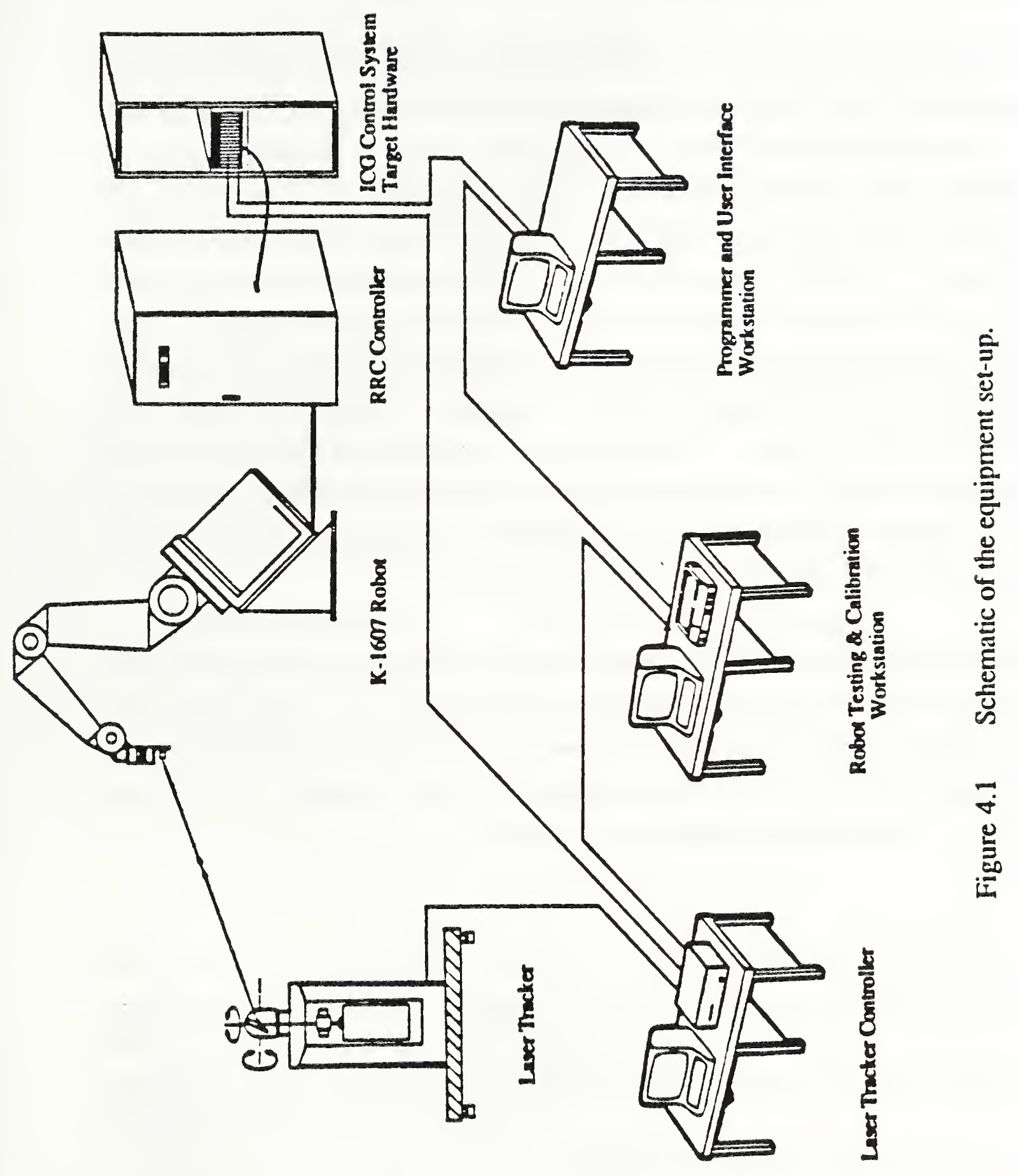


system and the RRC backplane on a 5 ms basis (ie, every other RRC cycle) [Fiala 89b]. Sun 3 workstations are used for software development and for user interaction with the system during runtime. Communication between the Sun(s) and the target boards for keyboard and file i/o takes place over 9600 baud serial lines.

The current implementation of the NASREM hierarchy consists of Primitive (trajectory generation) and Servo levels. Detailed information about the design of these levels may be found in [Wavering 88, Fiala 88]. These levels provide a number of different algorithms for trajectory generation and manipulator servoing. Two different trajectory generation algorithms are used for metrology test motions; one for joint space motions, and one for Cartesian straight line motions. For motions to goal poses specified in terms of desired joint positions, joint-interpolated quintic polynomial trajectories are used [Craig 86]. Joint space trajectory functions are evaluated every $5 \mathrm{~ms}$. For Cartesian goal poses (represented as a 3 dimensional position vector and a quaternion rotation), quintic polynomial functions of the Cartesian variables are used. Cartesian trajectory functions are evaluated every 25 ms. The Cartesian trajectory points are transformed into joint space before commanding them to the Servo level. An inverse kinematics algorithm based on the augmented Jacobian [Seraji 89, Kreutz 89] is used to perform this transformation.

Although other algorithms are available, a high-gain individual joint PID servo was used for all of the metrology tests. This algorithm was used because of the high stiffness and disturbance rejection it provides. Gravity compensation torques are added to the errorbased torques to counteract the effects of link masses in a 1-g environment. The following control equation is computed for each joint, each cycle of Servo execution:

$\tau=K_{p}\left(\theta_{d}-\theta\right)-K_{v} \dot{\theta}+K_{i}\left(\int \theta_{\text {err }}\right)+\tau_{\text {grav }}$.

where $\tau=$ torque commanded to robot, $\mathrm{K}_{\mathrm{p}}, \mathrm{K}_{\mathrm{v}}, \mathrm{K}_{\mathrm{i}}$ =position, velocity, and integral gains, $\theta_{\mathrm{d}}=$ desired joint position, $\theta=$ actual position, $\dot{\theta}=$ actual joint velocity, $\int \theta_{\text {err }}=$ integral of position error, $\tau_{\text {grav }}=$ gravity compensation torque.

There is no deadband in the algorithm; that is, every error, no matter how small, is multiplied by the appropriate gain to determine a correcting torque. However, residual Coulombic friction outside the torque loop (bearing and seal friction) prevents very small 
torques from causing arm motion. Except where otherwise noted, the following gains were used for all metrology tests:

$\mathrm{K}_{\mathrm{p}}=\operatorname{diag}[3000030000160001250017002000500] \mathrm{N}-\mathrm{m} / \mathrm{rad}$

$\mathrm{K}_{\mathrm{v}}=\operatorname{diag}[800800350250708050] \mathrm{N}-\mathrm{m}-\mathrm{s} / \mathrm{rad}$

$\mathrm{K}_{\mathrm{i}}=\operatorname{diag}[1000100050050017020050] \mathrm{N}-\mathrm{m} / \mathrm{rad}-\mathrm{s}$

These gains were determined experimentally. The around-the-loop time for this algorithm, including communication time, is $10 \mathrm{~ms}$ (although commands and feedback are updated every $5 \mathrm{~ms})$.

The system has a simple user interface which allows motion commands to be specified from the keyboard or from a data file. The command information for the metrology test motions includes the following:

\section{Command parameter}

Trajectory algorithm

Goal pose

Redundancy resolution

Traversal time

\section{Comments on use for metrology tests}

joint_quintic or Cartesian_quintic

desired joint positions or end plate Cartesian position and orientation with respect to base coordinates

Cartesian_quintic only; specifies to use the augmented Jacobian-based inverse kinematics along with the desired elbow plane angle desired duration of motion

For each motion, the user can also indicate whether or not position information is to be recorded when the motion is complete. If the final position is to be recorded, the user interface process delays for $1 \mathrm{~s}$, reads the joint and Cartesian feedback buffers, stores this information, signals the laser tracker system to record data for the point, and delays for $2 \mathrm{~s}$ before continuing to the next command.

The position of the origin of the robot arm mechanical interface coordinate system, located on the end-of-arm mounting plate, was monitored with a laser tracker metrology system [K. Lau 85, API 90]. This system can direct a laser beam to a retroreflector target and determine its three dimensional space spherical coordinates, using an interferometer and 
precision encoders. As the target is moving, the laser tracker servoes the mirror which reflects the laser beam to keep it pointing on the target all the time. As long as the beam stays within the acceptance angle of the target, and the speed and acceleration of the target do not exceed certain limits set by the laser tracker servo-drive system and controller, the target is continuously tracked. The controller of the laser tracker can be directed to continuously sample and save the position coordinates of the retroreflector target at a frequency of up to $450 \mathrm{~Hz}$, or sample only when directed to do so. The sample command signal can come from the keyboard or from a direct connection to an external controller.

A hollow cornercube retroreflector target was used for all the tests. A special fixture was built in order to mount the target to the interface plate of the robot arm (end-of-arm plate). The fixture had a weight of $1.715 \mathrm{Kg}(3.773 \mathrm{lb})$, an axial offset of approximately $25 \mathrm{~mm}$ ( 1 in) and a radial offset which was essentially $0 \mathrm{~mm}(0 \mathrm{in})$. A 90-degree angle bracket was also built and used for the dimensional calibration of the target mounting fixture. An aluminum calibration bar was used for the initialization of the laser tracking system. Two target mounting locations were machined on the bar and their distance was measured with a coordinate measurement machine. The bar was clamped in a fixed location close to where measurements would be made.

A newly-developed robot testing and calibration workstation was used to analyze the data . The workstation has several basic communication programs and two ports, which allow it to exchange commands and data with robot and the laser tracker controllers. Ordinarily the workstation is connected to the communication ports of the robot and laser tracker controllers and coordinates the test activities. This is usually done by commanding the robot to execute the command programs required at each stage of the test and then waiting until the robot controller acknowledges the completion of the execution of these commands. The workstation either collects continuously sampled position data from the laser tracker controller or commands it to sample and store such data. Once that is completed any necessary processing of the data is done and the next step of the test is initiated. The robot command programs usually reside in the robot controller, so that the workstation action only involves their activation, thus minimizing the possibility of unpredictable robot behavior due to bad communications.

In the present set-up, however, it was decided to have the robot control system initiate all data collection actions via a direct connection to the metrology instrument controller. Although this means that the software used to conduct the test and record points is very 
robot specific, it was felt that it would more accurately simulate the way the FTS control system will perform these tests in space. In addition, the time delay between the end of the robot arm motion and the measurement of the position of the target is somewhat reduced with this approach. As mentioned in the discussion of the robot control system, each time the laser tracker controller was signalled to store a point, the robot control system also stored joint position and target Cartesian position information computed using forward kinematics. This robot control system position data was sent to the robot testing and calibration workstation after test completion via a serial line connected between the Macintosh II and the Sun 3 workstations.

\subsection{Test Procedures}

In designing the FTS performance test procedures it was desired to follow those recommended by the ANSI/RIA (American National Standards Institute / Robotic Industries Association) R15.05 Performance Subcommittee [RIA 90], and/or the ISO (International Organization for Standardization ) Industrial Automation Systems Technical Committee ISO/TC 184 [ISO 90]. Unfortunately this was not possible because of the constraints of the present FTS environment, particularly the requirement that the position and orientation (pose) of the end-effector is measured at only one or two specific locations in the robot workspace where sensor nests will be located. Thus, only basic ideas from the two standard tests were used. Both the RIA and the ISO static PTP (Point-to-Point) accuracy and repeatability tests require the robot to move to various measurement positions, which are specified for the workspace of each robot, and the achieved poses are measured by appropriate robot metrology instruments. Each committee has selected a different set of points. The standard path between those points is also different, and the orientation can be random or fixed depending on the type of the test.

In the present test study only one measurement position was used, instead of the several positions specified by [RIA and/or ISO 90], because of the sensor nest limitations mentioned previously. The coordinates of the sensor nest location used for the current tests, with respect to the baseframe of the FTS arm, were provided by the FTS contractor and are shown in Figure 4.2. Although there was no actual sensor nest, all measurements were taken with the manipulator in the vicinity of the location where a sensor nest would be if the RRC robot were mounted horizontally (instead of at 45 degrees) as the right arm of the FTS. This position will be referred to as the simulated sensor nest or S2 in the 


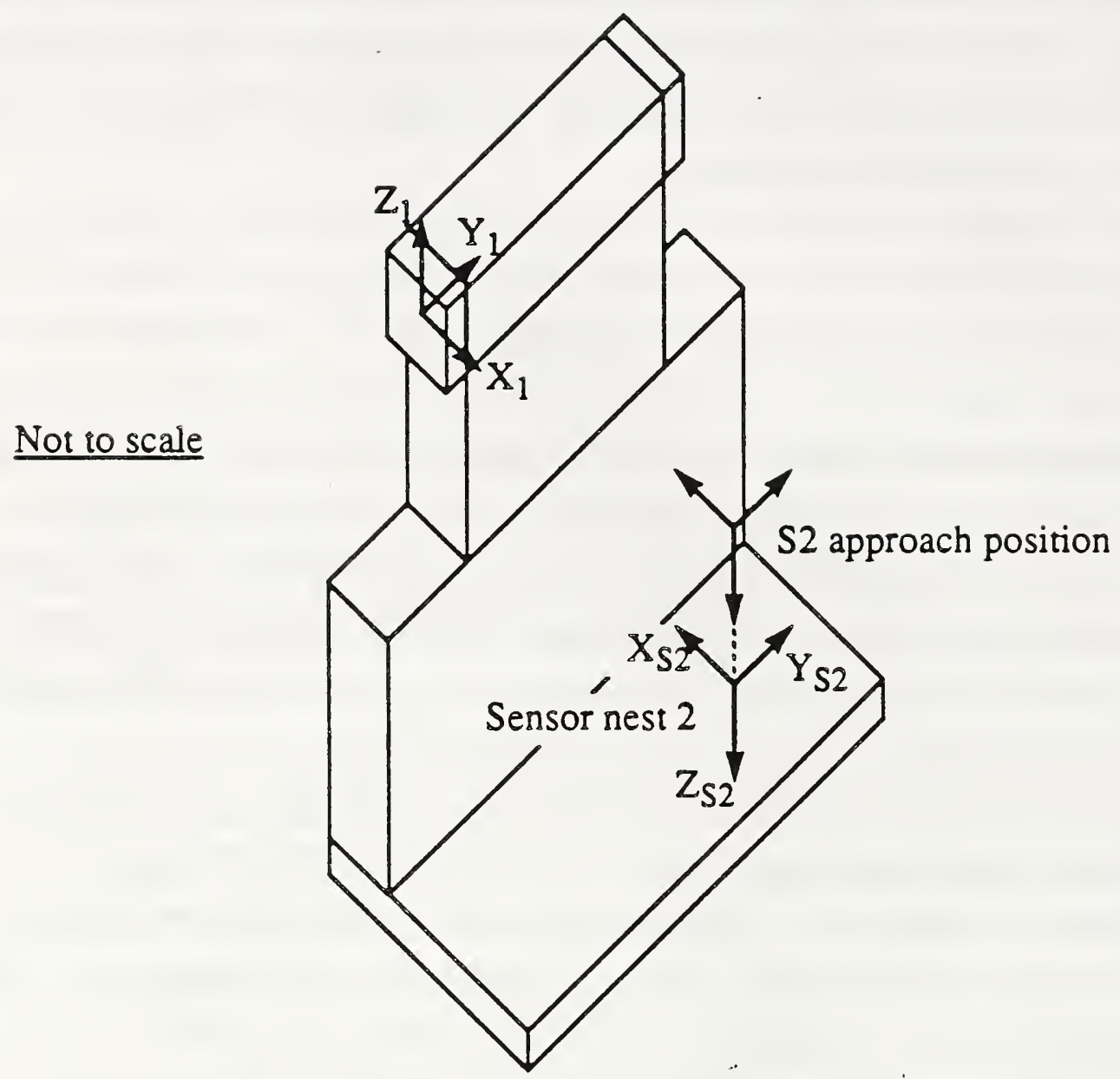

S2 location (with respect to $\left.X_{1} Y_{1} Z_{1}\right):(14.0,28.0,-43.5,+180.0,0.0,+180.0)$ (in, deg)

Figure 4.2. Location of sensor nest \#2. 
descriptions of the tests. Another position used for many of the tests is an approach point for the simulated sensor nest. At this point the robot arm interface plate has the same orientation as at $\mathrm{S} 2$, and is displaced about $0.46 \mathrm{~m}$ (18 in) along the $-\mathrm{Z}$ axis of the $\mathrm{S} 2$ coordinate frame. At the time these tests were performed, the design of the sensor nest dictated that such an approach point be used in moving into and out of the nest. The S2 approach position will be referred to as $S 2_{\text {app }}$.

Although the RIA and ISO standard test positions were not used as measurement locations, they were used in many of the accuracy and repeatability tests as starting positions for motions that ended at the simulated sensor nest. These initial positions were used to assess how well the robot could move to a particular location from different areas of the workspace. The arm was commanded to move sequentially from each one of the vertices of the RIA standard path to the approach point, and from there to the measurement position. The RIA measurement positions were chosen instead of the ISO measurement positions as more representative of the positions where FTS will perform most of its work. The coordinates of the vertices of the RIA standard path were calculated based on a simple robot workspace size measurement test and the specifications provided by [RIA 90]. As with $S 2$ and $S 2_{\text {app }}$, these positions were transformed to account for the 45-degree mounting of the robot. Only eight of the standard test positions, out of twelve, fell within the workspace of the robot used and were used for the tests. The same end plate orientation was used at each of the standard positions.

A variation of this procedure was used for some tests, in which the robot was commanded to move directly from $S 2_{\text {app }}$ to $S 2$ and back for each of eight repetitions, without using the RIA positions as starting locations. This alternative was examined out of concern that the test which uses the standard test positions would take more time than is available.

The laser tracker was placed at a distance of approximately $2000 \mathrm{~mm}$ from the simulated sensor nest location. Figure 4.3 shows the relationship between the reference coordinate systems of the robot and the laser tracker. Although the $\mathrm{Z}$ axis of both coordinate systems is in the same direction, the $X-Y$ plane of the laser tracker coordinate frame is about 450 $\mathrm{mm}$ above the robot base coordinate frame. Also shown is the relative X-Y location of the simulated sensor nest, $S 2$. The $Z$ position of the laser tracker target at $S 2$ is about $620 \mathrm{~mm}$ below the $X-Y$ plane of the robot base coordinate frame. During the tests the distance between the target, which was mounted on a fixture attached to the robot arm interface plate, and the laser tracker head varied from approximately $1500 \mathrm{~mm}$ to $3500 \mathrm{~mm}$. 


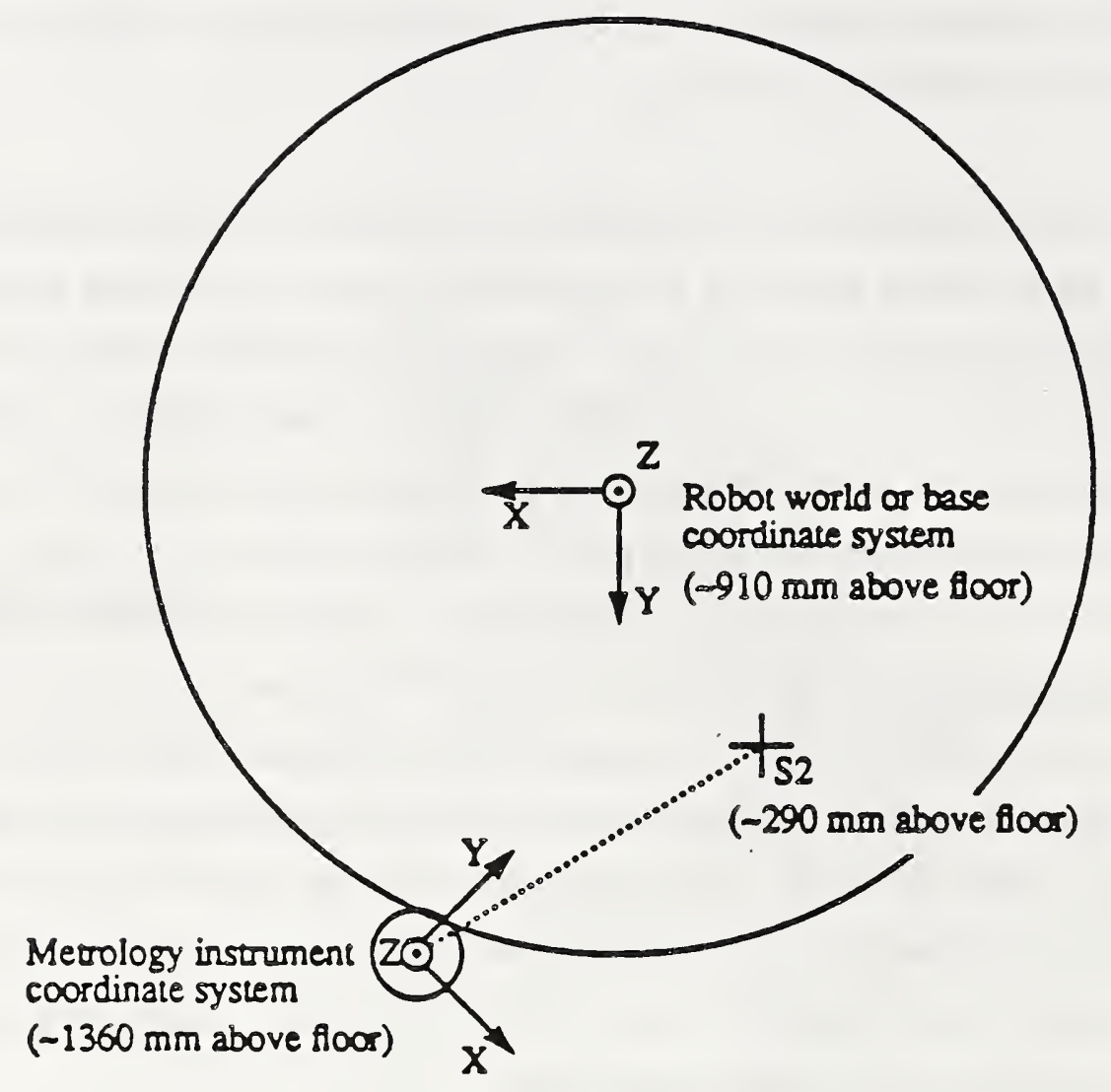

Figure 4.3 Relative locations of coordinate systems. 
The laser tracker was initialized, before its use, with the help of the calibration bar. This was done in the following way. The laser beam was first locked on the target, then the target was moved from one of the bar target mounting locations to the other and the known distance was communicated to the laser tracker controller. Based on that information the controller calculated the radial distance to the two bar target mounting locations. The target could then be moved to its mount at the end of the arm for the performance test. As long as the laser beam was not broken or tracking was not lost the tracker would provide the three dimensional coordinates of the target at a maximum sampling frequency of $450 \mathrm{~Hz}$. Throughout the tests the calibration bar was fixed and the laser tracker was not moved. To reinitialize, therefore, it was only necessary to provide the laser tracker controller with the previously-determined radial distance and check that the azimuth and elevation angles were the same as those measured during the first initialization.

The accuracy of the operation of the laser tracker was checked from time to time with the following tests:

1. Lock the beam on the target and record the azimuth and elevation angles. Without moving the target rotate the head of the laser tracker by 180 degrees (rotation about the azimuth axis) and the reflecting mirror by 180 degrees (rotation about the elevation axis) lock on the target and record the azimuth and elevation angles. The difference in the values of the angles before and after the rotation should be 180 degrees plus or minus an error.

2. Lock the beam on the target, switch to spherical coordinates and observe the fluctuations in the values of the radial distance, the azimuth and elevation angles. As long as there are no significant air flow velocity and temperature changes and the target remains fixed the fluctuations should be random and no systematic drift should be observed.

3. Initialize the laser tracker and then move the calibration bar to a new location. Move the target from one of the bar target mounting locations to the other and record the coordinates of the two locations. Calculate the distance between the two mounting locations and compare it to the previously measured distance, during its manufacturing, with high precision metrology instruments.

4. Initialize the laser tracker and record the coordinates of one of the two bar target mounting locations. Move the target away from that location and then return and put it back in the same location. Compare the coordinates measured before and after the move. 
Due to small amplitude oscillations of most robot arms, even under steady state static conditions, the laser tracker is usually programmed to sample several times the position of the target and then average to obtain the coordinates for a single position observation. An experiment was performed and it was found that the robot used for the tests had no measurable amplitude oscillations, under steady state static conditions, at the simulated location of the sensor nest. It was then decided not to average the target position samples for all the tests reported here. This has the advantage of reducing the duration of the tests and of revealing the effects of any motion overshoots or undershoots.

The metrology tests described here were performed on April 10-May 14, 1990. The power to the laser was turned on and kept on for the entire duration of the tests. The robot was warmed up by running an exercise program for at least an hour prior to test execution. The only payload attached to the arm for all of the tests was the laser tracker target and its mounting bracket. The ambient temperature was 22-24 degrees $\mathrm{C}$, and relative humidity was in the range of $45-55 \%$.

Note: In the following sections, the coordinates $\mathrm{X}, \mathrm{Y}$, and $\mathrm{Z}$ referred to in the analysis of the data refer to laser tracker coordinates, unless otherwise noted.

\subsubsection{Teach Mode Control Tests}

The teach programming control mode is the predominant robot arm programming mode used today. It involves moving the robot arm to the desired locations, manually, through a teach pendant, or the keyboard. Once the robot arm is at a desired location the joint angles corresponding to that location are recorded. In the majority of the cases when the program is played back the robot arm is commanded to go back to the prerecorded joint angles, although sometimes it might be more convenient to use the calculated Cartesian coordinates, which correspond to those joint angles.

The main part of the FTS performance test will consist of teach mode control moves, in which the robot arm will be commanded to move from one or more initial positions to one or more previously-taught sensor nest locations. The objective of the tests was to measure the accuracy and repeatability errors of a robot arm when it is trying to reach the simulated FTS sensor nest location under teach mode control from the simulated initial positions. It 
was also desired to investigate the error variation as a function of the number of the test cycles, as well as the variation and drift of the achieved position.

The taught position used for the teach mode tests was S2. The eight RIA initial positions are used so that the robot moves through a large portion of the useful workspace during the test. The overall procedure of the tests is as follows:

Move to initial position

Move to $S 2_{\text {app }}$

Move to S2

Record position data

Move to $S 2_{\text {app }}$

Repeat above sequence for each different initial position

The completion of the above sequence for all initial positions constitutes performance of one test cycle. The duration of the $S 2_{\text {app }}$-to-S2 motions was 15 seconds, resulting in an approximate average Cartesian velocity of $30 \mathrm{~mm} / \mathrm{s}(1.2 \mathrm{in} / \mathrm{s})$. This is quite slow, although it is probably representative of how fast the arm will move to the sensor nest position on DTF-1. Each repetition of this sequence takes about $6 \mathrm{~min}$.

The Static Position (PTP motion) Accuracy, "is a statistical measure of the spatial deviation between commanded and achieved robot positions", [RIA 90]. The testing and calibration workstation calculates and prints the accuracy errors as defined by both [RIA 90] and [ISO 90]. The formulas used are the following:

$$
\begin{aligned}
& \mathrm{dPA}=\frac{1}{\mathrm{~N}} \sum_{\mathrm{i}=1}^{\mathrm{N}} \mathrm{d}_{\mathrm{i}} \cdot(4.2) \\
& \mathrm{d}_{\mathrm{i}}=\sqrt{\left(\mathrm{x}_{\mathrm{a} 1}-\mathrm{x}_{\partial}\right)^{2}+\left(\mathrm{y}_{\mathrm{a}-1}-\mathrm{y}_{\partial}\right)^{2}+\left(\mathrm{z}_{\mathrm{a} 1}-\mathrm{z}_{\partial}\right)^{2}} .
\end{aligned}
$$

$$
S P A=\sqrt{\frac{\sum_{i=1}^{N}\left(d_{i}-d P A\right)^{2}}{N-1}} .
$$


DeltaL $=\sqrt{\left(\overline{\mathrm{x}}-\mathrm{x}_{\partial}\right)^{2}+(\overline{\mathrm{y}}-\mathrm{y})^{2}+(\overline{\mathrm{z}}-\mathrm{z})^{2}}$.

$\operatorname{DeltaL}_{\mathrm{x}}=\overline{\mathrm{x}}-\mathrm{x}_{\mathrm{c}}$.

$\operatorname{DeltaL}_{\mathrm{y}}=\overline{\mathrm{y}}-\mathrm{y}_{\mathrm{c}}$.

$\operatorname{DeltaL}_{\mathrm{z}}=\overline{\mathrm{z}}-\mathrm{z}_{\mathrm{c}}$

$\bar{x}=\frac{1}{N} \sum_{i=1}^{N} x_{a i} ; \bar{y}=\frac{1}{N} \sum_{i=1}^{N} y_{a i} ; \bar{z}=\frac{1}{N} \sum_{i=1}^{N} z_{a i}$

Where:

$\mathrm{dPA}$, is the Positional Accuracy as defined by RIA, except that in this case only one measurement (commanded) position was used, the simulated sensor nest location.

$\mathrm{N}$, is the number of measurement test cycles used.

$\mathrm{d}_{\mathrm{i}}$, is the magnitude of the accuracy error deviation at the ith measurement.

$\mathrm{x}_{\mathrm{ai}}, \mathrm{y}_{\mathrm{ai}}, \mathrm{z}_{\mathrm{ai}}$, are the coordinates of the ith measured (achieved) position.

$\mathrm{x}_{\mathrm{c}}, \mathrm{y}_{\mathrm{c}}, \mathrm{z}_{\mathrm{c}}$, are the coordinates of the commanded position, in this case the simulated sensor nest location.

SPA, is the standard deviation of dPA.

DeltaL, is the Unidirectional Positioning Accuracy as defined by ISO, except that in this case only one measurement (commanded) position was used, the simulated sensor nest location. It should be called unidirectional because the final approach to the commanded position is always from the same direction.

$\bar{x}, \bar{y}, \bar{z}$, are the coordinates of the mean of the $\mathrm{N}$ measured (achieved) positions.

The Positional Repeatability, "is the measure of deviations between achieved robot positions and the mean of those positions after ordering the robot to the same pose $\mathrm{N}$ times", [RIA 90]. The testing and calibration workstation calculates and prints the accuracy errors as defined by both [RIA 90] and [ISO 90]. The formulas used are the following:

$\mathrm{rREP}=\frac{1}{\mathrm{~N}} \sum_{\mathrm{i}=1}^{\mathrm{N}} \mathrm{m}_{\mathrm{i}}$ 
$m_{i}=\sqrt{\left(x_{a i}-\bar{x}\right)^{2}+\left(y_{a i}-\bar{y}\right)^{2}+\left(z_{a i}-\bar{z}\right)^{2}}$

$S R E P=\sqrt{\frac{\sum_{i=1}^{N}\left(m_{i}-r R E P\right)^{2}}{N-1}}$.

$r=r R E P+3$ SREP

Where:

IREP, is the Repeatability as defined by RIA, except that in this case only one measurement (commanded) position was used, the simulated sensor nest location.

$\mathrm{m}_{\mathrm{i}}$, is the magnitude of the deviation at the ith measurement from the mean of the $\mathrm{N}$ measured (achieved) positions.

SREP, is the standard deviation of rREP.

$r$, is the Unidirectional Repeatability as defined by ISO, except that in this case only one measurement (commanded) position was used, the simulated sensor nest location. It should be called unidirectional because the final approach to the commanded position is always from the same direction.

The orientation accuracy and repeatability errors of the interface plate of the robot arm could not be measured with the laser tracker available at the present time.

\subsubsection{1.a Teach mode joint angles kinematics control}

For this version of the accuracy and repeatability tests, joint interpolated motion was used to move to all positions. The RRC controller was used to obtain equivalent joint positions for $S 2, S 2_{\text {app }}$, and the initial positions. The test was repeated seven times, resulting in 56 recorded points. Before running the tests, the robot was moved to the nominal S2 position, and the robot joint angle positions and laser tracker readings for this position were recorded to use this as the taught point. The actual joint values which were recorded for this point were then used as command angles for $\mathrm{S} 2$ for executing the tests. 


\subsubsection{1.b Analysis and Conclusions}

The laser tracker coordinates of the 56 measured positions were divided into 7 analysis groups. The first group contained the coordinates of the first 8 measured positions, which correspond to the first 8 cycles ( 8 vertices of the RIA standard path) of the test. The second group contained the coordinates of the first 16 measured positions. The third group contained the coordinates of the first 24 measured positions, etc., so each subsequent group contained the coordinates of the previous group plus the coordinates of the next 8 positions until all 56 were included. The data contained in each group were analyzed separately and the results of the analysis were used to determine the effect of the number of measured positions on the results.

Table 4.1 in the Appendix shows the results of the analysis of the last group of data which contains all 56 measured positions. First, the laser tracker measured coordinates of the commanded, previously taught, position are printed. The dimension of the coordinates are in $\mathrm{mm}$ as are all the dimensions in all the tables and plots reported here. Then the coordinates of the laser tracker-measured achieved positions are printed. Finally the ISO and RIA defined accuracies and repeatabilities are calculated and printed.

Figure 4.4 is a three dimensional plot of the measured achieved positions (triangular marks), their mean position (cross mark), and the commanded position (square mark). The coordinate frame in that figure is that of the laser tracker after it was translated to the centroid of those positions.

Figure 4.5 is a plot of the same positions as they are projected on a plane defined by the $\mathrm{X}$ and $\mathrm{Z}$ coordinate axes. As can be seen the cluster of points forms a "galactic cloud" with an orientation which is approximately orthogonal to the orientation of the axis of the first joint of the robot arm. Because of that, it is suspected, although it has not been verified, that positioning errors from the first joint drive are mostly responsible for the measured repeatability errors. To reach the simulated sensor nest position the arm has to extend itself significantly thus making it sensitive to angular errors from the first joint drive. The points also seem to be oriented in neat rows and columns. This is because their distances are very small and they have been positioned at the resolution-limited positions of the laser tracker instrument. 


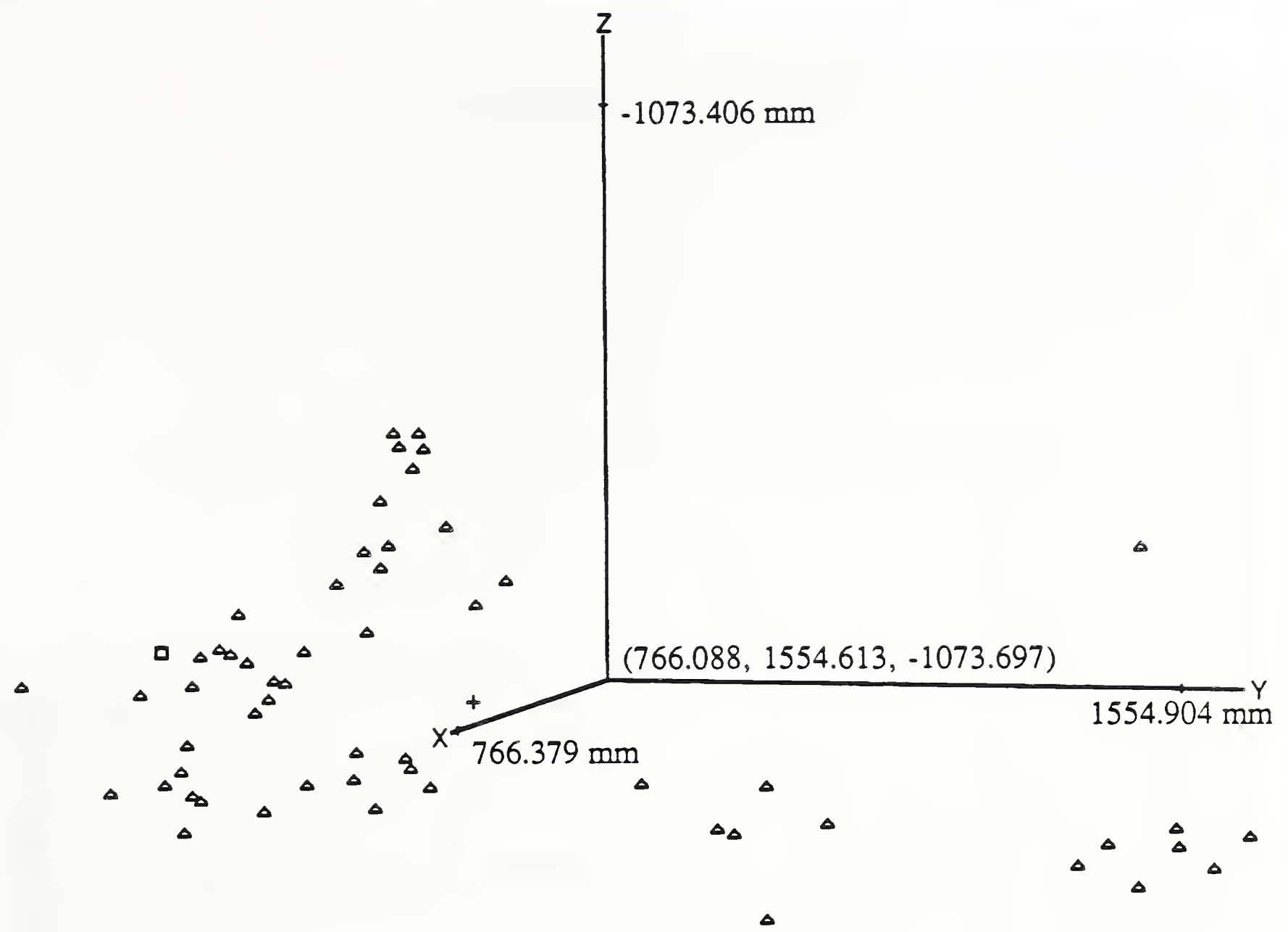

Plot of the PTP Test Achieved Positions

Each triangle is located at an achieved position

The square marks the commanded position

The cross marks the mean of the achieved positions

Figure 4.4 Teach mode joint angles kinematics control positions plot. 


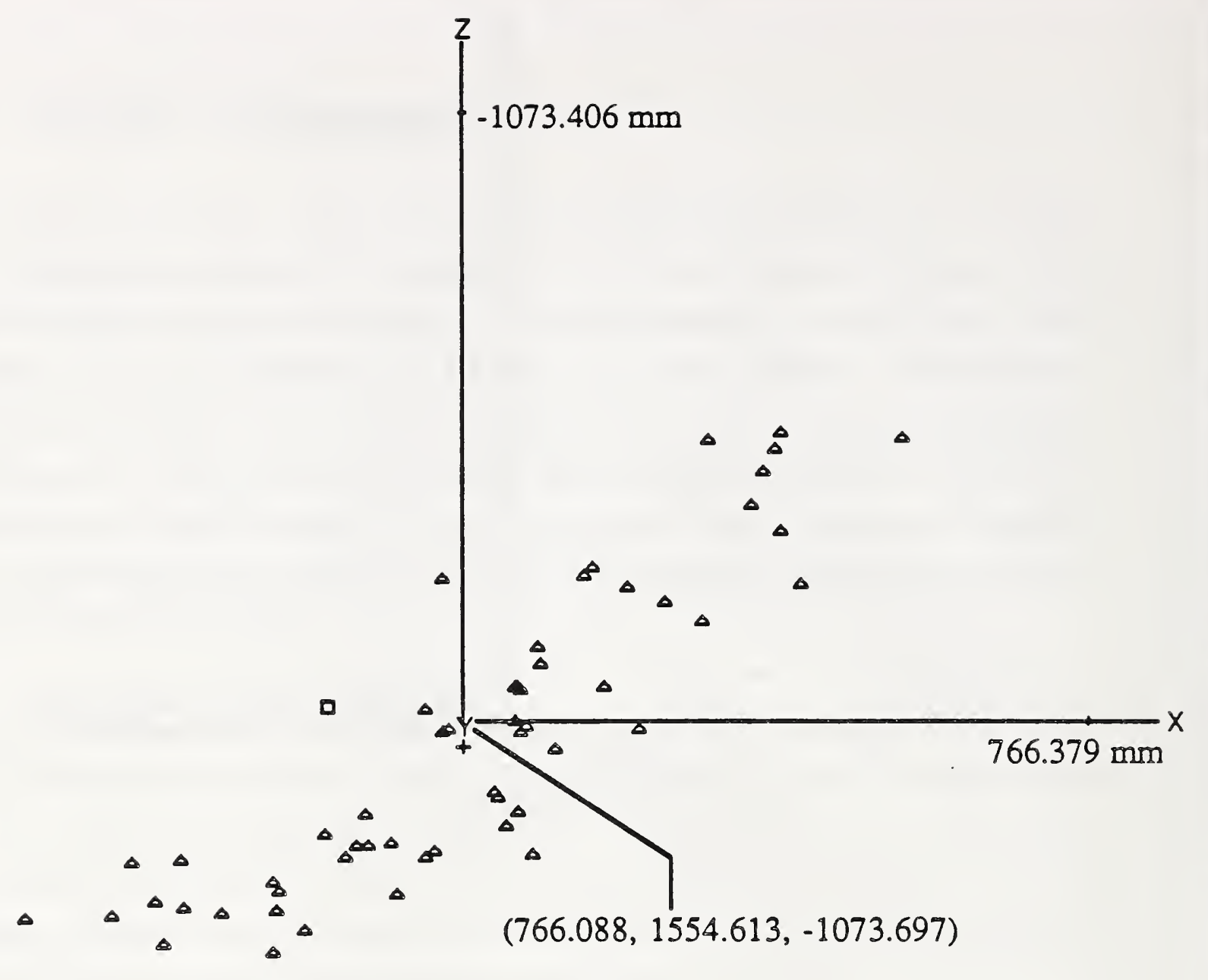

$\Delta$

Plot of the PTP Test Achieved Positions

Each triangle is located at an achieved position

The square marks the commanded position

The cross marks the mean of the achieved positions

Figure 4.5 Teach mode joint angles kinematics control positions plot. 


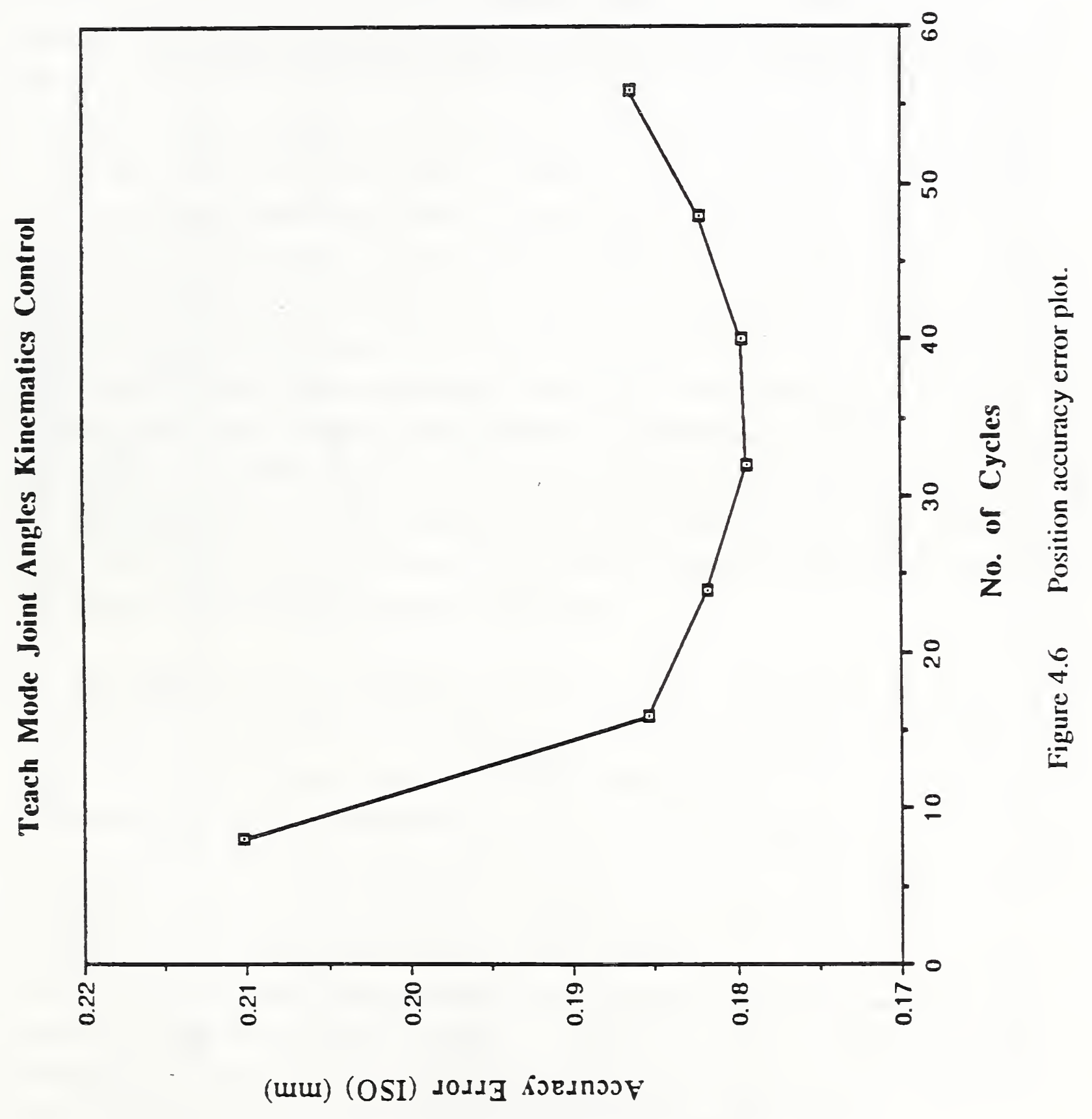

$18 \mathrm{c}$ 


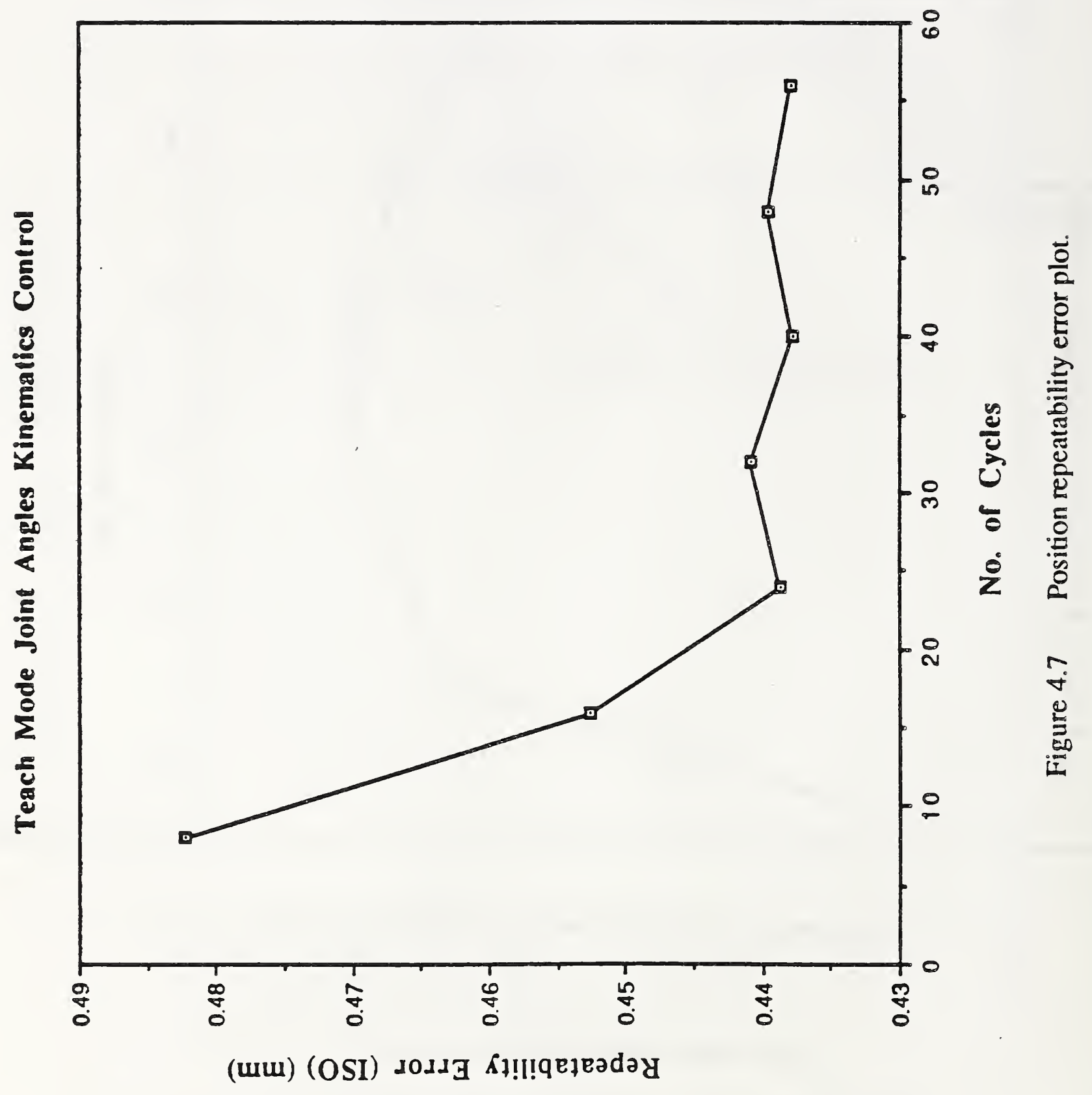


Figure 4.6 is a plot of the ISO defined accuracy error versus the number of cycles contained in each analysis group. Figure 4.7 is a plot of the ISO defined repeatability error versus the number of cycles contained in each analysis group. As can be seen from these plots the errors seem to follow an exponential decay curve reaching an asymptote after 24 to 32 cycles. The small rise in the accuracy error after 40 cycles is not considered significant although it needs to be investigated.

To better understand the nature of this exponential decay of the accuracy and repeatability errors the $\mathrm{X}, \mathrm{Y}$, and $\mathrm{Z}$ axes, laser tracker coordinates of the 56 measured positions were plotted as a function of the number of cycles and are shown in Figures 4.8, 4.9, 4.10 respectively. These plots show a periodicity with a fundamental frequency of 8 cycles and a few smaller amplitude higher frequency oscillations. There is an obvious drift during the first 8 cycles, which corresponds to the first group of analysis data, and a less pronounced drift during the next 8 cycles. After the first 16 cycles the coordinates seem to follow a relatively stable periodic oscillation with a peak-to-peak amplitude of approximately 0.3 $\mathrm{mm}$ for the $\mathrm{X}$-axis coordinates, $0.5 \mathrm{~mm}$ for the $\mathrm{Y}$-axis coordinates, and $0.23 \mathrm{~mm}$ for the $\mathrm{Z}$ axis coordinates. The 8 cycles periodicity is expected as a result of using the 8 vertices of the RIA standard path as different initial positions. The drift during the first 8 to 16 cycles probably comes from thermal drift (the robot arm was exercised for a reasonable amount of time and cycles before each test), and dynamic motion transients which include friction. The errors measured during the first 8 to 16 cycles are probably representative of those which occur during all intermittent robot operations.

The variation in the measured achieved position was previously characterized by the repeatability error. From Fig. 4.5 it appears that the asymptotic value of the ISO defined repeatability error is approximately $0.44 \mathrm{~mm}$, which is larger than the peak-to-peak amplitude of the $\mathrm{X}$ and $\mathrm{Z}$-axes steady state oscillation, but not of the one along the $\mathrm{Y}$-axis. This indicates that if a robot behaves like the one used for these tests the peak-to-peak amplitude of its steady state oscillation would be a more appropriate measure of the variation of its achieved position rather than the repeatability error. The ISO repeatability error measured after the first 8 cycles $(0.482 \mathrm{~mm})$ is closer to predicting the peak-to-peak amplitude of the achieved position oscillation. In the case of the RIA defined repeatability (given by eq. 4.10 ) the value of $3 \times$ standard deviation should be added to the repeatability error in order to come close to the true achieved position variation. 


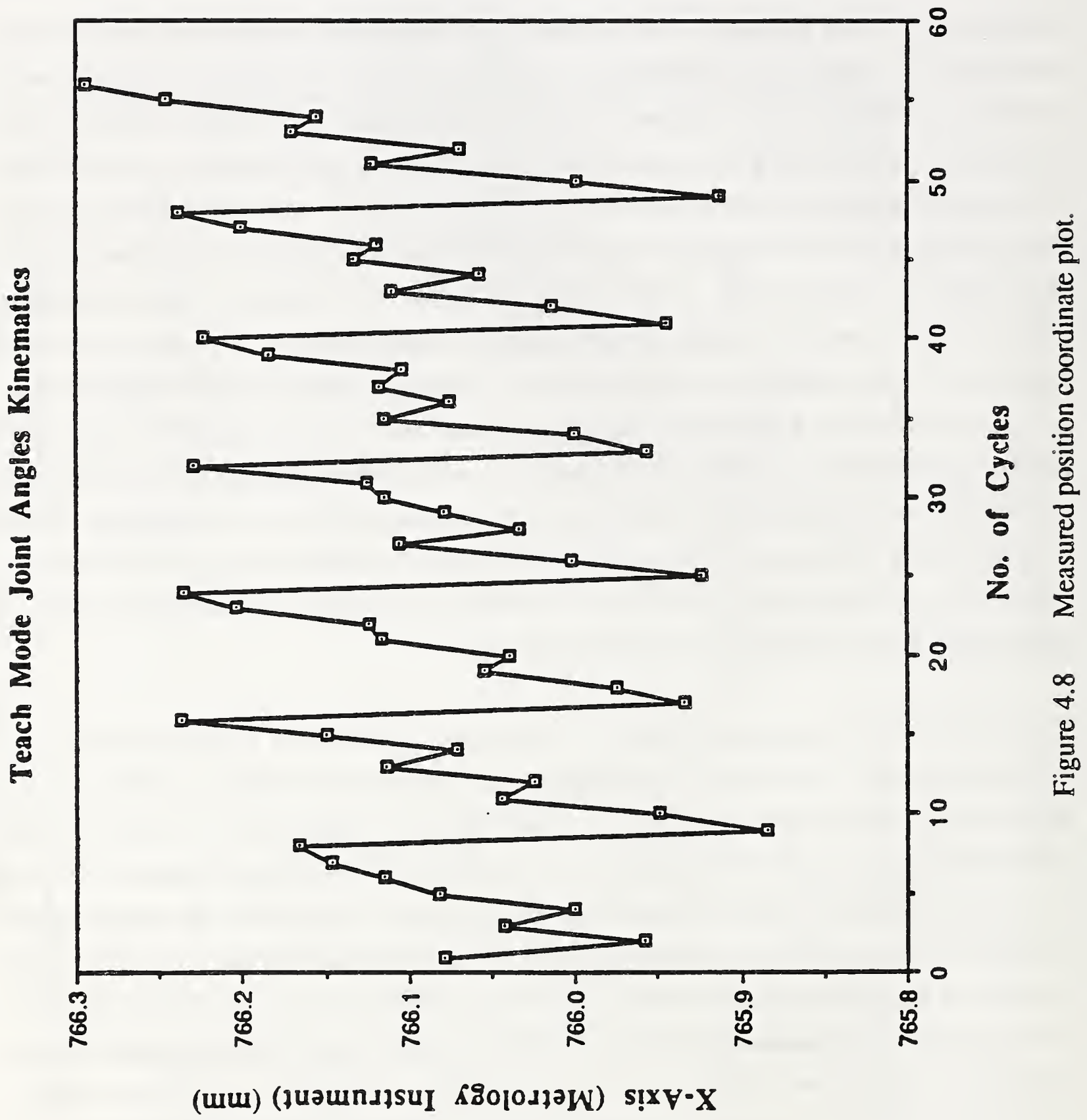




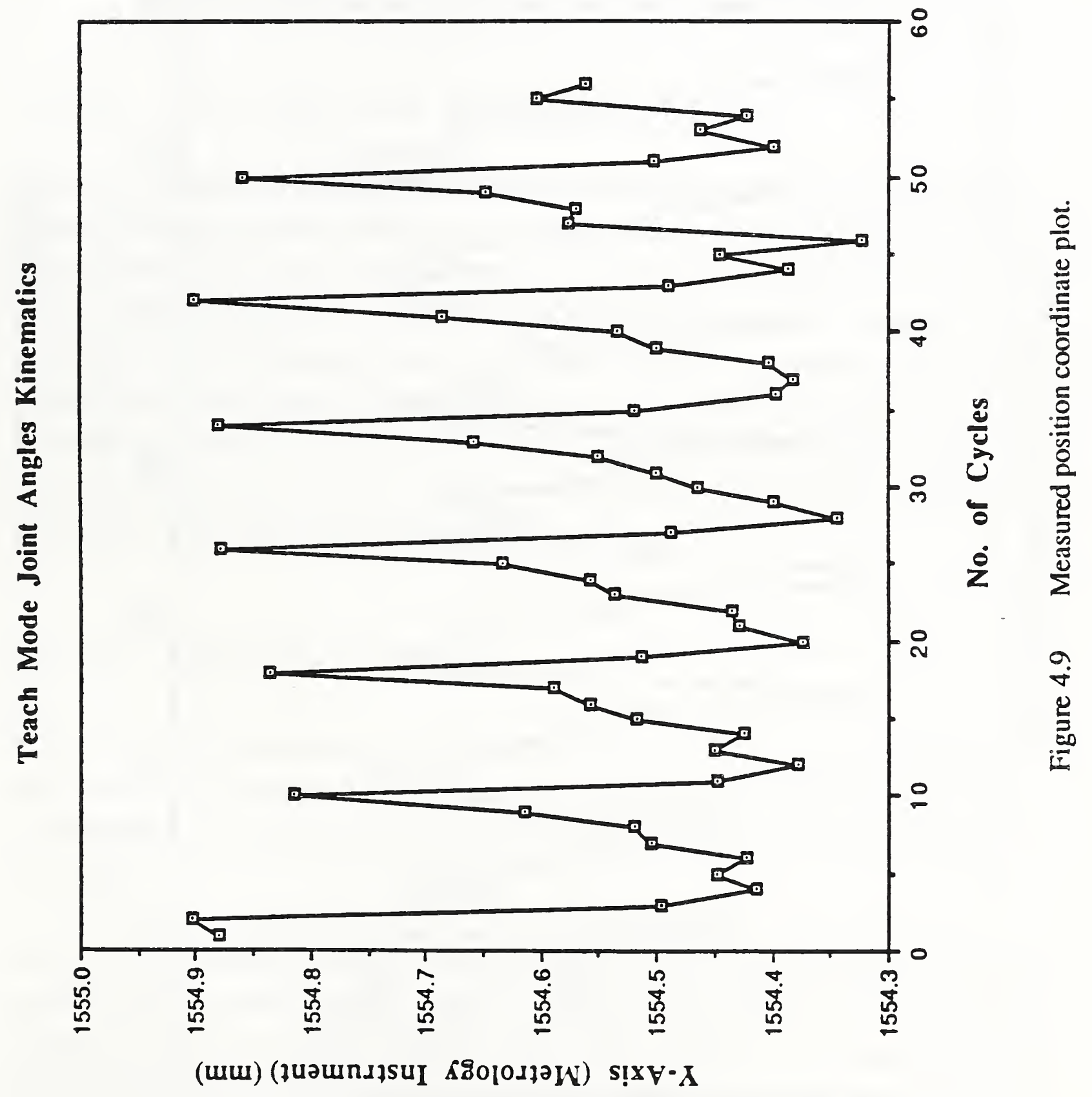




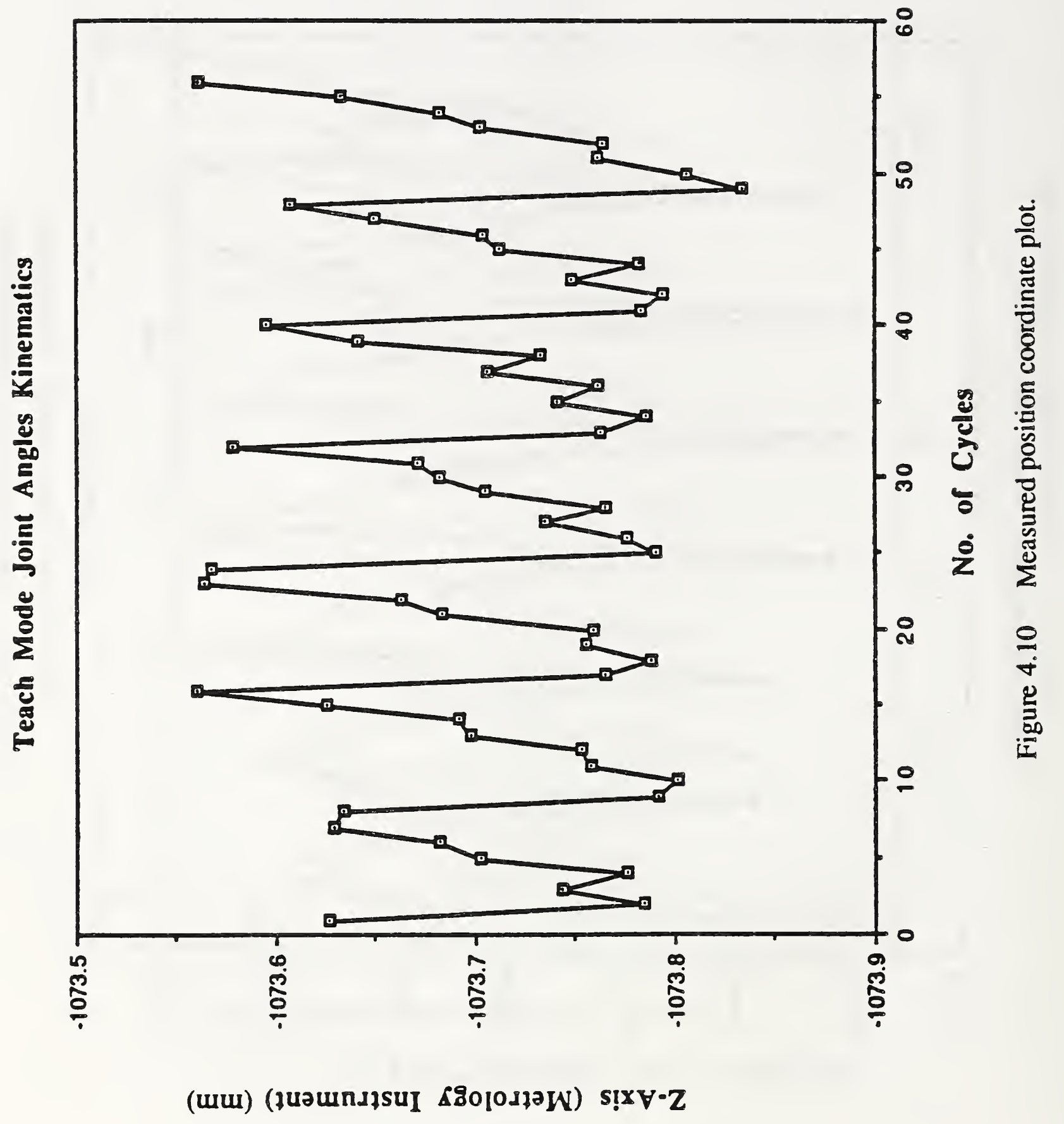


As can be seen from Figures 4.8, 4.9, 4.10 the measured achieved position is not really very random. For the same initial position of the RIA standard path approximately the same measured achieved position is obtained with a small amount of random displacement superimposed. Is that the result of the robot position control algorithm used, which does not stop servoing for as long as there is a joint angle position error? This of course raises the question, what would happen if only one initial position was used, would the random component dominate the achieved position? Another test which will be reported later will try to answer that question.

\subsubsection{2.a Teach mode inverse kinematics control}

This test is identical to the previous one, with the exception that $\mathrm{S} 2$ was recorded as a Cartesian position, and the Cartesian quintic polynomial trajectory algorithm was used to move between $S 2_{\text {app }}$ and S2 resulting in an approximately straight line motion. This motion would be necessary if the design of the sensor nest is such that a straight approach is required. The Cartesian quintic polynomial trajectory algorithm gives a better approximation of that type of trajectory than the joint interpolation algorithm. The inverse kinematics algorithm was therefore used for each trajectory point in these motions. The duration of the motions was the same as in the previous test, and joint interpolated motion was used to move between the initial positions and $S 2_{\text {app }}$.

\subsubsection{2.b Analysis and Conclusions}

The laser tracker coordinates of the 56 measured positions were again divided into 7 analysis groups. The data contained in each group were analyzed separately and the results of the analysis were used to determine the effect of the number of measured positions on the results.

Table 4.2 in the Appendix shows the results of the analysis of the last group of data which contains all 56 measured positions. Figure 4.11 is a three dimensional plot of the measured achieved positions (triangular marks), their mean position (cross mark), and the commanded position (square mark). The coordinate frame in that figure is that of the laser tracker after it was translated to the centroid of those positions. 


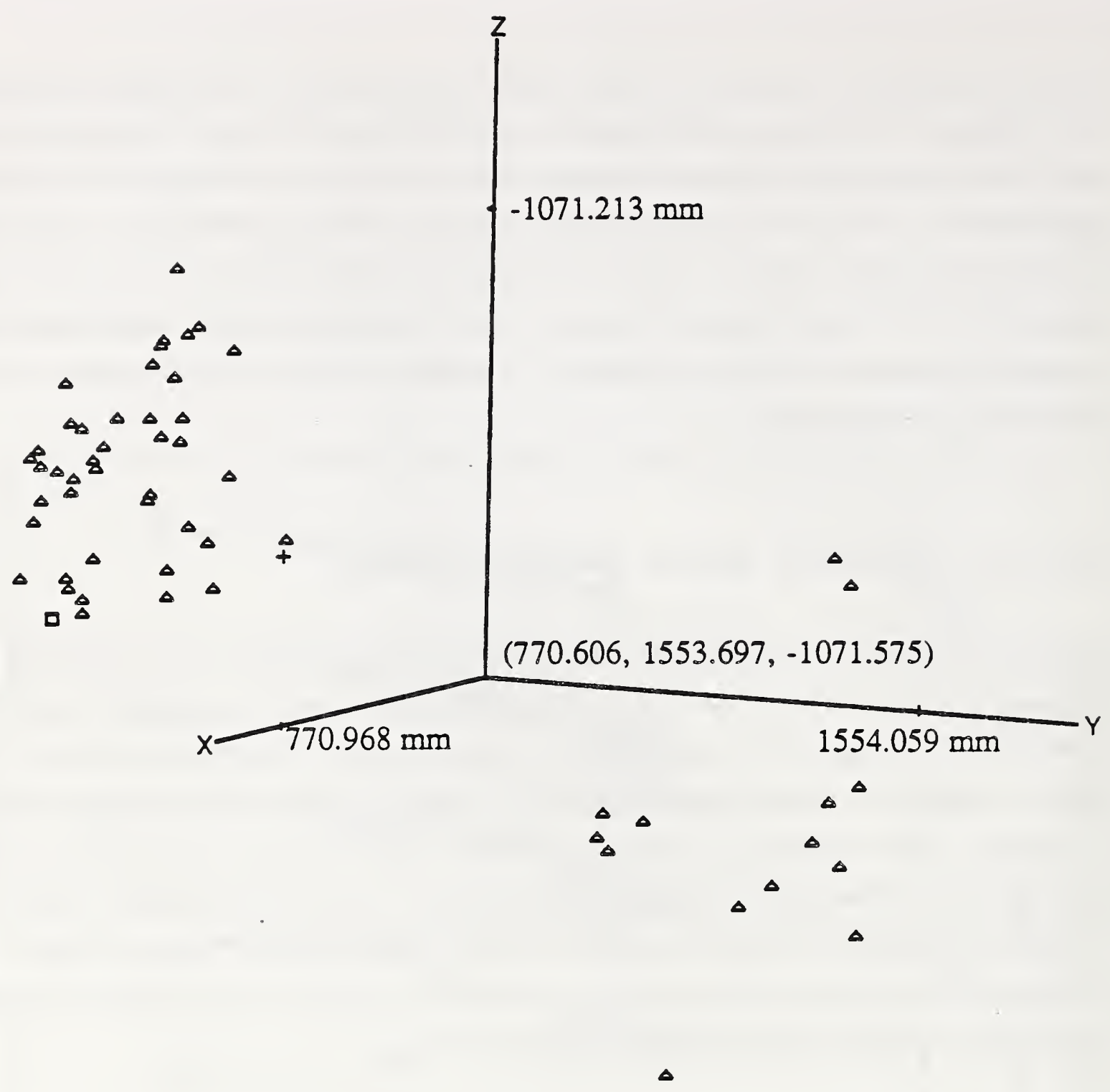

Plot of the PTP Test Achieved Positions

Each triangle is located at an achieved position

The square marks the commanded position

The cross marks the mean of the achieved positions

Figure 4.11 Teach mode inverse kinematics control positions plot. 


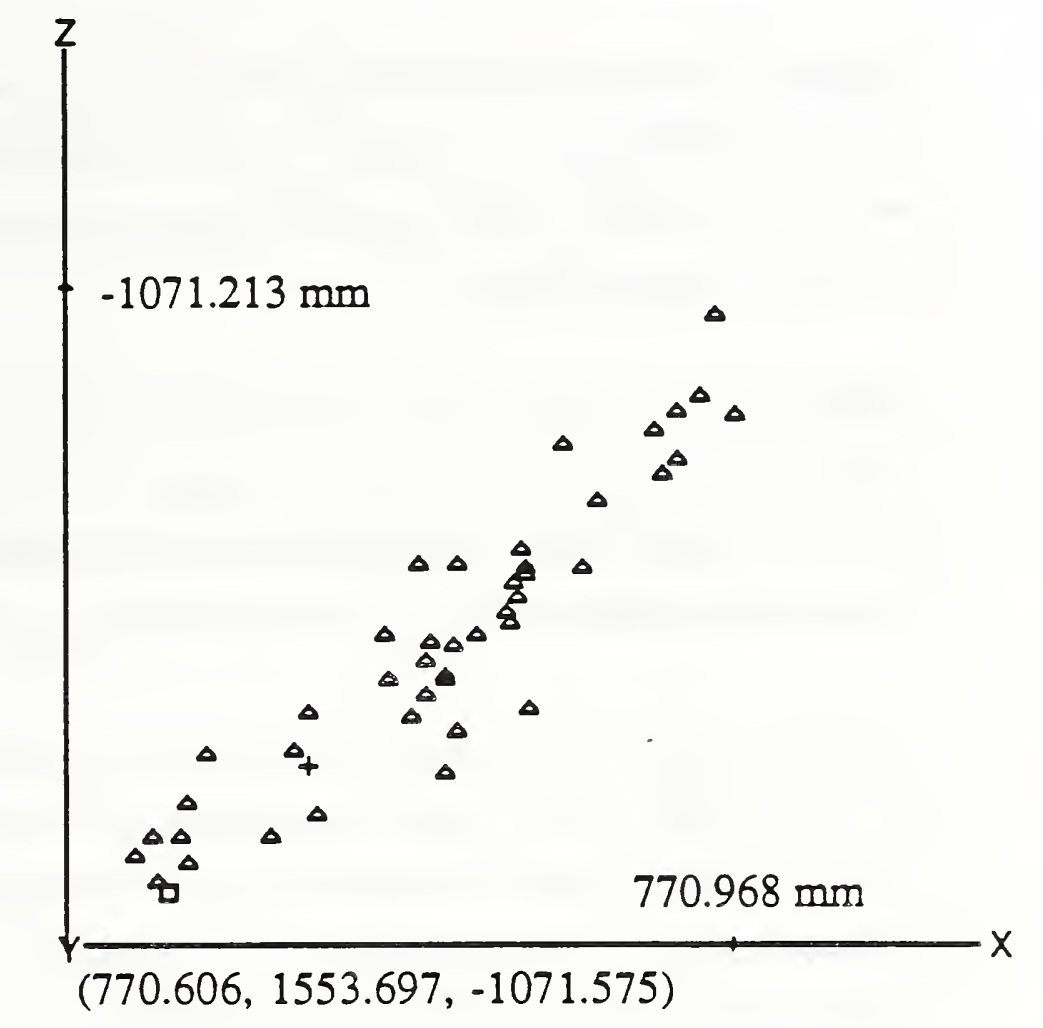

$\Delta$

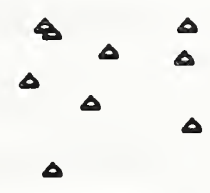

a

Plot of the PTP Test Achieved Positions

Each triangle is located at an achieved position

The square marks the commanded position

The cross marks the mean of the achieved positions

Figure 4.12 Teach mode inverse kinematics control positions plot. 
Figure 4.12 is a plot of the same positions as they are projected on a plane defined by the $\mathrm{X}$ and $\mathrm{Z}$ coordinate axes. As can be seen, the cluster of points again forms a galactic cloud with an orientation which is approximately orthogonal to the orientation of the axis of the first joint of the robot arm.

Figure 4.13 is a plot of the ISO defined accuracy error versus the number of cycles contained in each analysis group. Figure 4.14 is a plot of the ISO defined repeatability error versus the number of cycles contained in each analysis group. As can be seen from these plots the errors seem to decrease reaching an asymptote after 24 to 32 cycles.

To better understand the nature of this decrease of the accuracy and repeatability errors the $\mathrm{X}, \mathrm{Y}$, and $\mathrm{Z}$ axes, laser tracker coordinates of the 56 measured positions were plotted as a function of the number of cycles and are shown in Figures 4.15, 4.16, 4.17 respectively. These plots show again a periodicity with a fundamental frequency of 8 cycles and a few smaller amplitude higher frequency oscillations. There is an obvious drift during the first 8 cycles, which correspond to the first group of analysis data, and a less pronounced drift during the next 8 cycles. After the first 16 cycles the coordinates seem to follow a relatively stable periodic oscillation with a peak-to-peak amplitude of approximately 0.5 $\mathrm{mm}$ for the $\mathrm{X}$-axis coordinates, $0.55 \mathrm{~mm}$ for the $\mathrm{Y}$-axis coordinates, and $0.45 \mathrm{~mm}$ for the $\mathrm{Z}$-axis coordinates. The 8 cycles periodicity is expected as a result of using the 8 vertices of the RIA standard path as different initial positions. The drift during the first 8 to 16 cycles probably comes from thermal drift (the robot arm was exercised for a reasonable amount of time and cycles before each test), and dynamic motion transients which include friction. The errors measured during the first 8 to 16 cycles are probably representative of those which occur during all intermittent robot operations.

The variation in the measured achieved position was previously characterized by the repeatability error. From Fig. 4.14 it appears that the asymptotic value of the ISO defined repeatability error is approximately $0.67 \mathrm{~mm}$, which is larger than the peak-to-peak amplitude of the $\mathrm{X}, \mathrm{Y}$ and $\mathrm{Z}$-axes steady state oscillation. The ISO repeatability error measured after the first 8 cycles $(0.86 \mathrm{~mm})$ is large and reflects the large amplitude of the drift of the achieved position in the $\mathrm{Z}$ and $\mathrm{X}$-axes directions. In the case of the RIA defined repeatability (given by eq. 4.10 ) the value of $3 \times$ standard deviation should again be added to the repeatability error in order to come close to the true achieved position variation. 


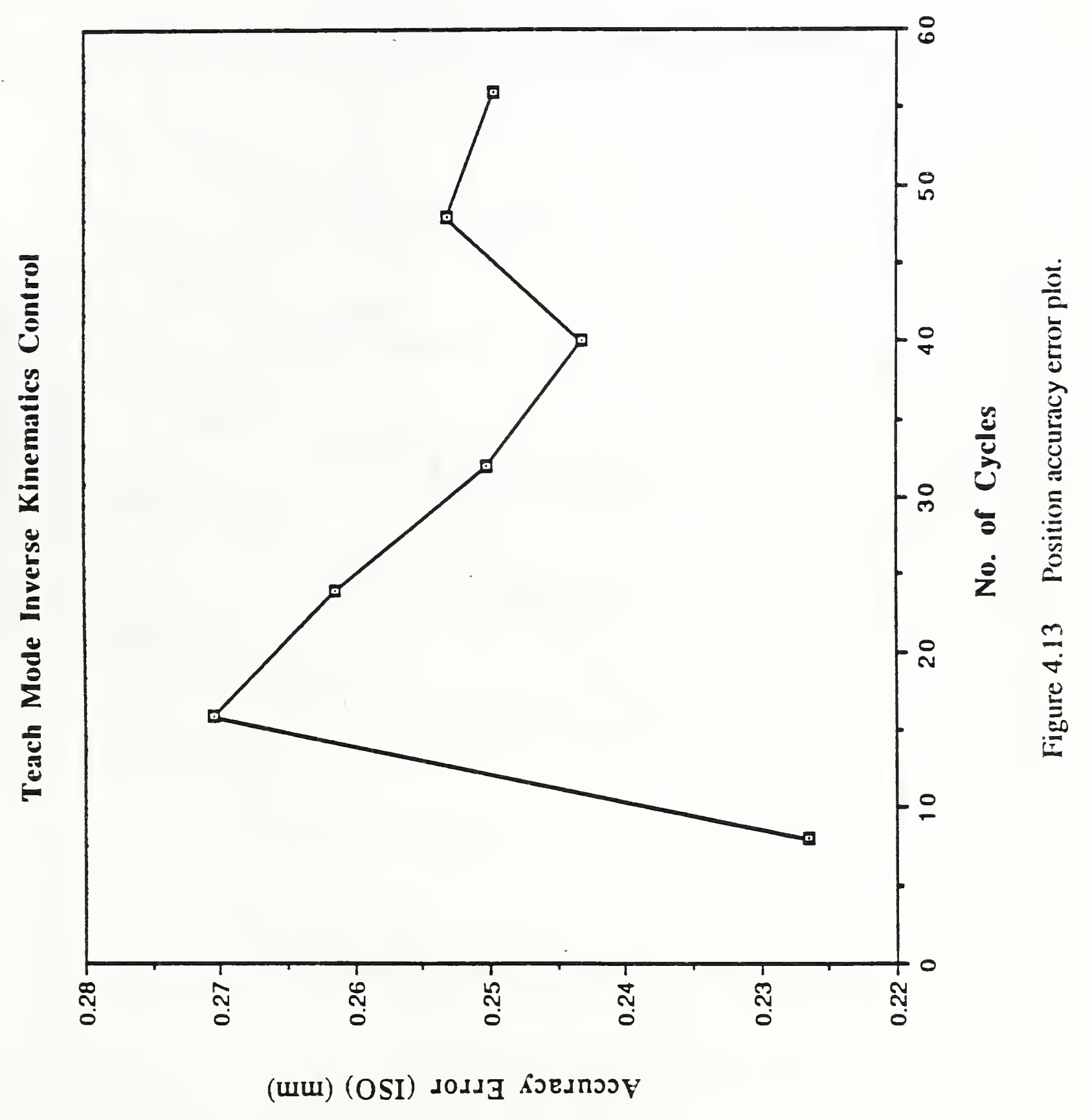




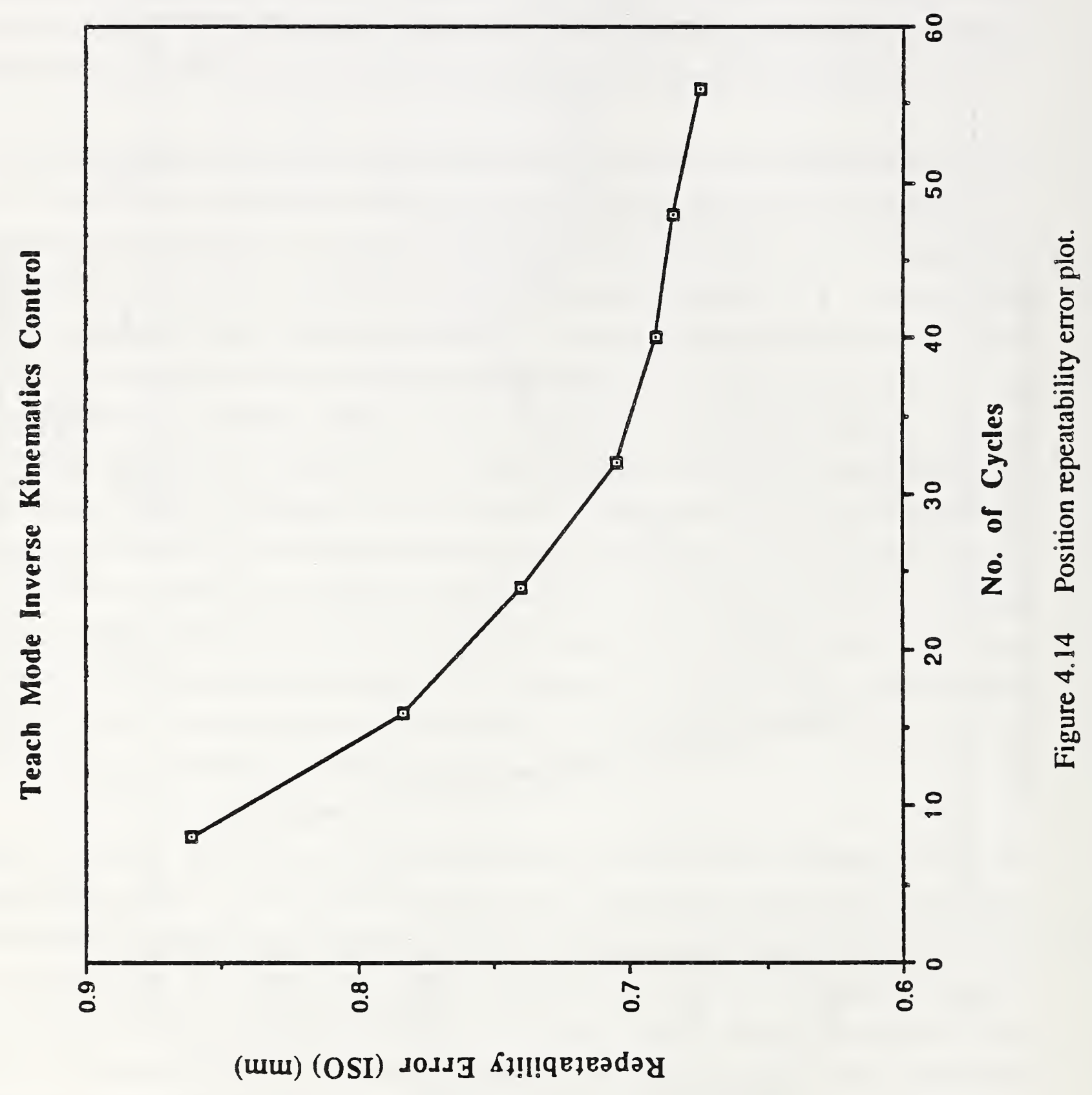




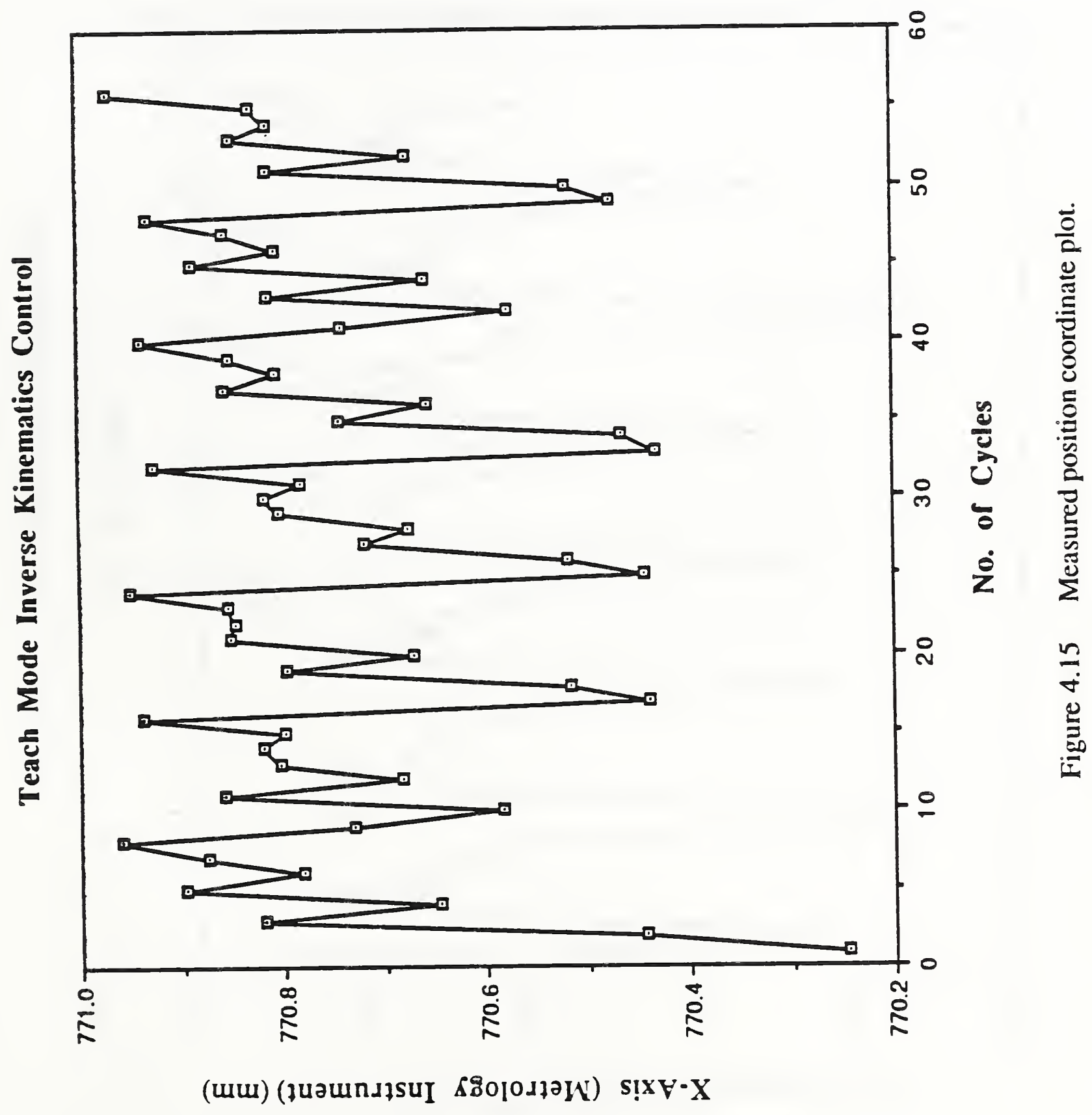




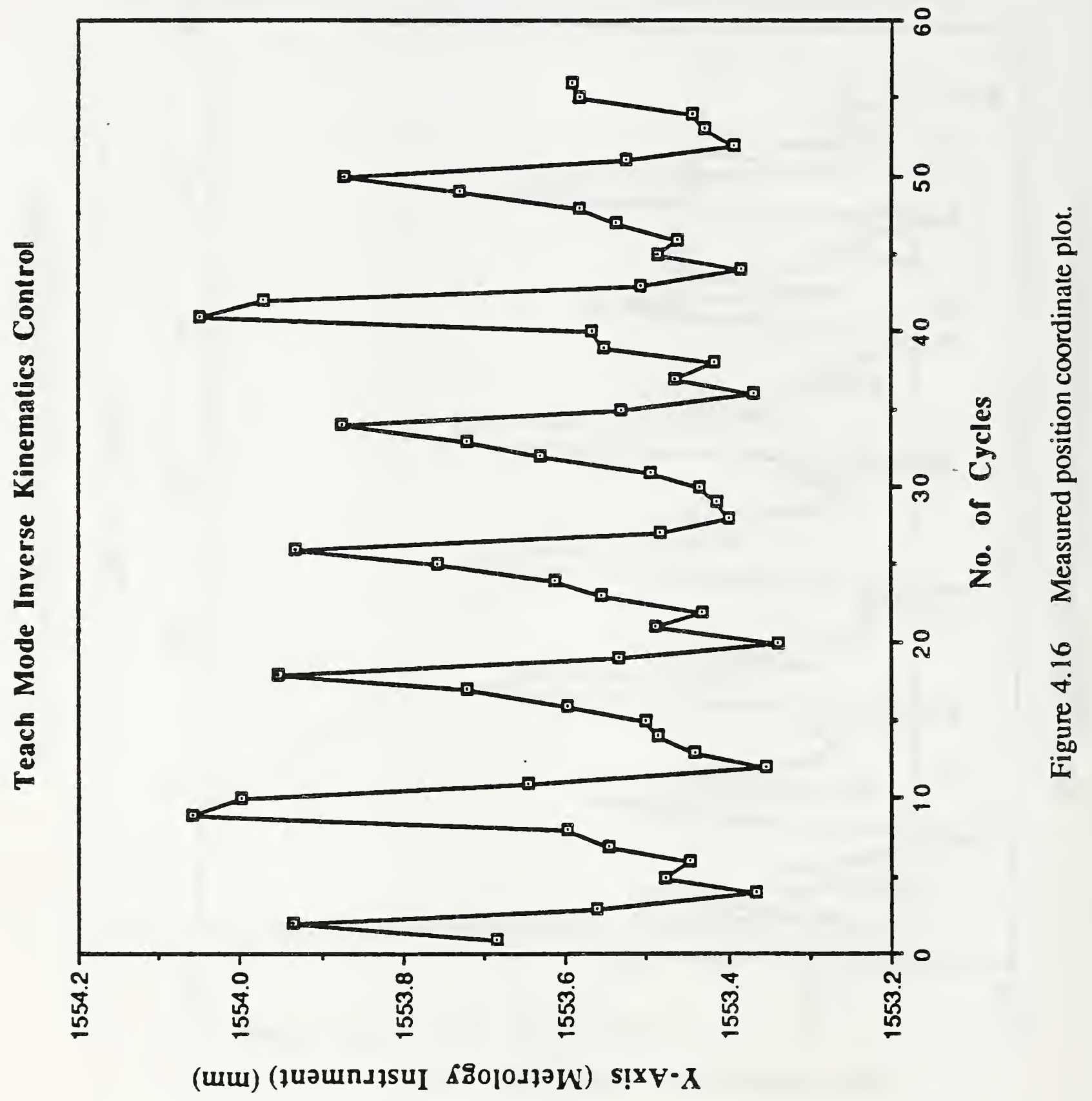




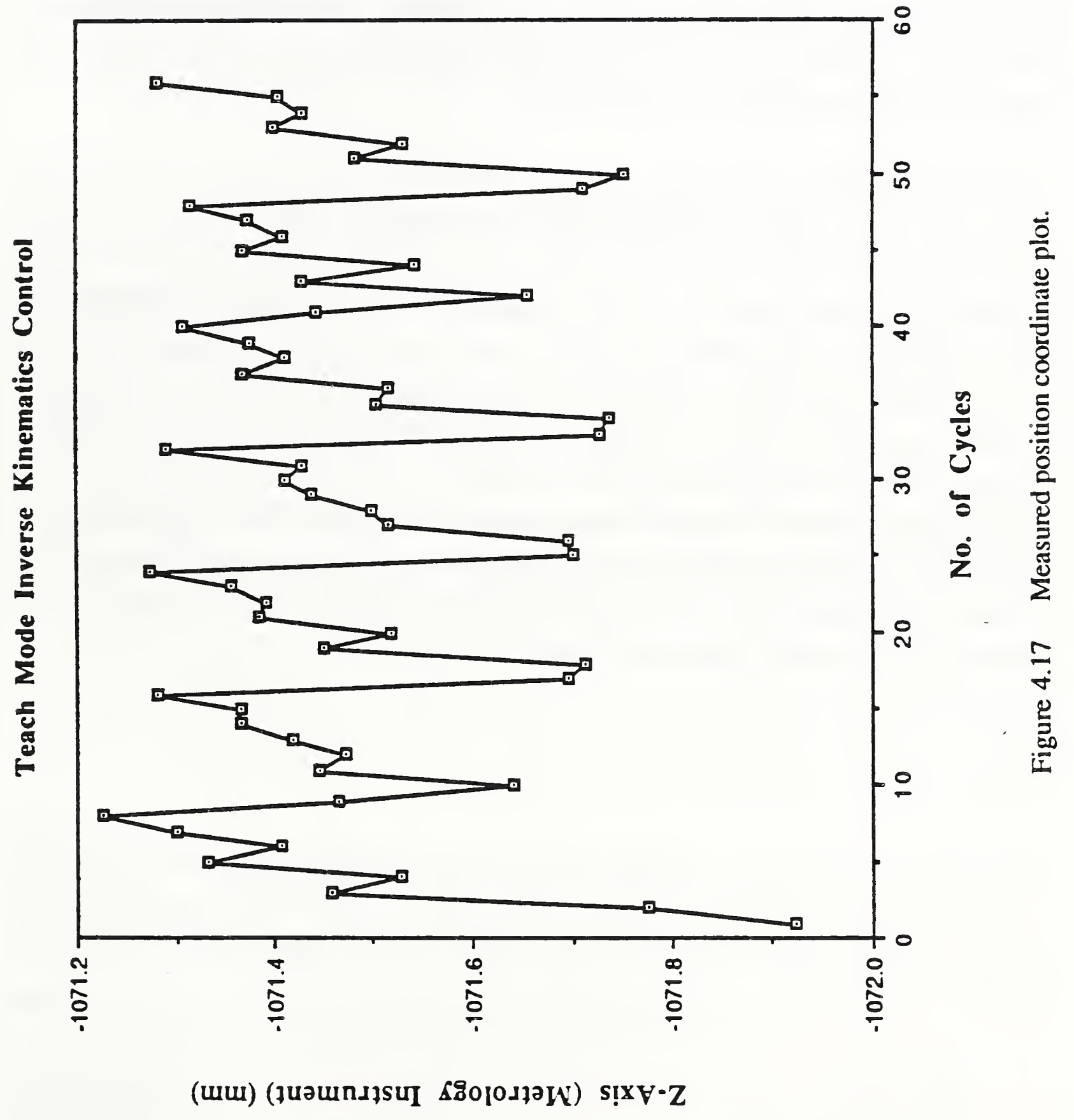


As can be seen from Figures 4.15, 4.16, 4.17 the measured achieved position is again not really very random. For the same initial position of the RIA standard path approximately the same measured achieved position is obtained with a small amount of random displacement superimposed.

Comparing the errors measured with joint interpolated motion with those obtained for Cartesian interpolated motion, it can be seen that in the second case they are larger for both accuracy and repeatability, and the peak-to-peak amplitudes of the coordinate oscillations. This makes sense since in the case of Cartesian interpolated motion the errors due to the inverse kinematics algorithms are also included.

\subsubsection{Coordinates Transformation Test}

The coordinates transformation is a mathematical relationship which relates the baseframe of the robot arm with the coordinate frame of the robot metrology instrument, for this work the laser tracker instrument. It allows the transformation of any metrology instrument measured coordinates to robot baseframe coordinates or the reverse, thus allowing all position and orientation information to be referred to a common frame of reference. The purpose of including the coordinates transformation determination was to support the offline programming test and the forward kinematics error analysis, another possible use is the non-destructive evaluation for the detection of any possible deformation of the FTS robotic arm and sensor nest, and their common foundation.

\subsection{2.a Test}

The purpose of this test is to determine the relative transformation between the base coordinate systems of the robot and the laser tracker. To make this determination, a number of points must be recorded in both robot and laser tracker coordinates. The procedure for this test is:

Move to S2

Record position data

Move to $\mathrm{S} 2+3 \mathrm{~mm}$ in the world $\mathrm{X}$ direction

Record position data 
Move to S2

Record position data

Move to $\mathrm{S} 2+3 \mathrm{~mm}$ in the world $\mathrm{Y}$ direction

Record position data

Move to S2

Record position data

Move to $\mathrm{S} 2+3 \mathrm{~mm}$ in world $\mathrm{Z}$ direction

Record position data

Move to S2

Record position data

The duration of each of the small motions was $3 \mathrm{~s}$. The time required for the entire sequence is about $0.5 \mathrm{~min}$. The position of the target was recorded by both the robot control system and by the laser tracker at the end of each motion. The sequence of motions was repeated twice, for a total of 14 data points.

\subsection{2.b Analysis and Conclusions}

The transformation is presented here as a translation vector and as a rotation matrix. If the translation vector is used to translate the robot base coordinate frame, its origin will coincide with the origin of the laser tracker coordinate frame. The rotation matrix consists of the directional cosines of the laser tracker coordinate frame axes unit vectors, with respect to the robot baseframe coordinate axes. If $x_{t}, y_{t}, z_{t}$, are the coordinates of the target with respect to the laser tracker coordinate frame and $x_{r}, y_{r}, z_{r}$, are the coordinates of the same target position with respect to the robot base coordinate frame then

$$
\left[\begin{array}{l}
x_{r} \\
y_{r} \\
z_{r}
\end{array}\right]=\left[\begin{array}{lll}
u_{x x} & u_{y x} & u_{z x} \\
u_{x y} & u_{y y} & u_{z y} \\
u_{x z} & u_{y z} & u_{z z}
\end{array}\right]\left[\begin{array}{l}
x_{t} \\
y_{t} \\
z_{t}
\end{array}\right]+\left[\begin{array}{l}
x_{0} \\
y_{0} \\
z_{0}
\end{array}\right] .
$$

where $\mathrm{x}_{0}, \mathrm{y}_{0}, \mathrm{z}_{0}$, are the coordinates of the translation vector, and

$$
\left[\begin{array}{lll}
u_{x x} & u_{y x} & u_{z x} \\
u_{x y} & u_{y y} & u_{z y} \\
u_{x z} & u_{y z} & u_{z z}
\end{array}\right]
$$


is the rotation matrix.

To determine the coordinates transformation the robot arm was moved to several positions inside the simulated sensor nest allowable workspace and the coordinates of the target were measured by the laser tracker and the robot controller. The best translation vector and rotation matrix to fit these data was determined by a double least squares optimization algorithm.

Table 4.3 in the Appendix gives the results of the coordinates transformation analysis for 14 target positions. First the coordinates of the target as measured by the laser tracker controller are given, then the coordinates of the same target positions as measured by the robot controller are given. Next, the coordinates of the target as measured by the laser tracker controller after they have been converted to robot control system coordinates using equation 4.14 are given. These coordinates should be very close to the robot control system measured coordinates printed above them; otherwise the transformation is not successful. Finally, the calculated transformation translation vector and rotation matrix are given. Figure 4.18 is a plot of the robot control system-measured target positions and the laser tracker controller-measured positions after they have been converted to robot control system coordinates. Ideally the corresponding points from those two sets should coincide with each other. In practice there will always be small differences due to numerical errors, errors in the kinematic models and motions of the robot and laser tracker controllers, and curve fitting errors of the transformation optimization algorithm.

Table 4.4 in the Appendix gives the results of the coordinates transformation analysis for the first 7 target positions out of the group of 14 used in the previous analysis and Figure 4.19 is the plot of those points. To evaluate the effectiveness of the transformation the differences between the robot controller-measured target positions and the laser tracker controller-measured coordinates of the same positions after they have been converted to robot controller coordinates was calculated and the root mean square error was evaluated. In the case of the group of the 14 data positions that was found to be $0.0544 \mathrm{~mm}$, while in the case of the group of the 7 data positions it was found to be $0.0585 \mathrm{~mm}$. Thus there is a slight improvement in the accuracy of the transformation when more data points are used. The difference is very small, though, which can be seen by examining the converted coordinates from the two tables. 


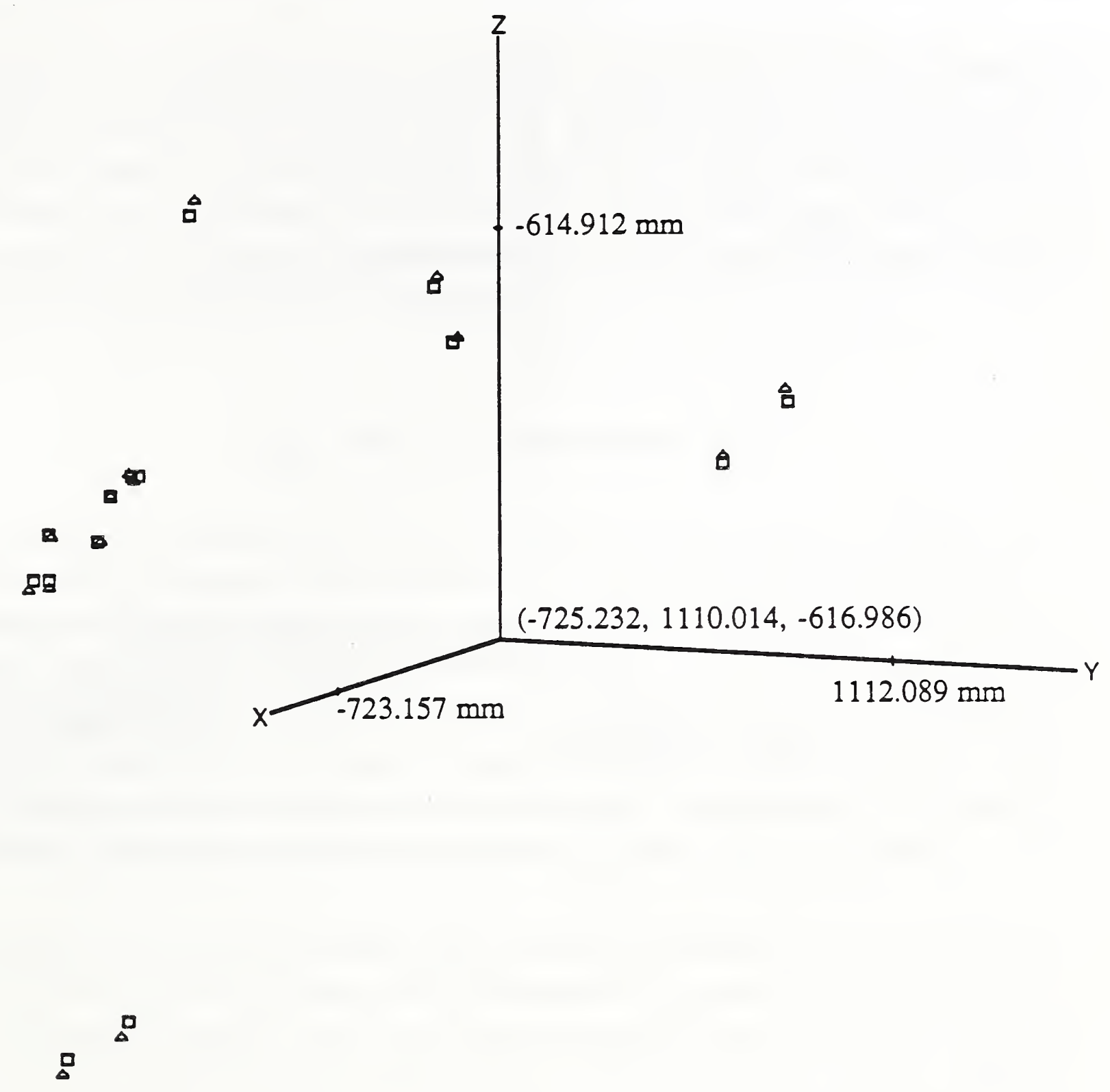

Each triangle is located at a robot controller measured position The square marks the robot metrology instrument measured positions which were converted to robot controller coordinates

Figure 4.18 Coordinates transformation analysis plot. 


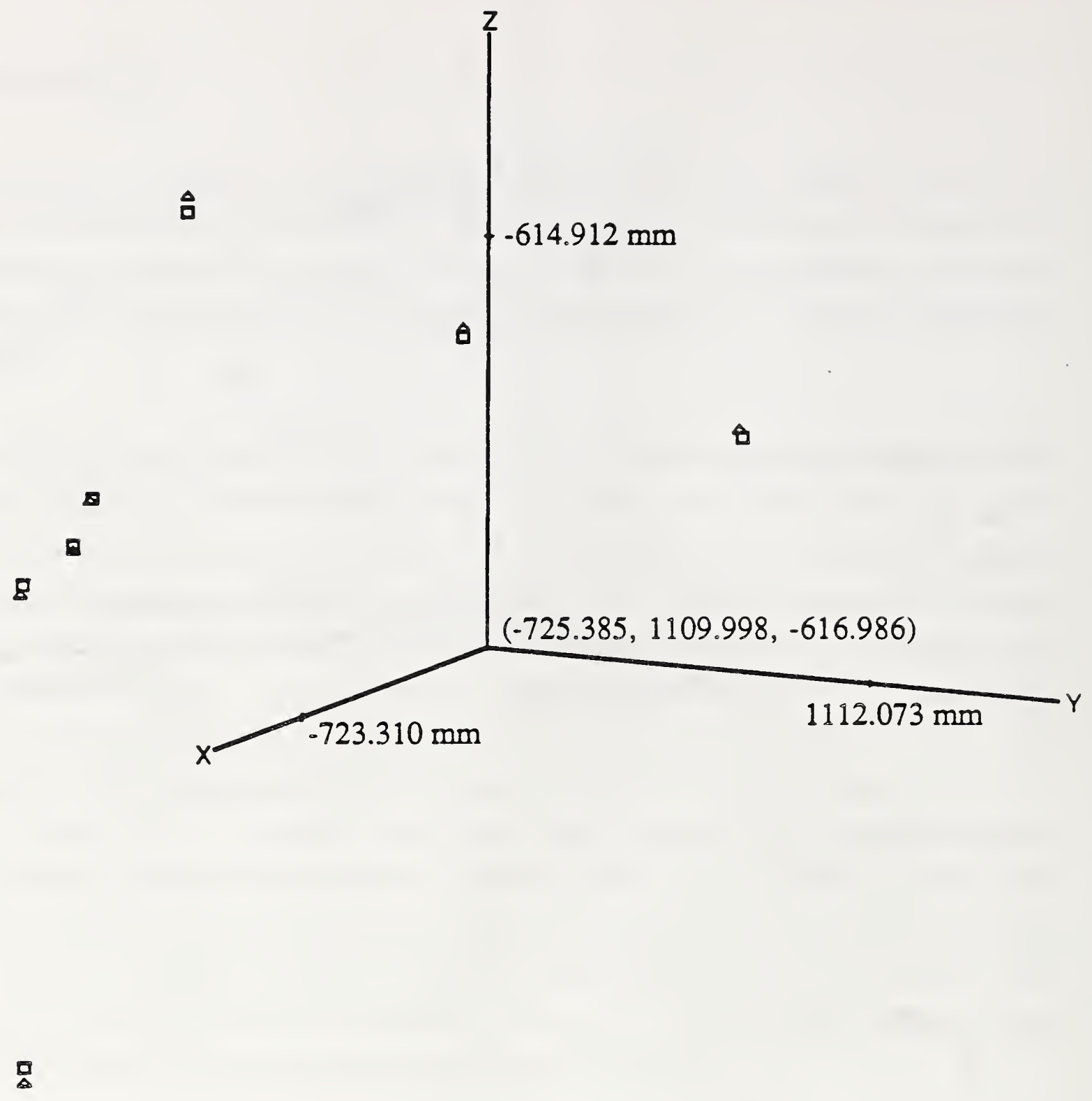

Each triangle is located at a robot controller measured position The square marks the robot metrology instrument measured positions which were converted to robot controller coordinates

Figure 4.19 Coordinates transformation analysis plot. 
To use the coordinates transformation test for non-destructive evaluation to detect any possible deformation of the FTS robotic arm and sensor nest, or their common foundation, the transformation vector would be compared with previous results to identify pronounced differences (a few millimeters or more). For this type of application a more thorough study of the effect of the number of data positions to errors in the calculation of the translation vector and rotation matrix should be performed.

\subsubsection{Off-Line Programming Tests}

In the off-line programming mode of operation, the robot position commands are generated by a computer and no teaching is involved during the generation of the commands. This of course requires the computer to have an accurate knowledge of the current robot arm model and its environment. This would be a preferable mode of operation for the FTS arm when it is working in the automatic mode, since it would be very difficult to teach it all the functions it is supposed to perform in space. Furthermore, since the dimensions of the arm and its surrounding objects might change due to heating, vibration or other reasons, teach control programming might not be very practical.

The objectives of the tests performed were to measure the accuracy and repeatability errors of a robot arm when it is trying to reach the simulated FTS sensor nest location under offline programming control. It was also desired to investigate the variation in the values of those errors as a function of the number of the test cycles and also the variation and drift of the achieved position. The simulated FTS sensor nest location was specified with respect to the laser tracker coordinate frame and then its coordinates were converted to robot controller Cartesian coordinates, which then became the new commanded position coordinates. The laser tracker coordinate frame coordinates were converted using the coordinates transformation determined by the group of the 14 data positions mentioned previously. In order to move to that commanded position the robot controller had to use the inverse kinematics algorithm to determine the corresponding joint angles.

Two different types of off-line programming tests were performed. In the first case the path of the arm was similar to that described in the "Teach Mode Control Tests" section and included the 8 positions of the RIA standard path. In the second case those positions were dropped and all motions initiated from the approach point and followed a straight line path motion to the commanded position. The reason this second path was used was to reduce 
the duration of the test, since this simple back and forth motion takes less time than the motion to the standard path positions. If the test results from these two cases are comparable then the second path would be preferable.

The testing and calibration workstation calculates and prints the accuracy and repeatability errors as defined by both [RIA 90] and [ISO 90]. The formulas used are those given by equations 4.2 to 4.13 .

\subsubsection{1.a Standard initial positions off-line programming}

The procedure for this test is very similar to the teach mode accuracy and repeatability test. The only difference is in how the goal position at S2 is determined. For the off-line programming test, instead of moving the robot to $S 2$ and using the recorded data as the goal position, the goal position is determined by transforming the position recorded by the laser tracker into robot coordinates. Since this position is a Cartesian position, the Cartesian trajectory algorithm and inverse kinematics algorithm are used to move between $S 2_{\text {app }}$ and $S 2$. The time required for this test is the same as for the teach mode tests (about 6 min per 8-position test).

\subsubsection{1.b Analysis and Conclusions}

The laser tracker coordinates of the 56 measured positions were again divided into 7 analysis groups. The data contained in each group were analyzed separately and the results of the analysis were used to determine the effect of the number of measured positions on the results.

Table 4.5 in the Appendix shows the results of the analysis of the last group of data which contains all 56 measured positions. Figure 4.20 is a three dimensional plot of the measured achieved positions (triangular marks), their mean position (cross mark), and the commanded position (square mark). The coordinate frame in that figure is that of the laser tracker after it was translated to the centroid of those positions.

Figure 4.21 is a plot of the same positions as they are projected on a plane defined by the $\mathrm{X}$ and $\mathrm{Z}$ coordinate axes. As with previous tests, the cluster of points forms a galactic cloud 


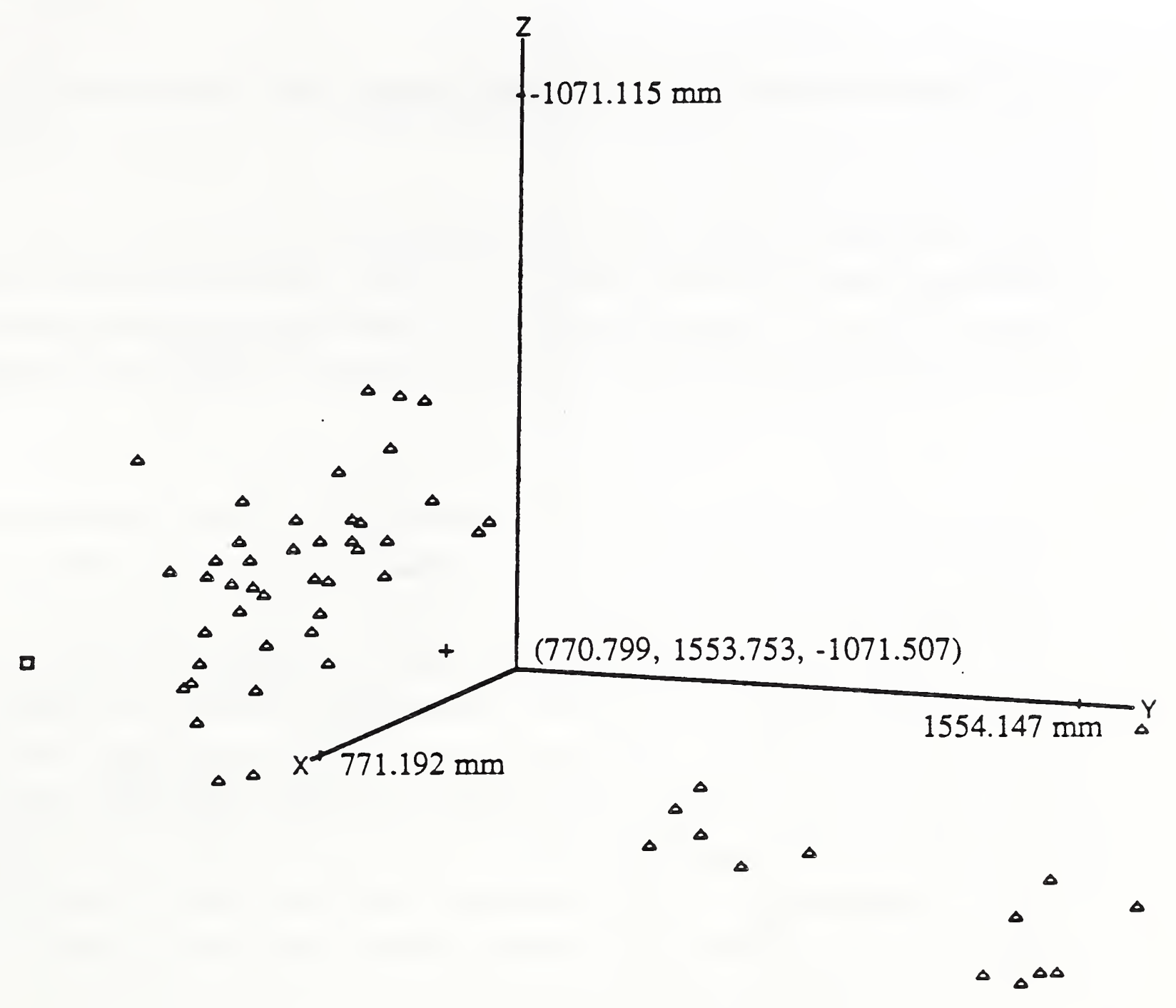

Plot of the PTP Test Achieved Positions

Each triangle is located at an achieved position

The square marks the commanded position

The cross marks the mean of the achieved positions

Figure 4.20 Standard initial positions off-line programming positions plot. 


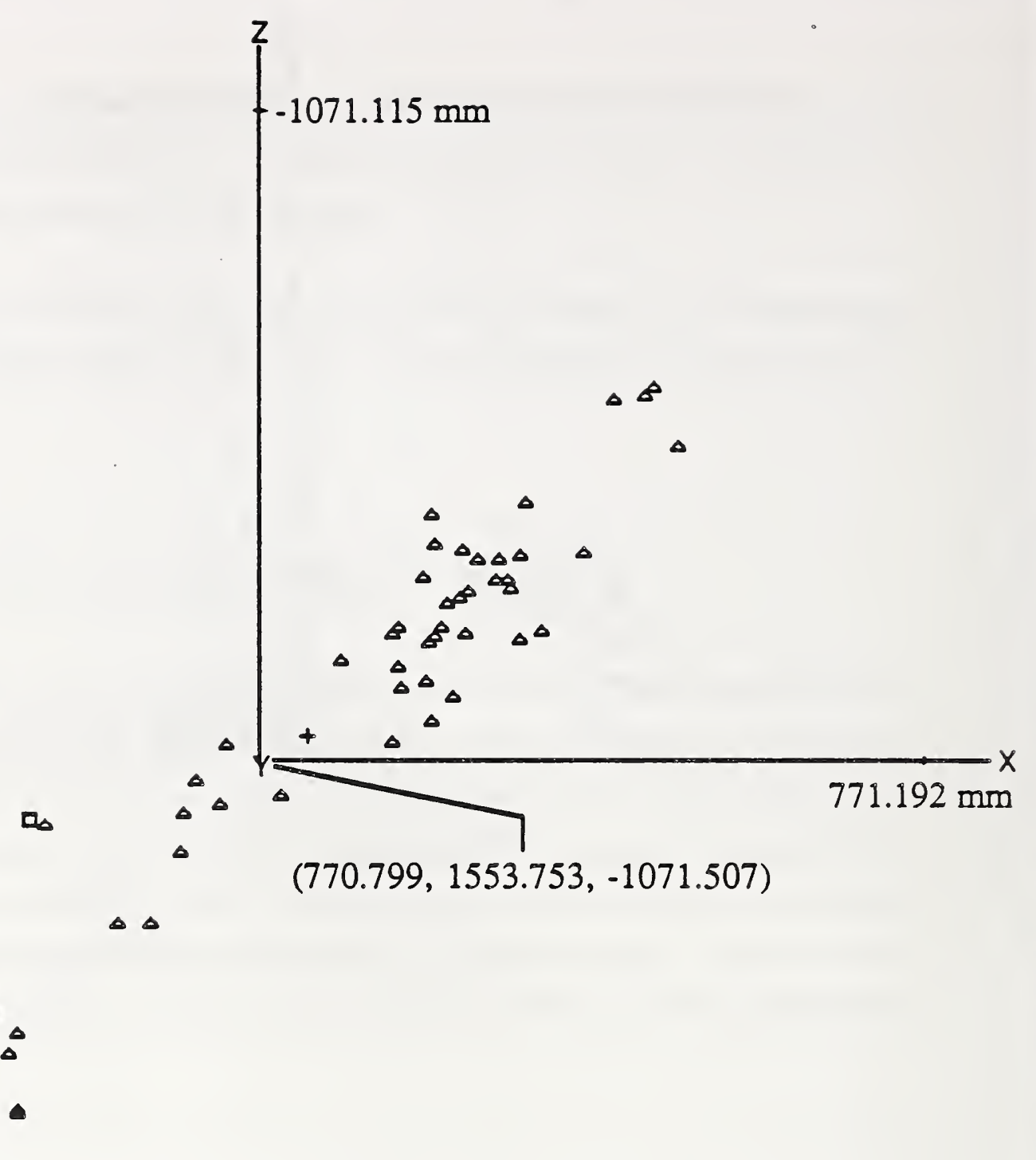

Plot of the PTP Test Achieved Positions

Each triangle is located at an achieved position

The square marks the commanded position

The cross marks the mean of the achieved positions

Figure 4.21 Standard initial positions off-line programming positions plot 
with an orientation which is approximately orthogonal to the orientation of the axis of the first joint of the robot arm.

Figure 4.22 is a plot of the ISO defined accuracy error versus the number of cycles contained in each analysis group. Figure 4.23 is a plot of the ISO defined repeatability error versus the number of cycles contained in each analysis group. As can be seen from these plots the errors seem to decrease exponentially reaching an asymptote after 24 to 32 cycles.

To better understand the nature of this decrease of the accuracy and repeatability errors the $X, Y$, and $Z$ axes, laser tracker coordinates of the 56 measured positions were plotted as a function of the number of cycles and are shown in Figures 4.24, 4.25, 4.26 respectively. These plots show again a periodicity with a fundamental frequency of 8 cycles and a few smaller amplitude higher frequency oscillations. There is an obvious drift during the first 8 cycles, which correspond to the first group of analysis data. After the first 8 cycles the coordinates seem to follow a relatively stable periodic oscillation with a peak-to-peak amplitude of approximately $0.45 \mathrm{~mm}$ for the $\mathrm{X}$-axis coordinates, $0.55 \mathrm{~mm}$ for the $\mathrm{Y}$-axis coordinates, and $0.43 \mathrm{~mm}$ for the $\mathrm{Z}$-axis coordinates. The 8 cycles periodicity is again expected, because of the different starting positions. The drift during the first 8 cycles probably comes from thermal drift (the robot arm was exercised for a reasonable amount of time and cycles before each test), and dynamic motion transients which include friction. Again, the errors during the first 8 to 16 cycles are probably characteristic of those which may be expected during intermittent operation.

The variation in the measured achieved position was previously characterized by the repeatability error. From Fig. 4.21 it appears that the asymptotic value of the ISO defined repeatability error is approximately $0.56 \mathrm{~mm}$, which is larger than the peak-to-peak amplitude of the $\mathrm{X}, \mathrm{Y}$ and $\mathrm{Z}$-axes steady state oscillation. The ISO repeatability error measured after the first 8 cycles $(0.664 \mathrm{~mm})$ is large and reflects the large amplitude of the drift of the achieved position in the Y-axis direction. In the case of the RIA defined repeatability (given by eq. 4.10 ) the value of $3 \times$ standard deviation should be added to the repeatability error in order to come close to the true achieved position variation.

As can be seen from Figures 4.24, 4.25, 4.26 the measured achieved position is not really very random. For the same initial position of the RIA standard path approximately the same measured achieved position is obtained with a small amount of random displacement 


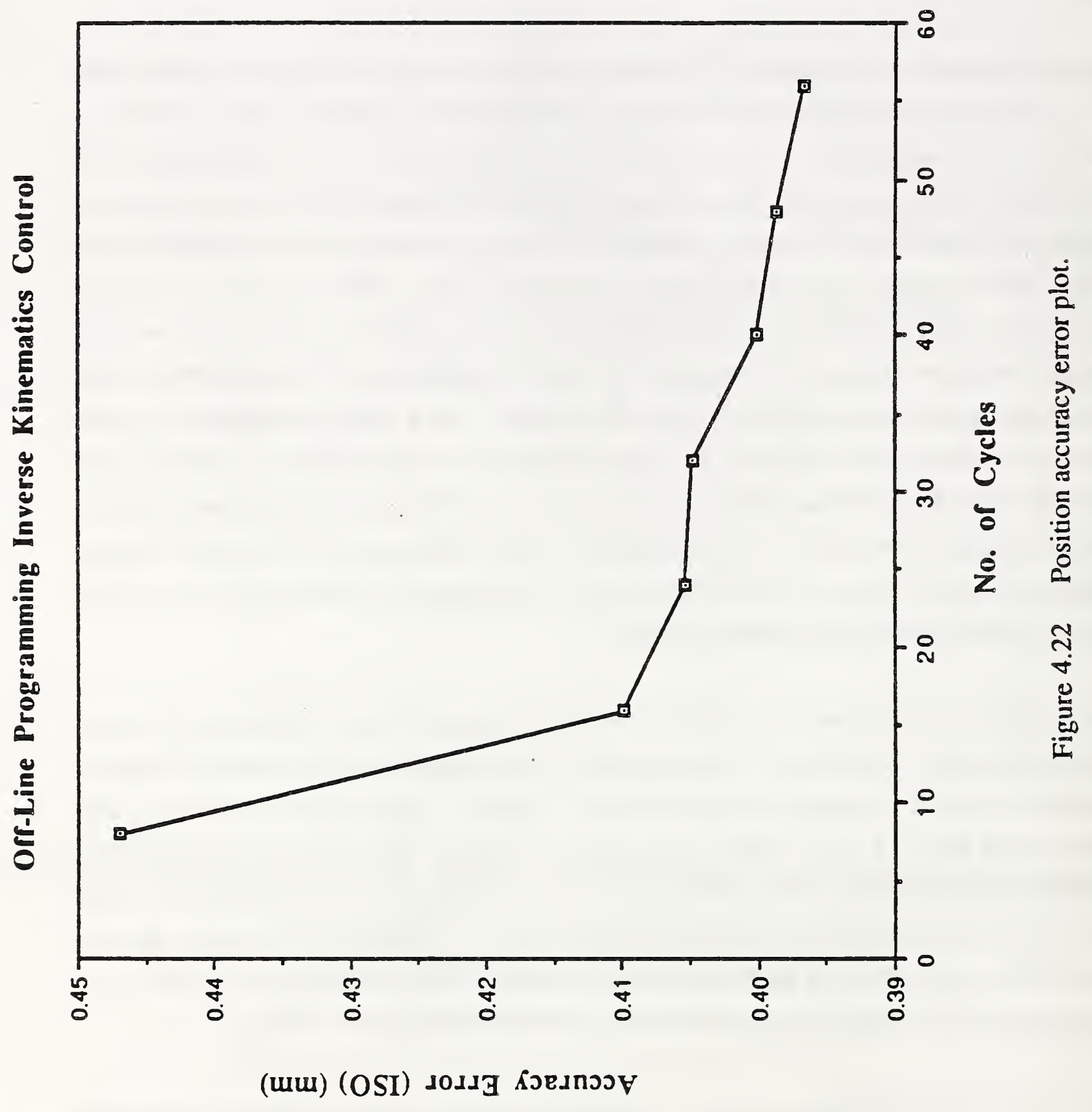




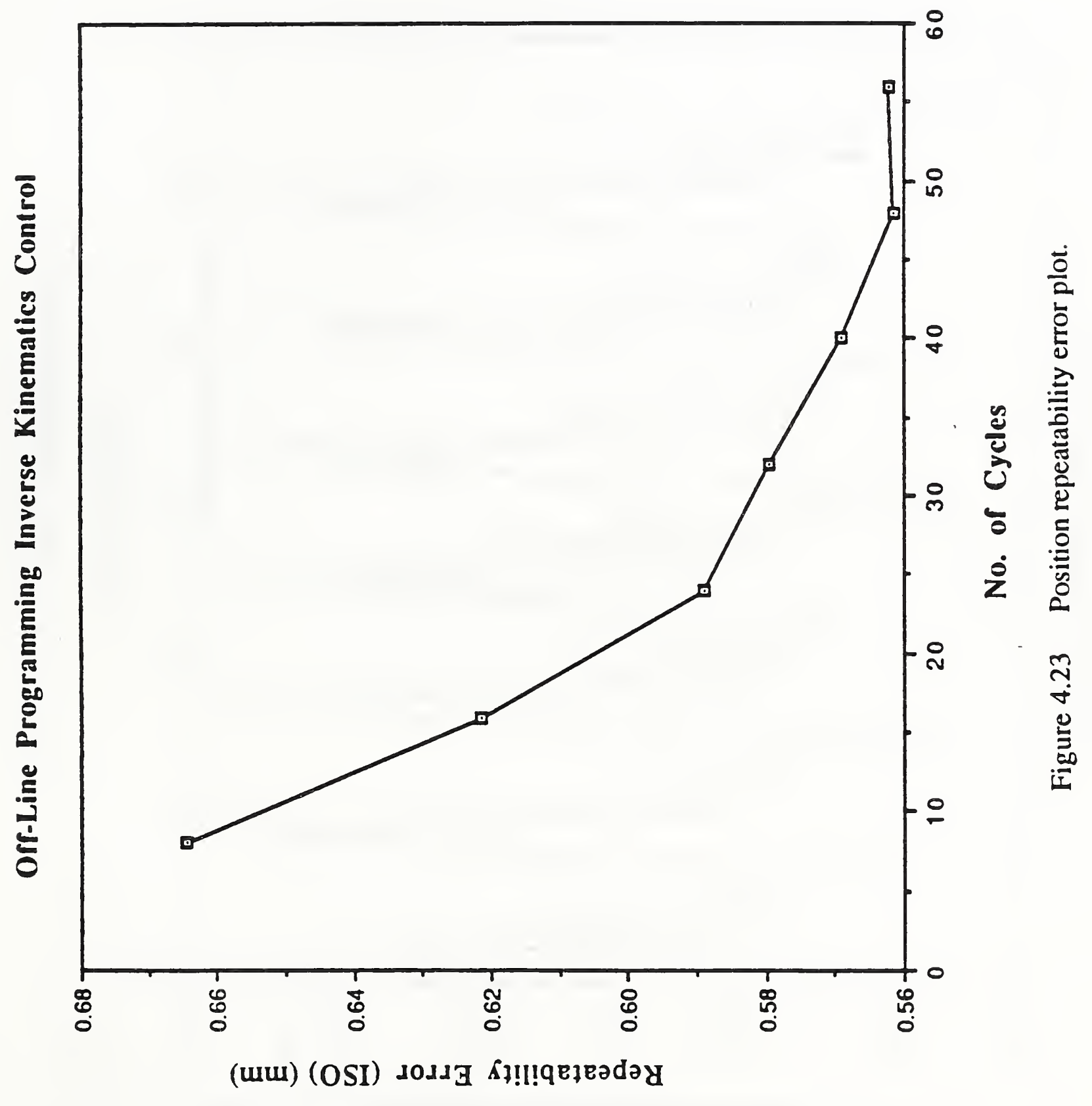




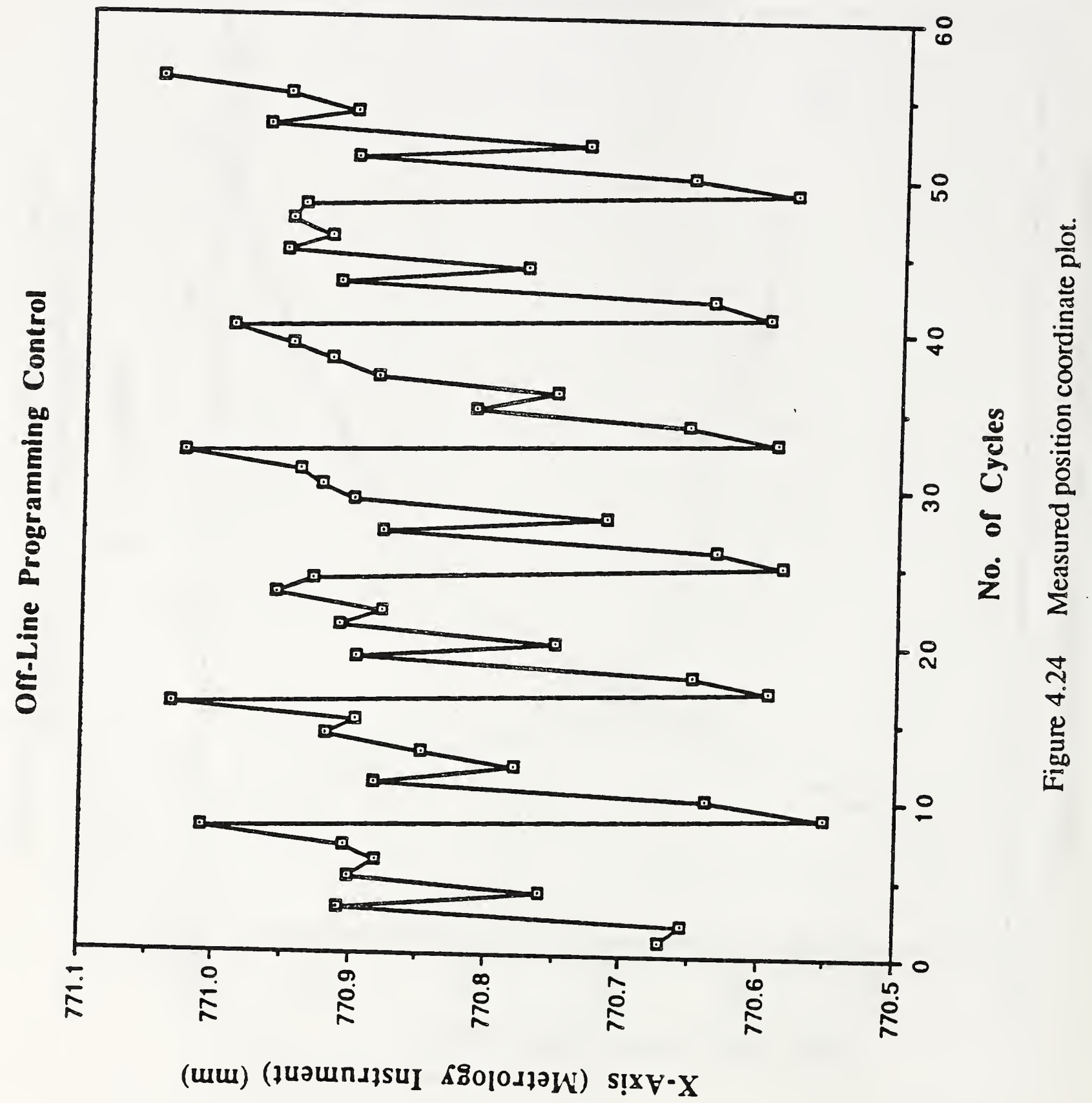




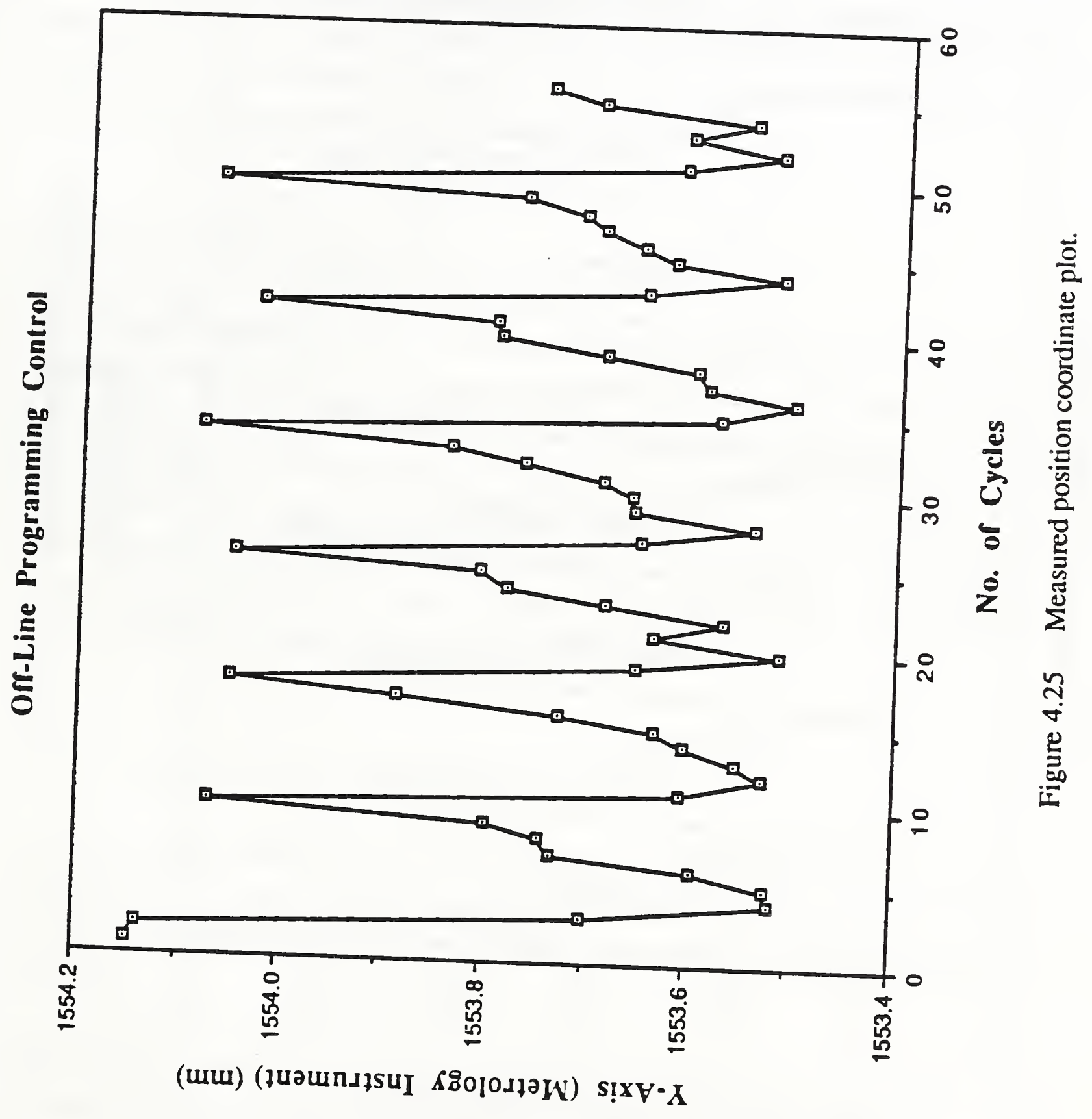




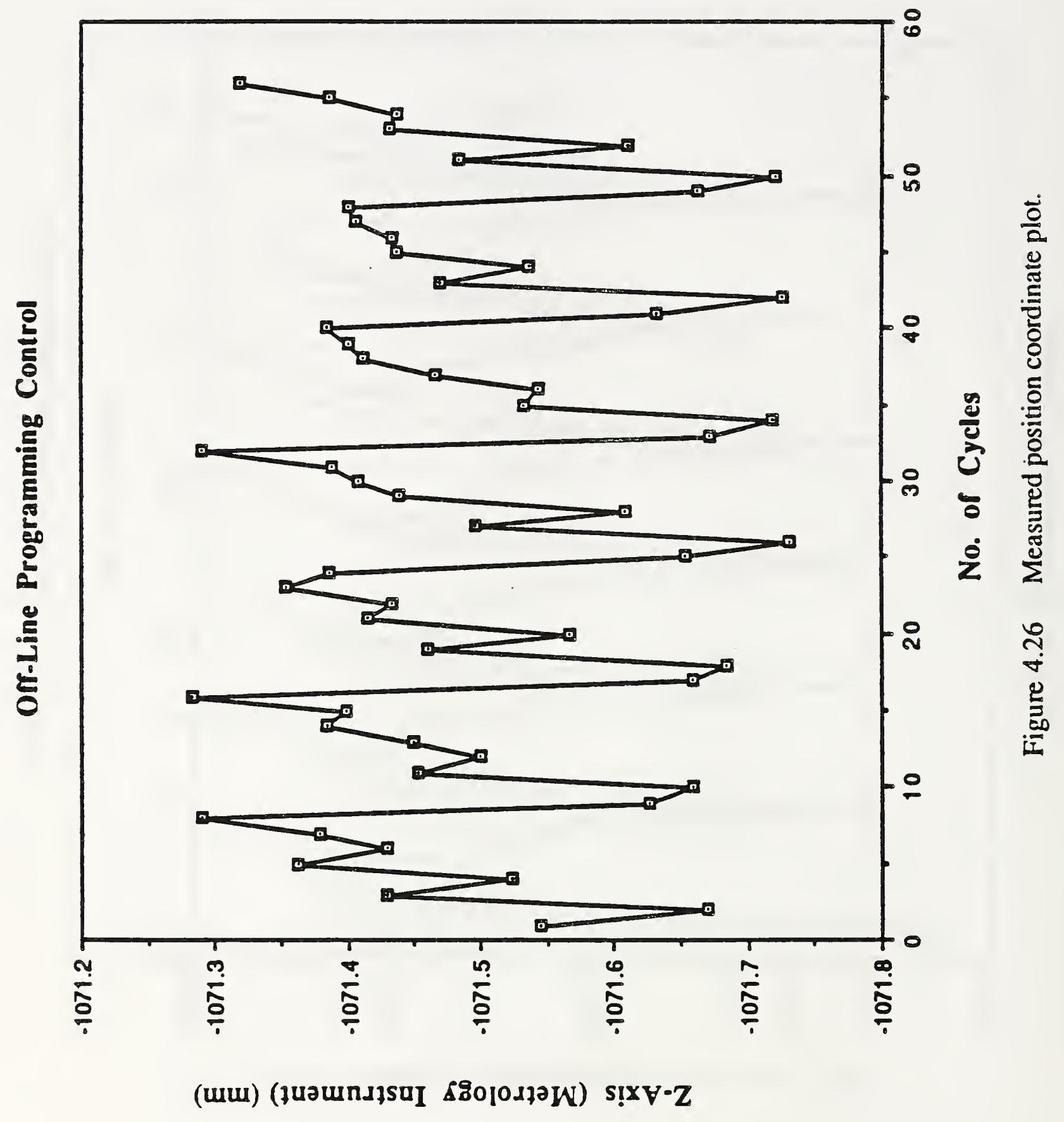


superimposed. Is that the result of the robot position control algorithm used, which does not stop servoing for as long as there is a joint angle position error? This of course raises the question, what would happen if only one initial position was used, would the random component dominate the achieved position? The test which will be reported in the next section will try to answer that question.

Comparing the errors, which are measured when the teach mode joint angles kinematics control is used and when off-line programming control is used, it can be seen that in the second case they are larger for both accuracy and repeatability, and the peak-to-peak amplitudes of the coordinates oscillations. This makes sense since in that case the errors due to the inverse kinematics algorithms are also included.

\subsubsection{2.a Limited motion off-line programming}

The off-line programming tests were repeated, eliminating the motions to and from the different initial positions. By comparing the results of this test with those of the previous test the effect of moving from different initial positions can be determined. For each repetition of this test, the robot moved between $S 2_{\text {app }}$ and $S 2$ eight times, using Cartesianinterpolated motion. Again, the position data was recorded each time the robot reached S2 and the test was repeated seven times, resulting in 56 data points. The motion time between $S 2_{\text {app }}$ and $S 2$ was the same as that used for the previous tests. Each repetition of the sequence of eight points took just over 3 min for this test.

\subsubsection{2.b Analysis and Conclusions}

The laser tracker coordinates of the 56 measured positions were again divided into 7 analysis groups. The data contained in each group were analyzed separately and the results of the analysis were used to determine the effect of the number of measured positions on the results.

Table 4.6 in the Appendix shows the results of the analysis of the last group of data which contains all 56 measured positions. Figure 4.27 is a three dimensional plot of the measured achieved positions (triangular marks), their mean position (cross mark), and the 


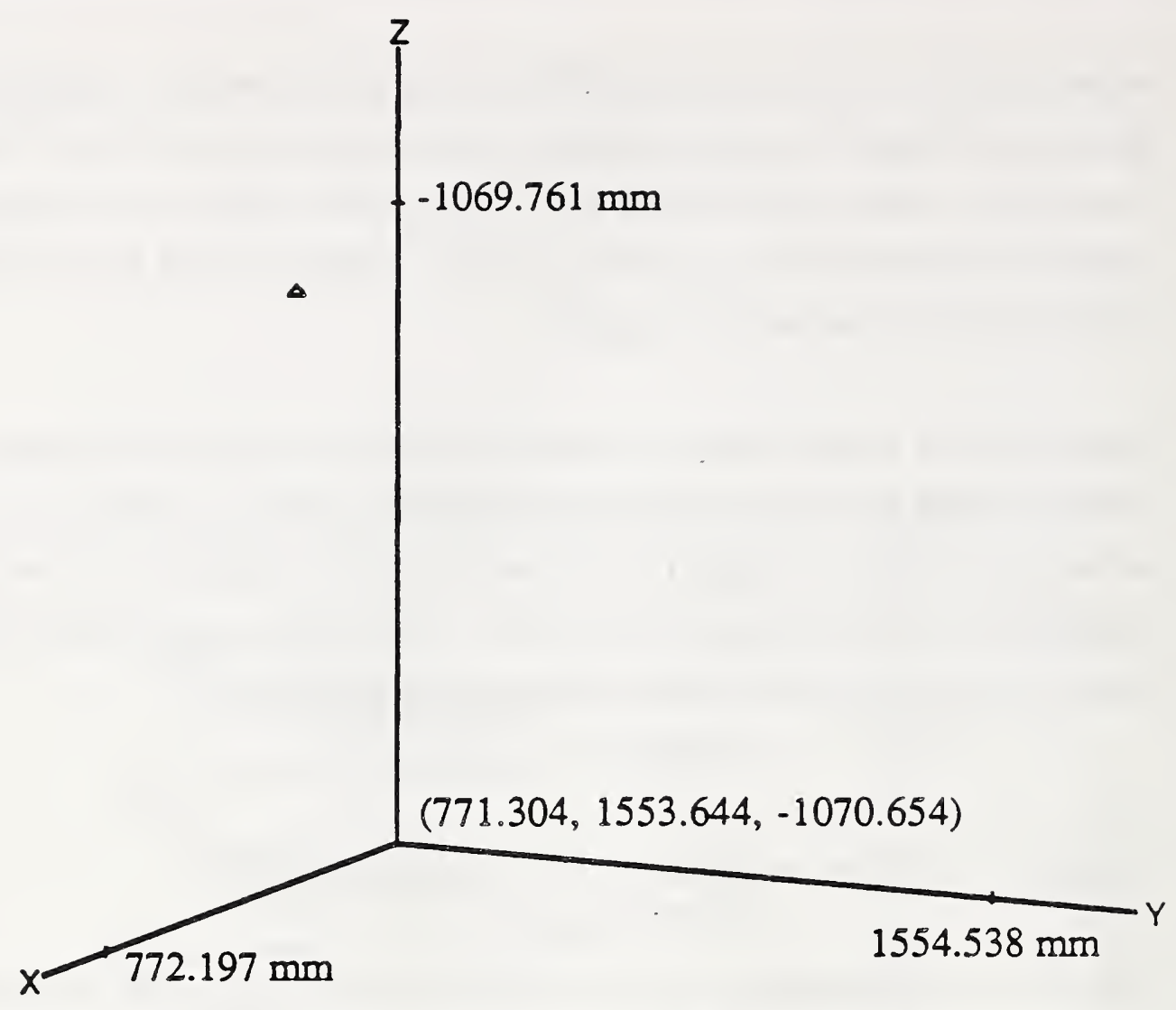

$\Delta$

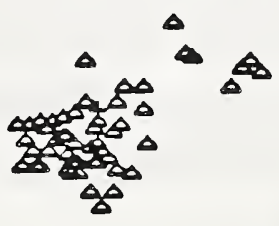

ם

Plot of the PTP Test Achieved Positions

Each triangle is located at an achieved position

The square marks the commanded position

The cross marks the mean of the achieved positions

Figure 4.27 Limited motion off-line programming positions plot. 
commanded position (square mark). The coordinate frame in that figure is that of the laser tracker after it was translated to the centroid of those positions.

Figure 4.28 is a plot of the same positions as they are projected on a plane defined by the $\mathrm{X}$ and $\mathrm{Z}$ coordinate axes. As can be seen the cluster of points forms again a galactic cloud with an orientation which is approximately orthogonal to the orientation of the axis of the first joint of the robot arm. In this case, though, the first two points in that plot can be seen to be located away from the rest, which cluster together in a tight group. The coordinates of those points correspond to the first two cycles of the test and the corresponding achieved positions. From Table 4.6 it can be seen that the first achieved position is approximately $1.0 \mathrm{~mm}$ away from the rest along both the $\mathrm{Z}$ and $\mathrm{X}$ axes. It is higher than the rest along the $\mathrm{Z}$-axis direction, and forward along the $\mathrm{X}$-axis direction.

Figure 4.29 is a plot of the ISO defined accuracy error versus the number of cycles contained in each analysis group. Figure 4.30 is a plot of the ISO defined repeatability error versus the number of cycles contained in each analysis group. As can be seen from these plots the errors seem to decrease exponentially reaching an asymptote after 40 to 56 cycles. The values of these errors start from rather high values as compared to those from the previous section (Figures 4.22, 4.23), probably because of the difference in the coordinates of the achieved positions of the first two cycles as compared to the rest. The level of the asymptotes is higher too.

To better understand the nature of this decrease of the accuracy and repeatability errors the $\mathrm{X}, \mathrm{Y}$, and $\mathrm{Z}$ axes, laser tracker coordinates of the 56 measured positions were plotted as a function of the number of cycles and are shown in Figures 4.31, 4.32, 4.33 respectively. These plots show again a periodicity with a fundamental frequency of 8 cycles and a few smaller amplitude higher frequency oscillations. There is a significant drift during the first 8 cycles, which corresponds to the first group of analysis data. After the first 8 cycles the coordinates seem to follow a relatively stable periodic oscillation. This significant drift at the beginning of the test is due mainly to the achieved positions during the first two cycles which are far away (especially the first one) from the rest. The periodic oscillations of the achieved positions are unexpected in this case, since all moves initiate from the same position (the approach position).

The off-line programming limited motion test was repeated four more times under various operating conditions to determine the source of the periodic oscillations and whether the 

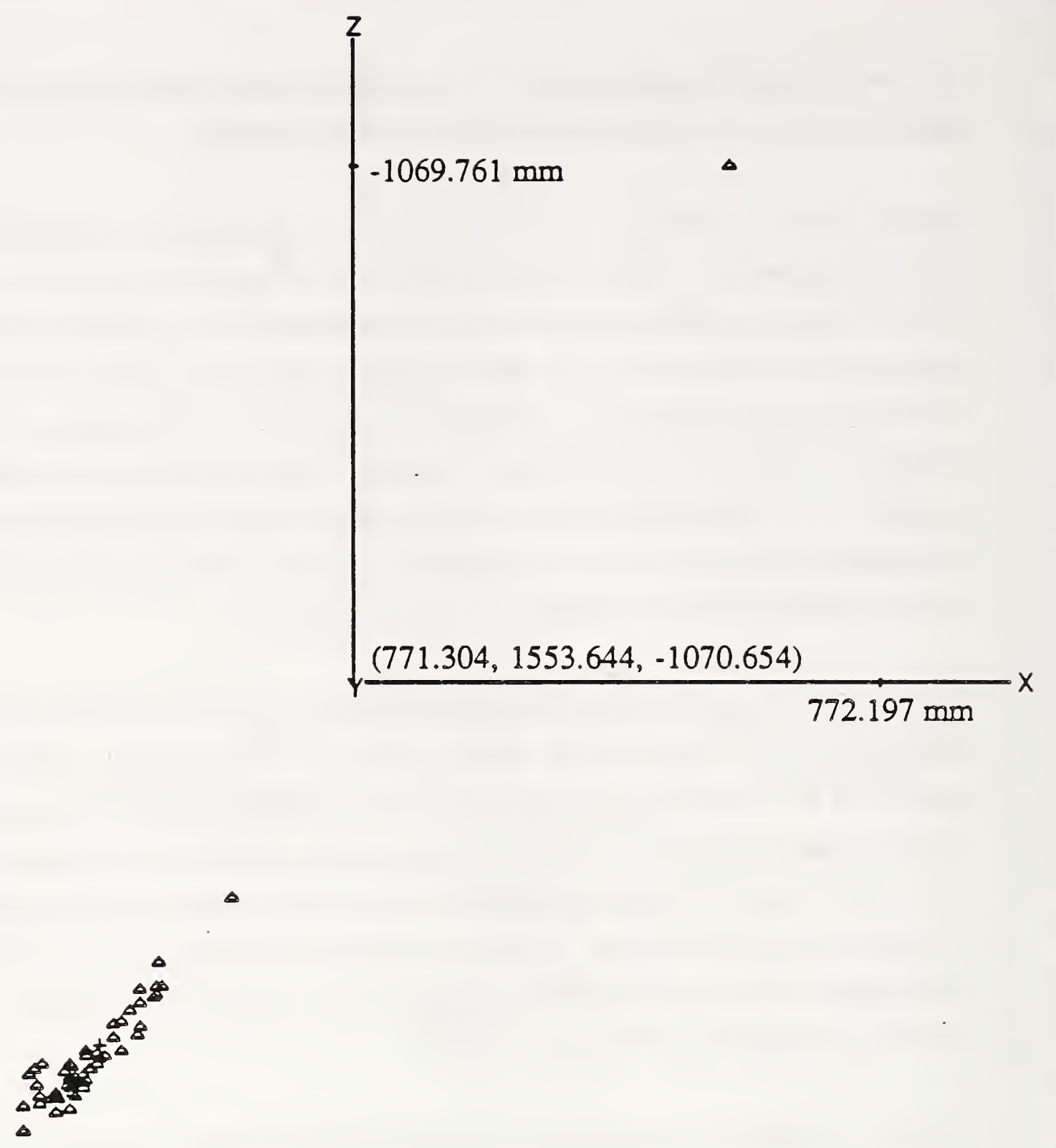

0

Plot of the PTP Test Achieved Positions

Each triangle is located at an achieved position

The square marks the commanded position

The cross marks the mean of the achieved positions

Figure 4.28 Limited motion off-line programming positions plot. 


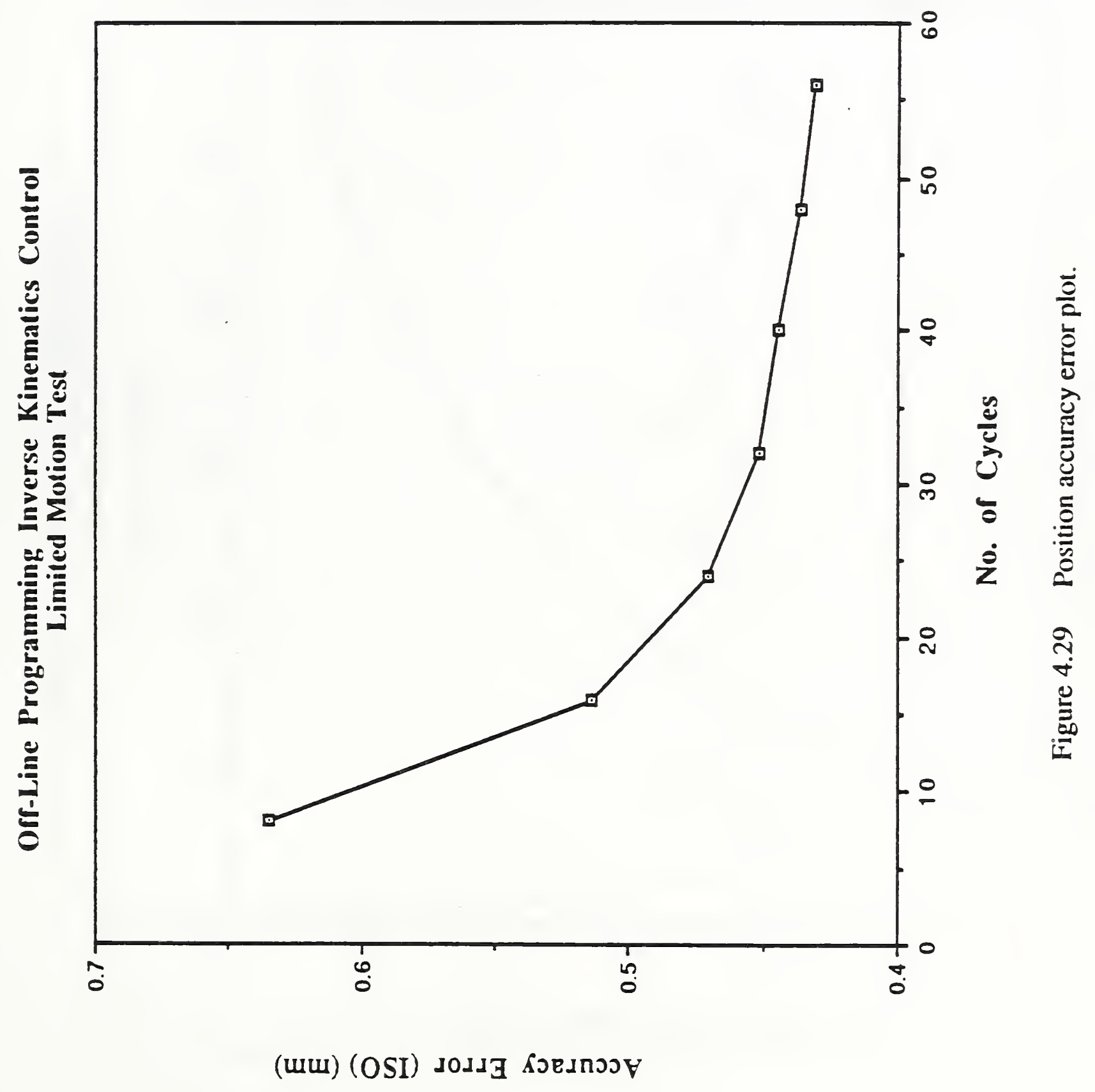




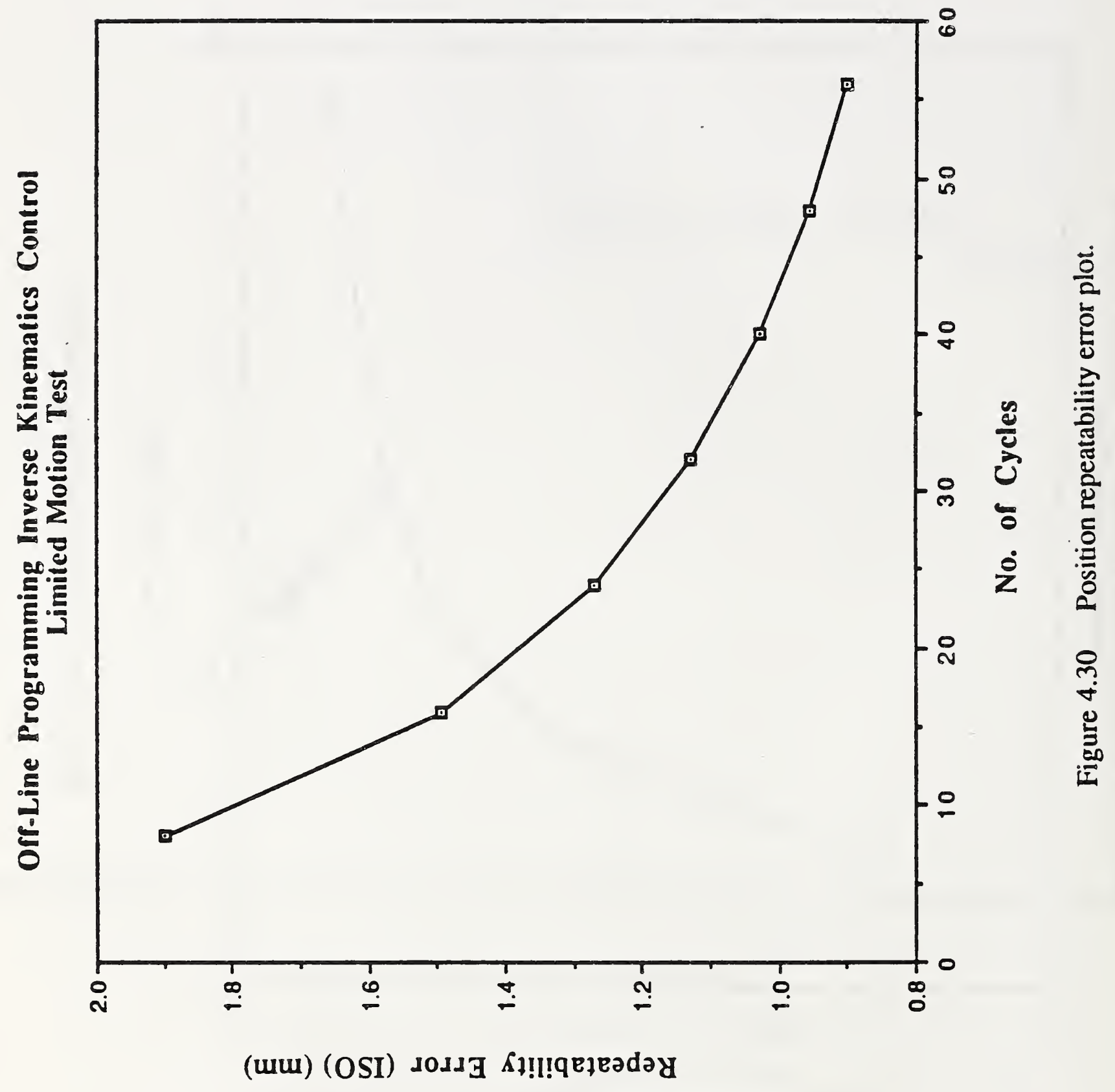




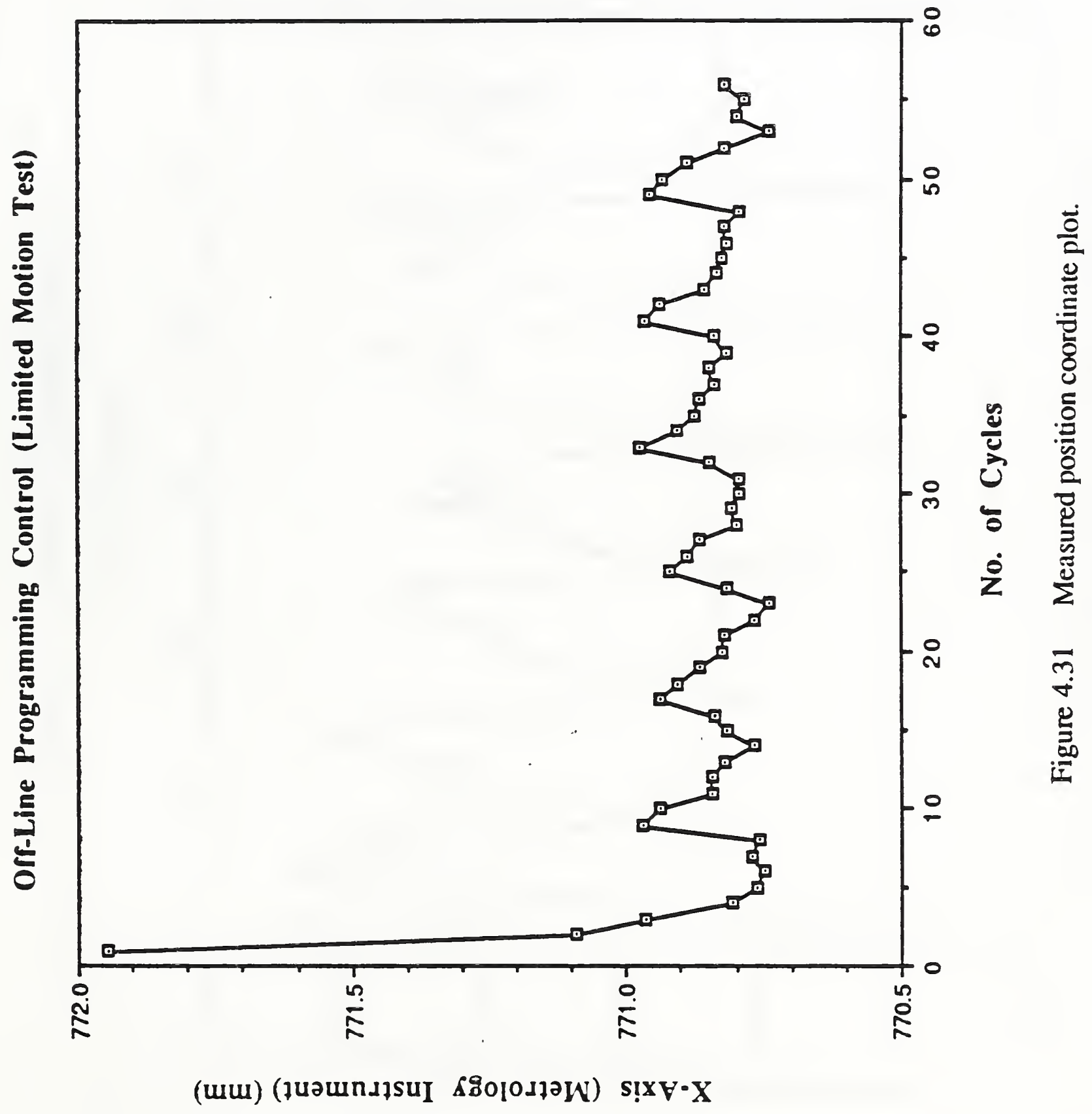




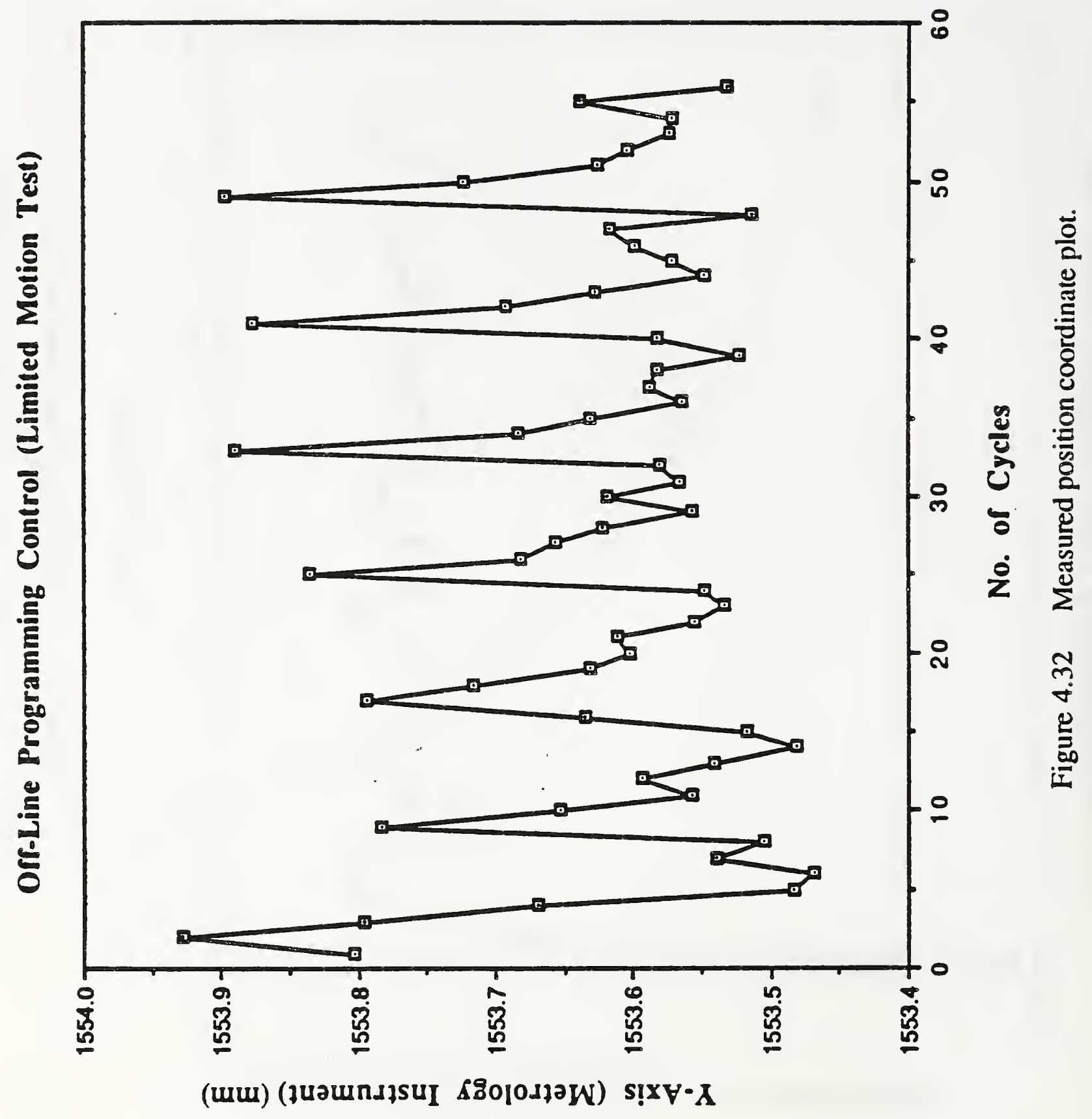




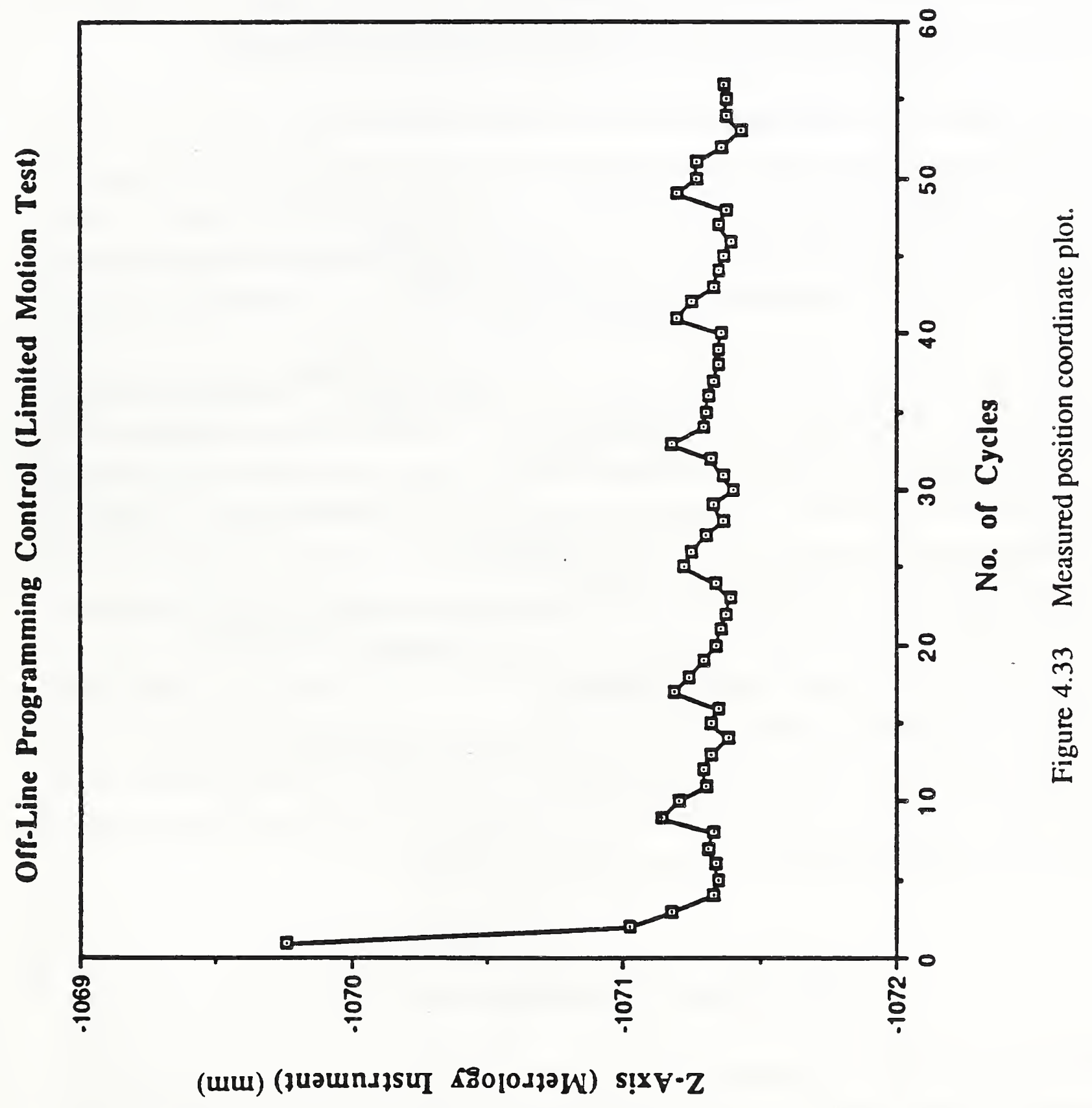


large position error during the first few cycles is accidental or not. In each test 120 cycles of motion were recorded. In the first case (test \#2), the test conditions were the same as those used in the previous tests. In the second case (test \#3), a time delay between every 8 cycles, of approximately 10 seconds was eliminated, because it was thought that it might be responsible for the periodic oscillations. In the third case (test \#4), in addition to the time delay between every 8 cycles being eliminated, the integral gain of the joint servo control was reduced to zero. In the fourth case (test \#5), an additional delay of less than 100 mseconds, for resetting the command file every 8 cycles was also eliminated.

As can be seen from these plots, as soon as the integral controller gain was set to zero both the periodic oscillations and the large position error during the first few cycles disappears, while a position drift has now been added. Figures 4.34, 4.35, 4.36, show the laser tracker $\mathrm{X}, \mathrm{Y}, \mathrm{Z}$, coordinates for the \#4 test (no large time delay, no integral control). Ignoring the drift, the measured achieved positions seem to be distributed rather randomly.

Comparing the results of the off-line programming test for motions from the standard RIA path positions (see Figures 4.22, 4.23) with those for limited motions (see Figures 4.29, 4.30), it can be seen that the errors measured for the limited motion case are larger for both accuracy and repeatability. The peak-to-peak amplitudes of the coordinates oscillations probably cannot be compared because of the large position error during the first few cycles of the limited motion off-line programming control case. From the test data discussed so far it appears that the limited motion off-line programming test cannot be used as a substitute of the standard RIA path positions off-line programming test, because the behavior of the robot arm seems to be quite different for these two cases. The effect of the integral gain, time delays and travel distance on performance has to be studied more carefully. Preliminary results from tests with shorter travel distances and no integral control show a significant drift over the duration of the tests.

\subsubsection{Robot Position Resolution Tests}

The objectives of the resolution tests were to observe and measure the ability of a robot arm to move its end-effector by small increments in specific directions and the effect of the number of measured increments on the results. Since, to the best of our knowledge, no established robot position resolution tests exist, a simple test and metric were established for the work reported here. Three orthogonal directions of motion, parallel to the robot arm 


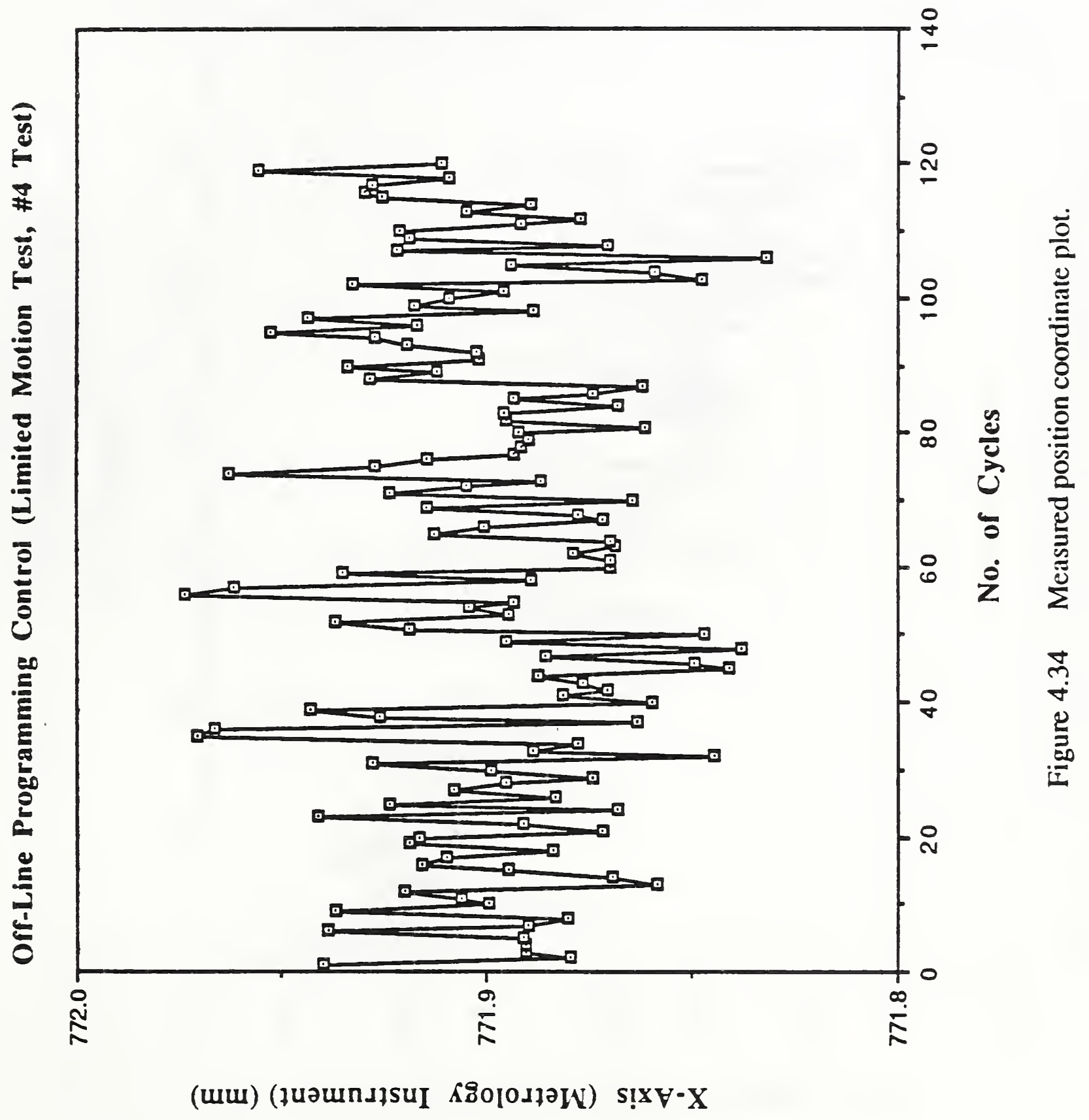




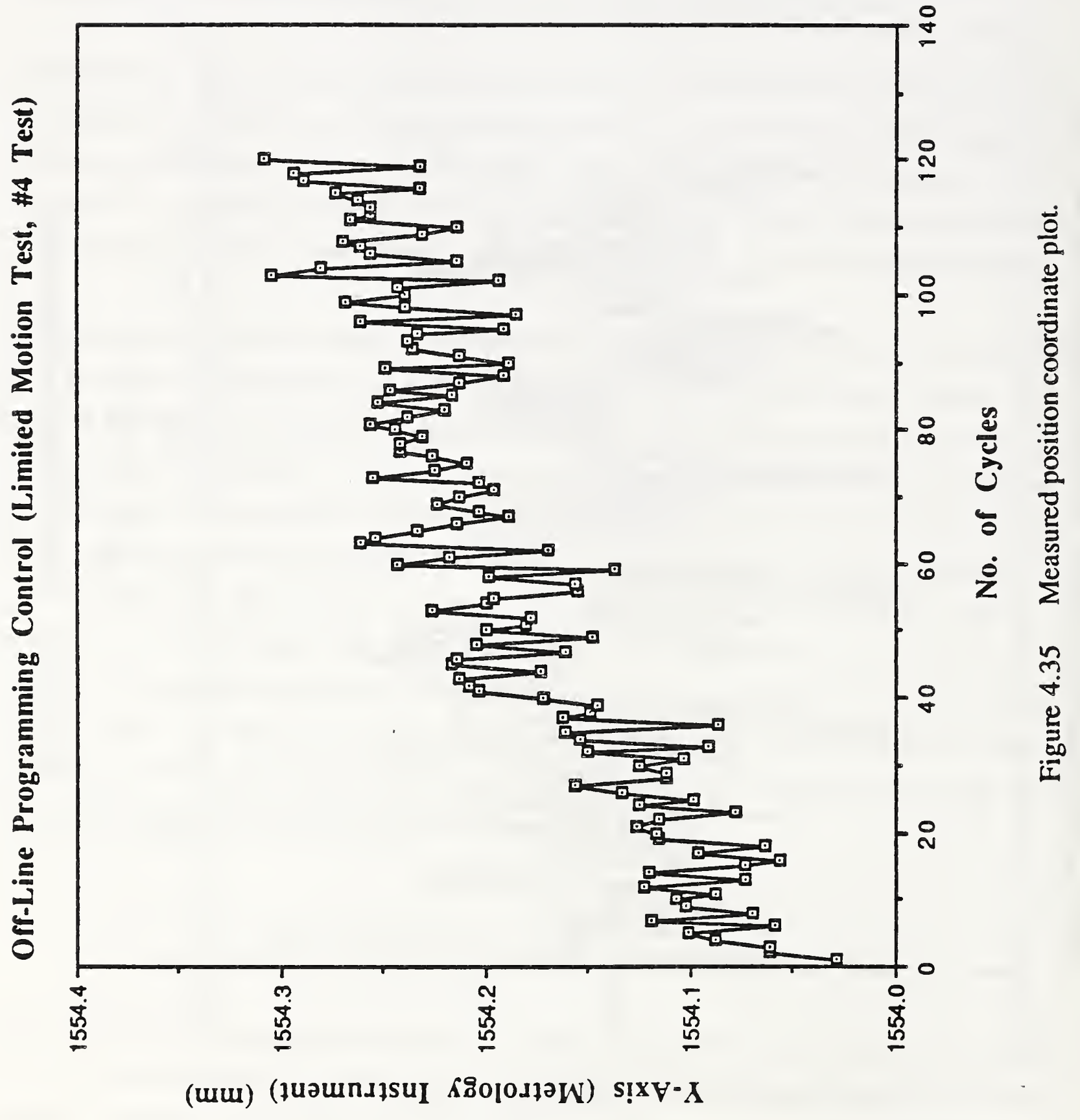




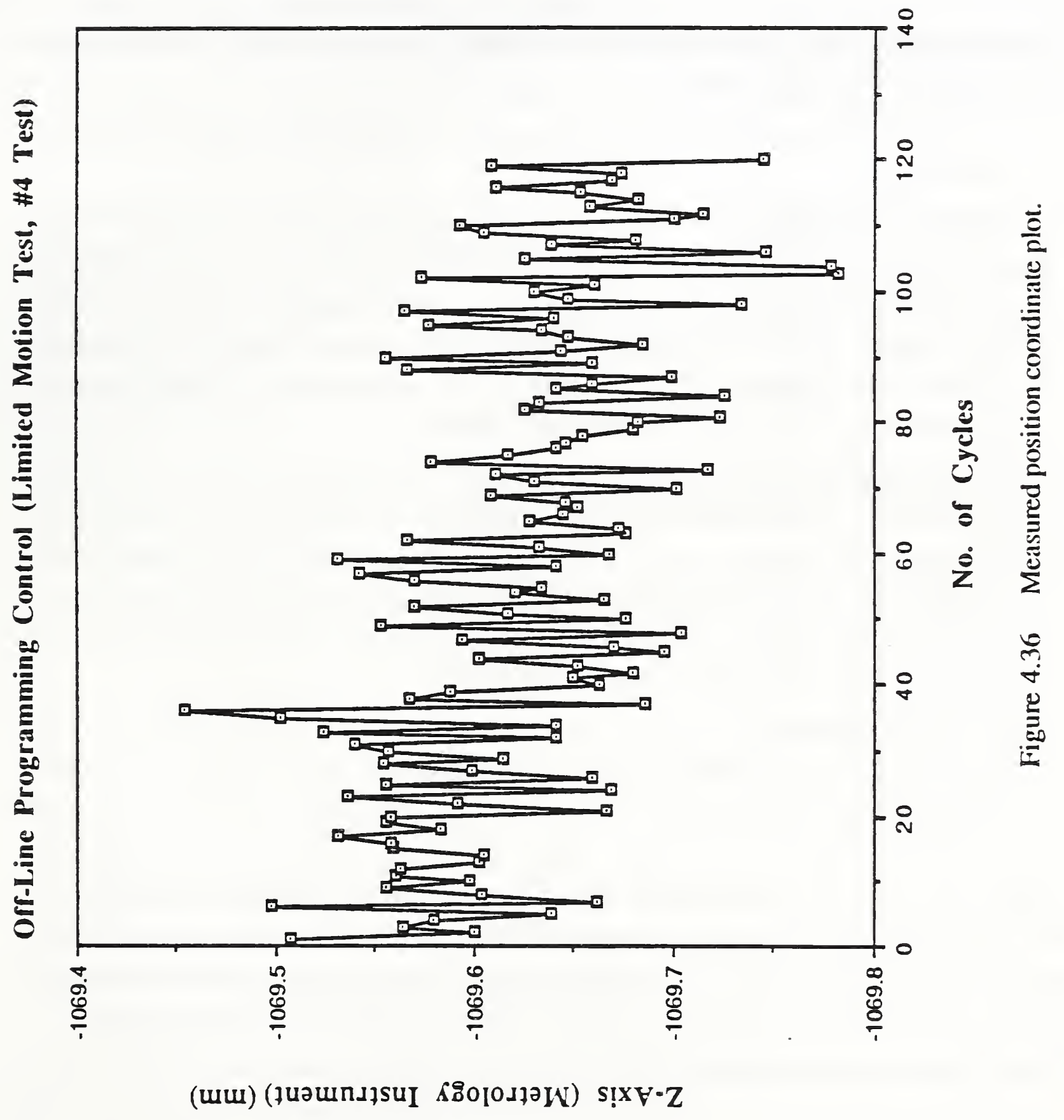


baseframe coordinate axes, with the simulated sensor nest location as the nominal position, were selected for the resolution test incremental moves. Increments of various lengths were tested. Due to the small size of the allowable workspace a forward and backward incremental motion had to be used.

\subsection{4.a Tests}

The purpose of this test is to determine how well the robot can move very small distances. The procedure for this test is as follows:

Move to S2

Record position data

Move to $\mathrm{S} 2+0.150 \mathrm{~mm}$ in the world $\mathrm{X}$ direction

Record position data

Move to $\mathrm{S} 2+0.300 \mathrm{~mm}$ in the world $\mathrm{X}$ direction

Record position data

Move to $\mathrm{S} 2+0.450 \mathrm{~mm}$ in the world $\mathrm{X}$ direction

Record position data

Move to $\mathrm{S} 2+0.600 \mathrm{~mm}$ in the world $\mathrm{X}$ direction

Record position data

Move to $\mathrm{S} 2+0.450 \mathrm{~mm}$ in the world $\mathrm{X}$ direction

Record position data

Move to $\mathrm{S} 2+0.300 \mathrm{~mm}$ in the world $\mathrm{X}$ direction

Record position data

Move to S2 $+0.150 \mathrm{~mm}$ in the world X direction

Record position data

A motion time of $5 \mathrm{~s}$ was specified for each of these incremental motions. This sequence was repeated seven times, and then the same test was performed for incremental motions in the world $\mathrm{Y}$ and $\mathrm{Z}$ directions. Each sequence for each direction requires just over $1 \mathrm{~min}$ to perform. Total time for seven repetitions for all directions (168 points) is approximately 23 min. Cartesian trajectories were used for all motions. 


\subsection{4.b Analysis and Conclusions}

The laser tracker coordinates of the 56 measured positions in each orthogonal direction of movement were again divided into 7 analysis groups. The data contained in each group were analyzed separately and the results of the analysis were used to determine the effect of the number of measured positions on the results.

The mean and the standard deviation of the magnitudes of the increments in each direction and for each analysis group were calculated. Table 4.7 in the Appendix shows the results of the analysis of the last group of data which contains all 56 measured positions in each orthogonal direction of movement, for commanded incremental moves of $0.15 \mathrm{~mm}$.

Figure 4.37 is a three dimensional plot of the measured achieved positions (cross marks for the $\mathrm{X}$-direction moves, square marks for the $\mathrm{Y}$-direction moves and triangle marks for the Z-direction moves). The coordinate frame in that figure is that of the laser tracker after it was translated to the centroid of those positions. As can be seen from that figure the incremental moves in each direction are not of equal length or direction.

Figures $4.38,4.39,4.40$ are plots of the mean values of the measured magnitudes of the increments in the three directions of motion, versus the number of cycles contained in each analysis group. As can be seen from these plots, the mean values seem to decrease and approach an asymptote after 24 to 32 cycles for the data coming from the $\mathrm{X}$ and $\mathrm{Z}$-axes directions of movement, but not for the $\mathrm{Y}$-axis direction of movement.

The $0.15 \mathrm{~mm}$ incremental motion tests gave very questionable results, raising doubts whether this robot arm and controller can move in increments that small in specific directions. To check whether the situation improves with larger size increments the test was repeated with increments of $0.5 \mathrm{~mm}$ length. Table 4.8 in the Appendix shows the results of the analysis of the last group of data which contains all 56 measured positions in each orthogonal direction of movement, for commanded incremental moves of $0.5 \mathrm{~mm}$. Figure 4.41 is a three dimensional plot of the measured achieved positions.

Comparing the plots from Figures 4.37 and 4.41 it can be seen that no significant improvement in the regularity of the magnitude and straightness of the incremental moves has been achieved. This of course raises the question of whether the mean value of the magnitudes of the increments is a sufficient measure for characterizing robot arm 


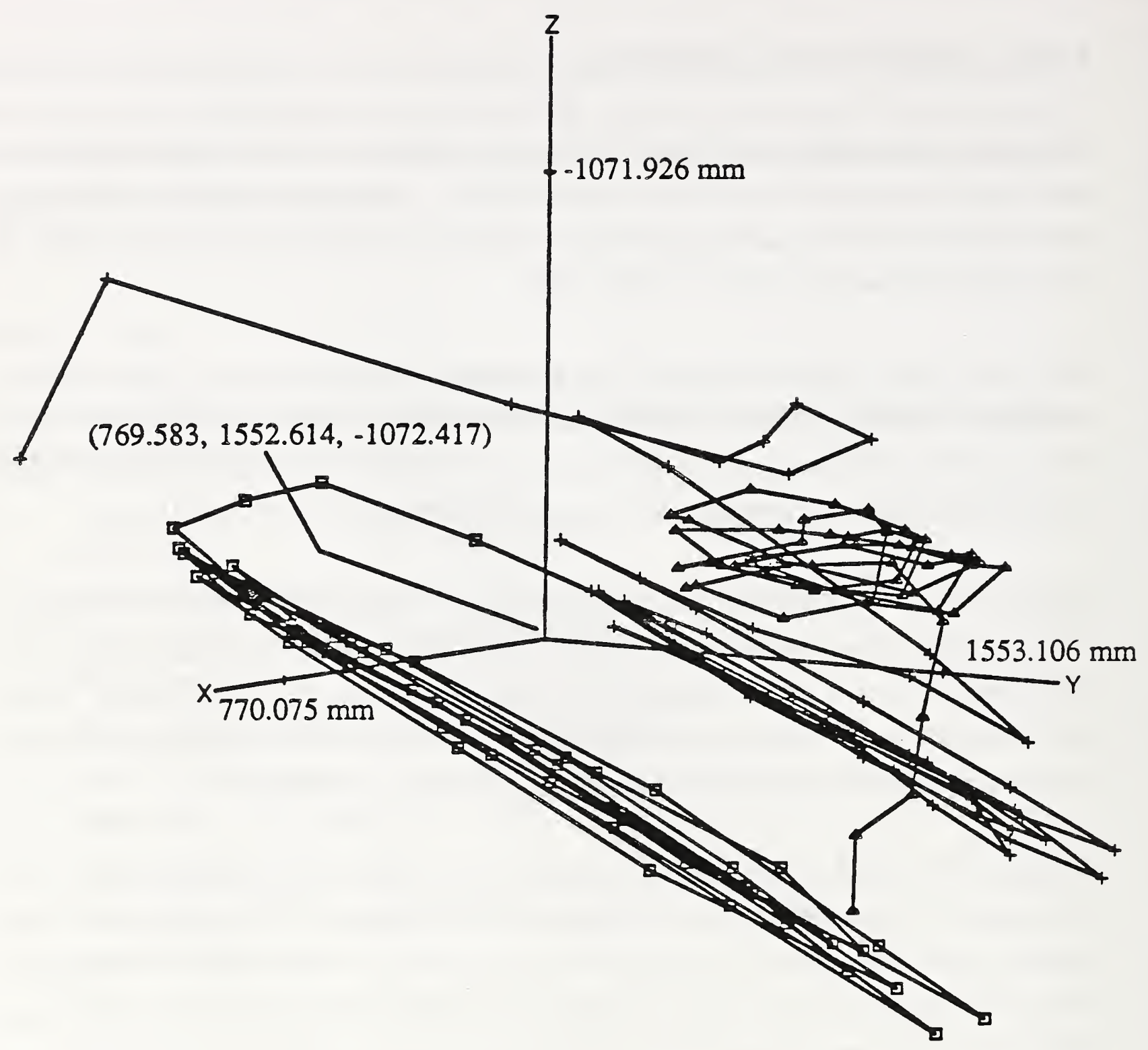

The cross marks the $\mathrm{X}$-direction move

The square marks the $Y$-direction move

The triangle marks the $Z$-direction move

Figure 4.37 Robot resolution test positions. 


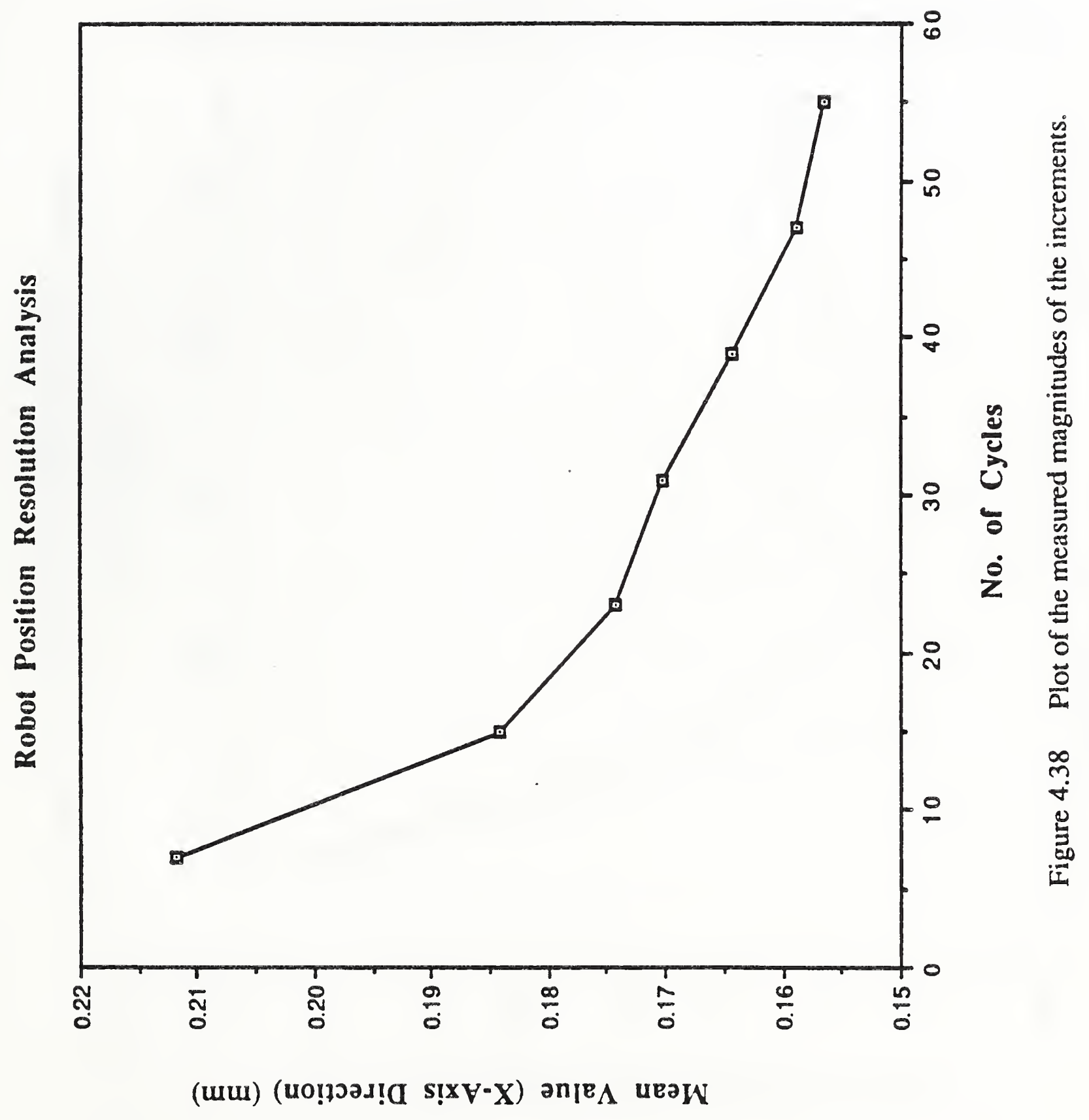




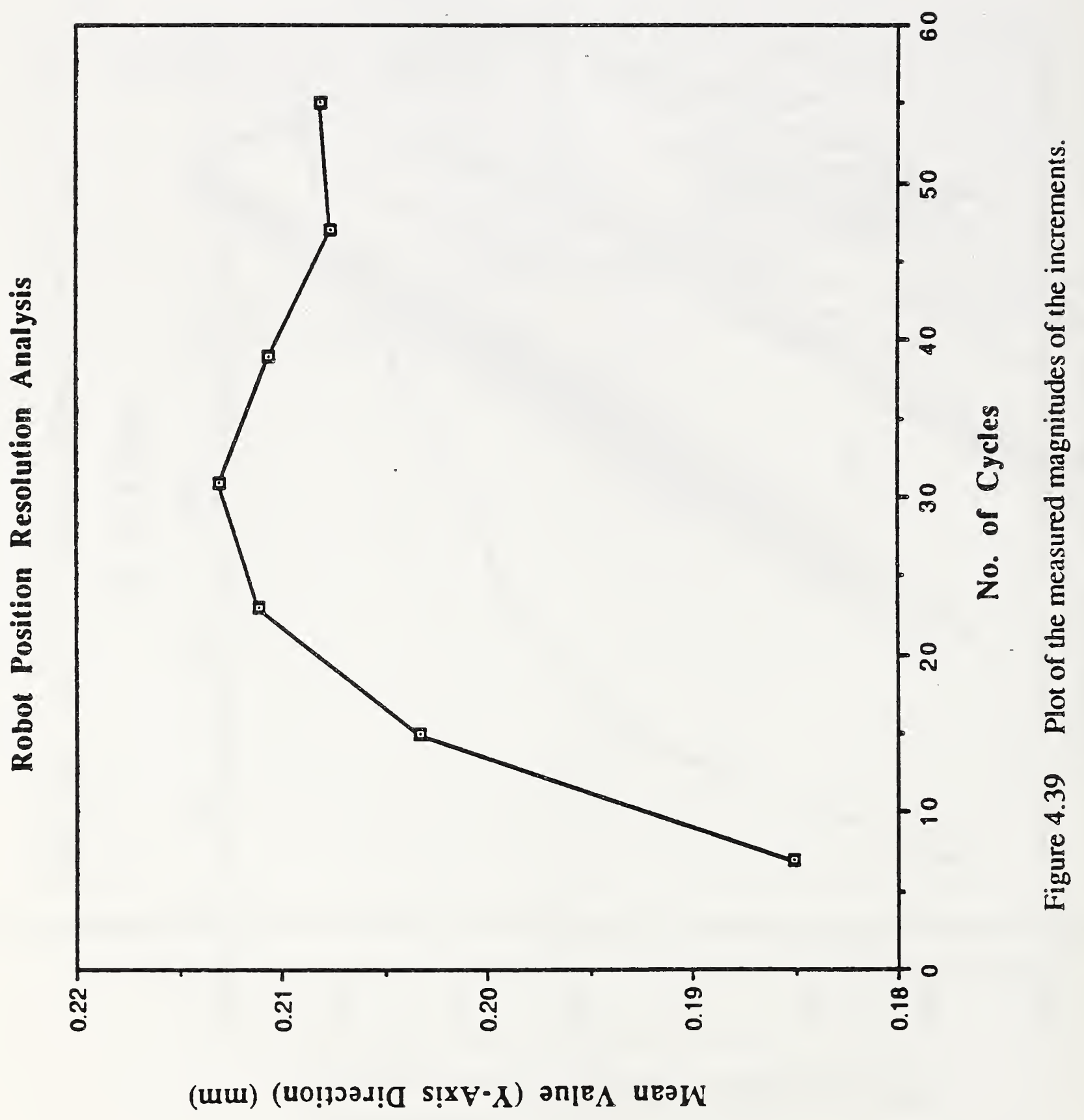




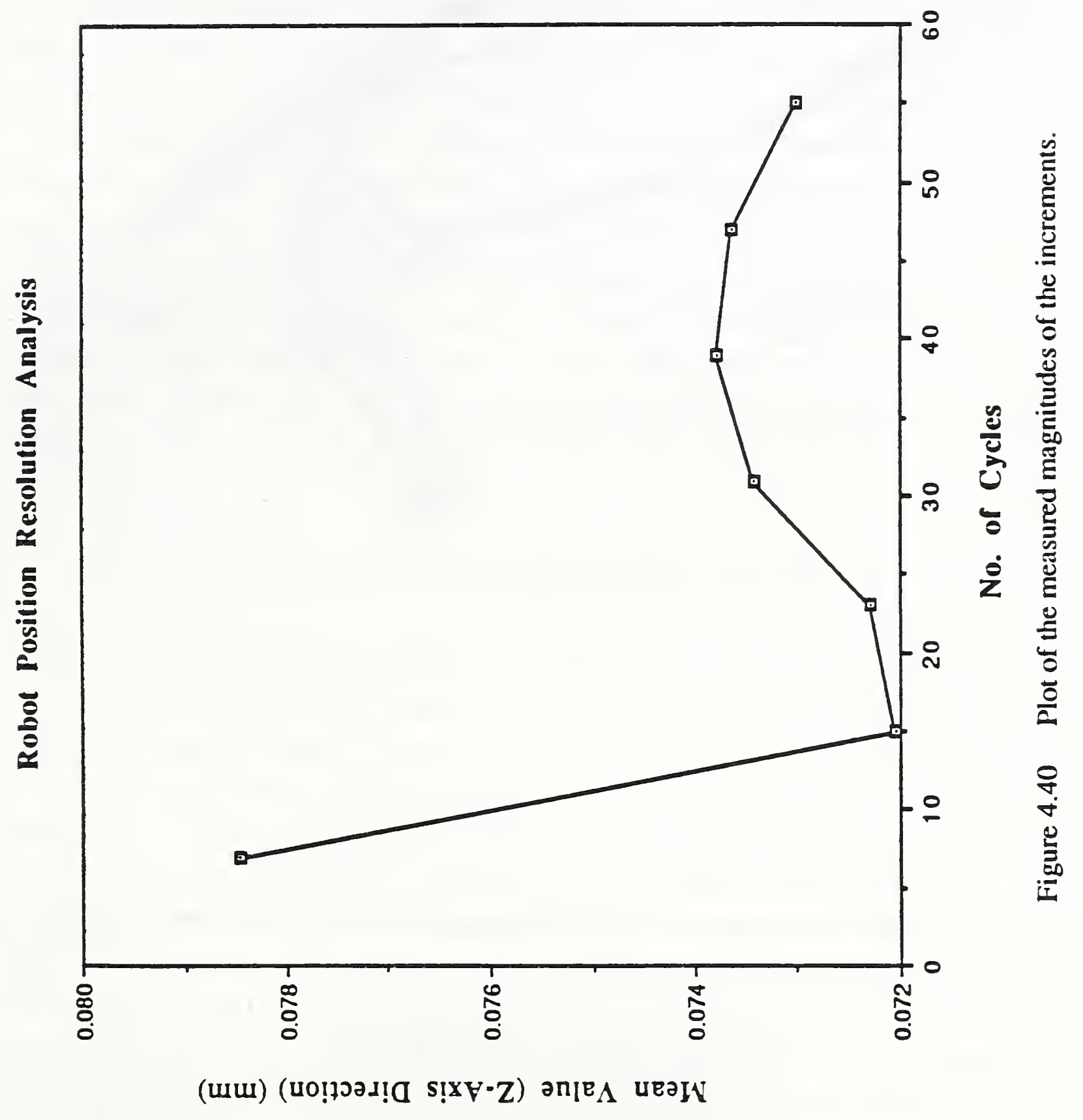




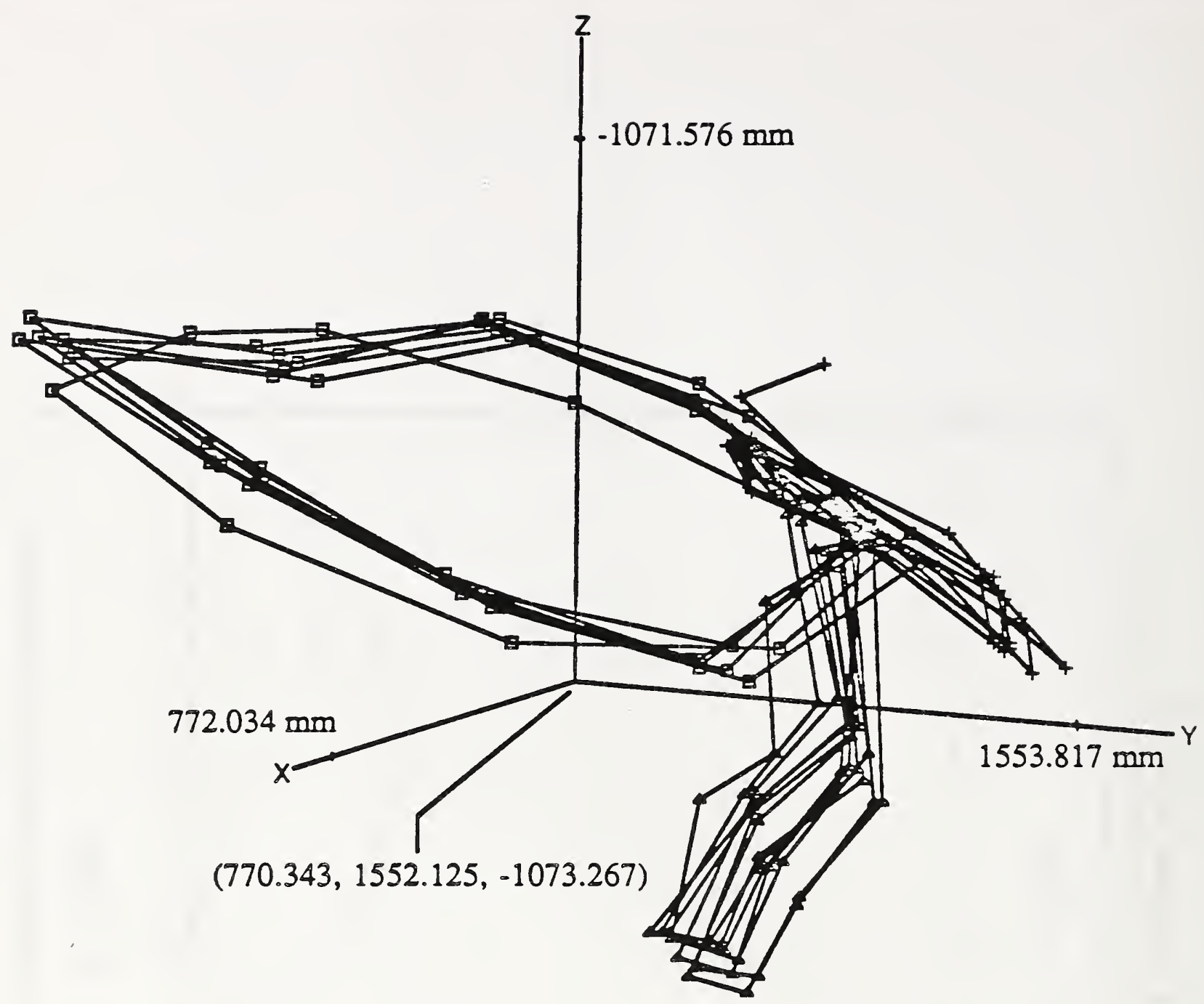

The cross marks the $\mathrm{X}$-direction move

The square marks the $Y$-direction move

The triangle marks the $Z$-direction move

Figure 4.41 Robot resolution test positions. 
resolution. Perhaps if the mean value of the orientation error, with respect to the commanded direction of move is included, a more complete description of the measured achieved move will be provided. Another more compact way of characterizing robot arm resolution would be to provide the mean value of the projection of the measured achieved motion along the direction of commanded move.

In general, very small motions along orthogonal baseframe coordinate axes will be difficult for a serial revolute arm to perform. This is because in most cases the motion of the arm joints contribute in a complex way to Cartesian motion of the end effector, and joint-related disturbances, such as stiction, become significant. Due to the smallness of the motion the torque commands are initially not sufficient to overcome stiction. If an integral control term is used, it will sense the error and build up the torque until motion initiates, then the arm will probably overshoot the commanded position and the whole process will be repeated again. Some possibilities for improving the incremental positioning resolution include modifying the servo gains, and adding dither or other friction compensation torques to the control. A more detailed study of this small torque-small displacement interaction would have to be performed on the robot arm being tested to better understand the resolution error problem.

\subsection{Forward Kinematics Error Analysis}

Under joint interpolated teach mode control the robot control system servo algorithm, combined with the torque loop control, motor amplifiers, joint drives, and joint position and torque sensors, causes the robot to move the joints to prerecorded angles. Therefore the teach mode control performance tests may reveal defects of any of the above components. Under off-line programming the robot control system must also use the inverse kinematics algorithms to determine the joint angles which correspond to the commanded Cartesian coordinates. These angles then become the commands which are sent to the servo level. Therefore the off-line programming performance tests may reveal defects of the inverse kinematics algorithms as well as the servo algorithm, the mechanical part of the robot arm, and other servo components. The objective of the analysis described in this section was to test the performance of the forward kinematics model and algorithms. Forward kinematics algorithms are used by many new sophisticated robot controllers for compliance control, precision move control, calibration, etc. 
No new tests had to be performed for the forward kinematics analysis work; any test data which included target position information measured by the laser tracker and the robot controller could be used for the purposes of this analysis. The idea behind this analysis is to use the coordinates transformation to convert the laser tracker measured coordinates to robot controller coordinates, then compare these converted coordinates to the robot controller calculated coordinates for the same target positions. The differences in the two sets of coordinates are due to errors in the forward kinematics algorithms of the robot controller and the laser tracker controller, since they are the ones used by the controllers to convert from joint positions to target Cartesian coordinates. Equations 4.2 to 4.13 are then used to calculate the accuracy and repeatability errors, where in this case the coordinates of the achieved positions are the coordinates of the calculated errors and the coordinates of the commanded position are all zeroes, which are the desired values of the errors.

One of the major sources of robot kinematic modelling errors is the joint position initialization error. When the robot power is turned on its controller has to find the precise location of its joints. Every time it does that a small bias error is added to the joint position estimate. The combination of these errors results in a small translation and rotation of the robot baseframe every time the power is turned off and on. The forward kinematics error analysis can be used to estimate the contribution of this initialization error to the position accuracy and repeatability errors. If the position data used to estimate the coordinates transformation and the forward kinematics errors are collected before the robot power is turned off, there will be no contribution from the joint position initialization offset error. This is true because the coordinates transformation includes the translation and rotation of the robot baseframe due to that error. If, on the other hand, the target positions are collected after the power is turned off and on, any difference in the joint position initialization will contribute to the measured performance errors.

To determine an estimate of the initialization error, two sets of data were analyzed. The first was collected at about the same time the coordinates transformation data were collected (with no reinitialization), while the second was collected the next day after the robot arm power was turned off and on a couple of times. Of course, every effort was made to keep all the other conditions of the experiment approximately the same, like the room temperature, the amount of robot arm exercise, etc. The analysis of these two sets of data are presented in the following sections. 


\subsubsection{Continuous operation error analysis and Conclusions}

The test data used for this analysis are those of the teach mode inverse kinematics test listed in Table 4.2. At the end of that test and before the robot arm power was turned off data from 14 positions were collected in order to determine the mathematical transformation between the coordinate frame of the laser tracker and the robot baseframe. It is this transformation listed in Table 4.3 that was used to convert the data used for this analysis.

The laser tracker coordinates of the 56 measured positions were again divided into 7 analysis groups. The data contained in each group were analyzed separately and the results of the analysis were used to determine the effect of the number of measured positions on the results.

Table 4.9 in the Appendix shows the results of the analysis of the last group of data which contains all 56 measured positions. Figure 4.42 is a three dimensional plot of the calculated forward kinematics errors (triangular marks), and their mean (cross mark).

Figure 4.43 is a plot of the ISO defined accuracy error versus the number of cycles contained in each analysis group. Figure 4.44 is a plot of the ISO defined repeatability error versus the number of cycles contained in each analysis group. As can be seen from these plots the values of both of these errors are small compared to the errors measured from all the previous tests. The variation in their values as a function of the number of cycles is very small too.

\subsubsection{Interrupted operation error analysis and Conclusions}

The test data used for this analysis are those of the teach mode joint angles kinematics test listed in Table 4.1. The same transformation used to convert the data of the continuous operation forward kinematics error analysis was used for these data too. This is the transformation listed in Table 4.3.

The laser tracker coordinates of the 56 measured positions were again divided into 7 analysis groups. The data contained in each group were analyzed separately and the results of the analysis were used to determine the effect of the number of measured positions on the results. 


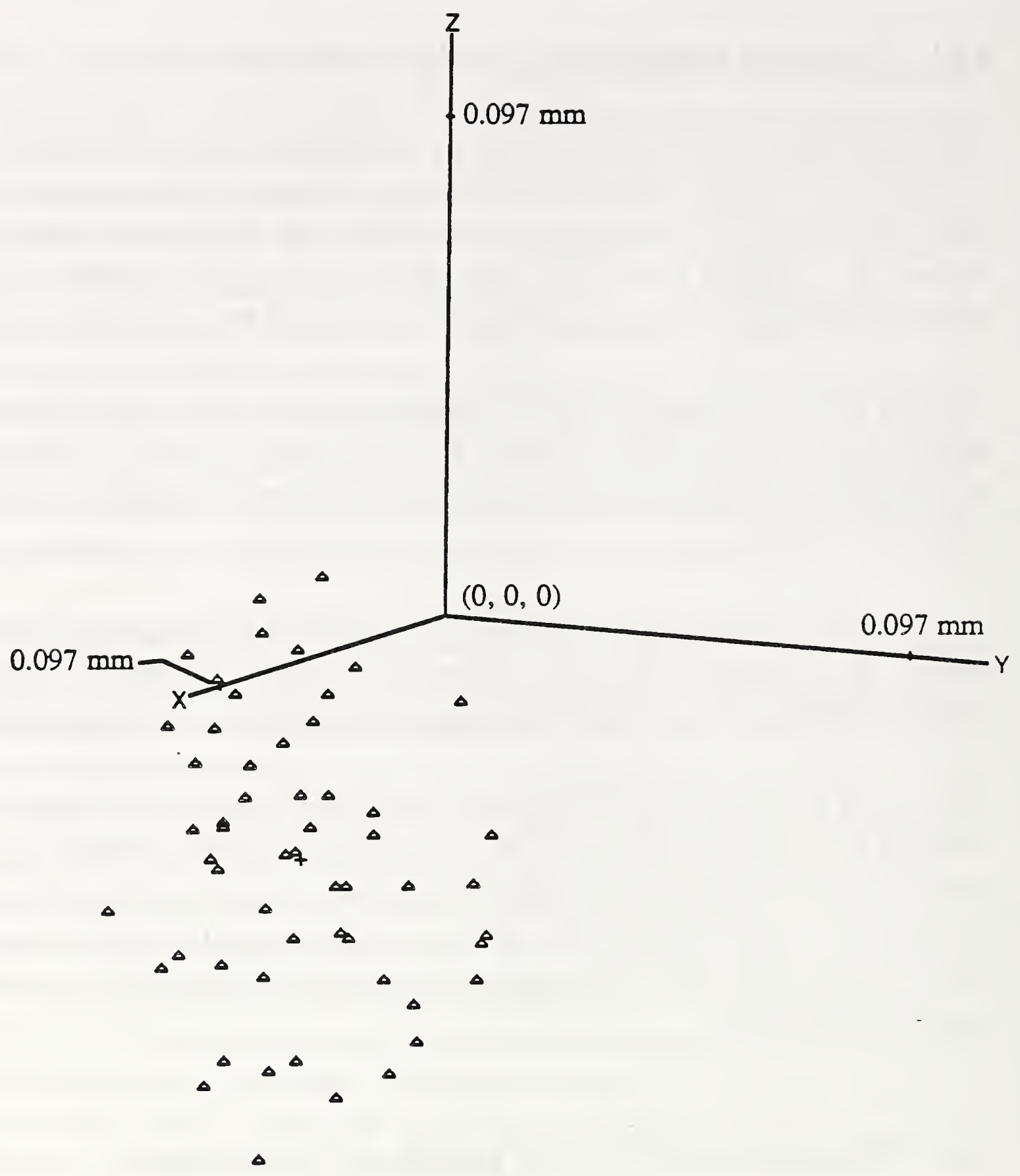

Forward Kinematics PTP Testing Errors

The cross marks the mean of the errors

Figure 4.42 Forward kinematics (continuous operation). 


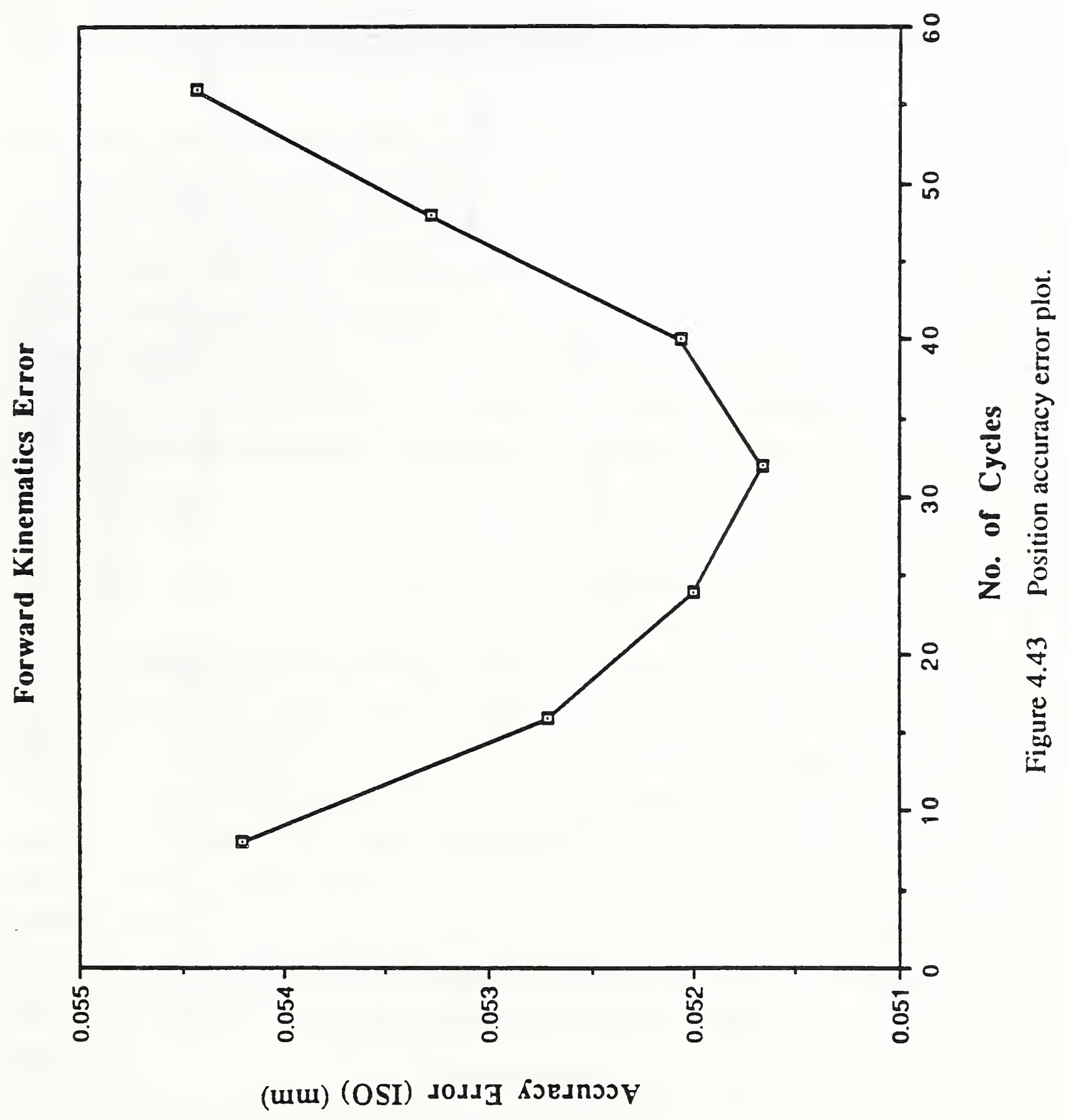




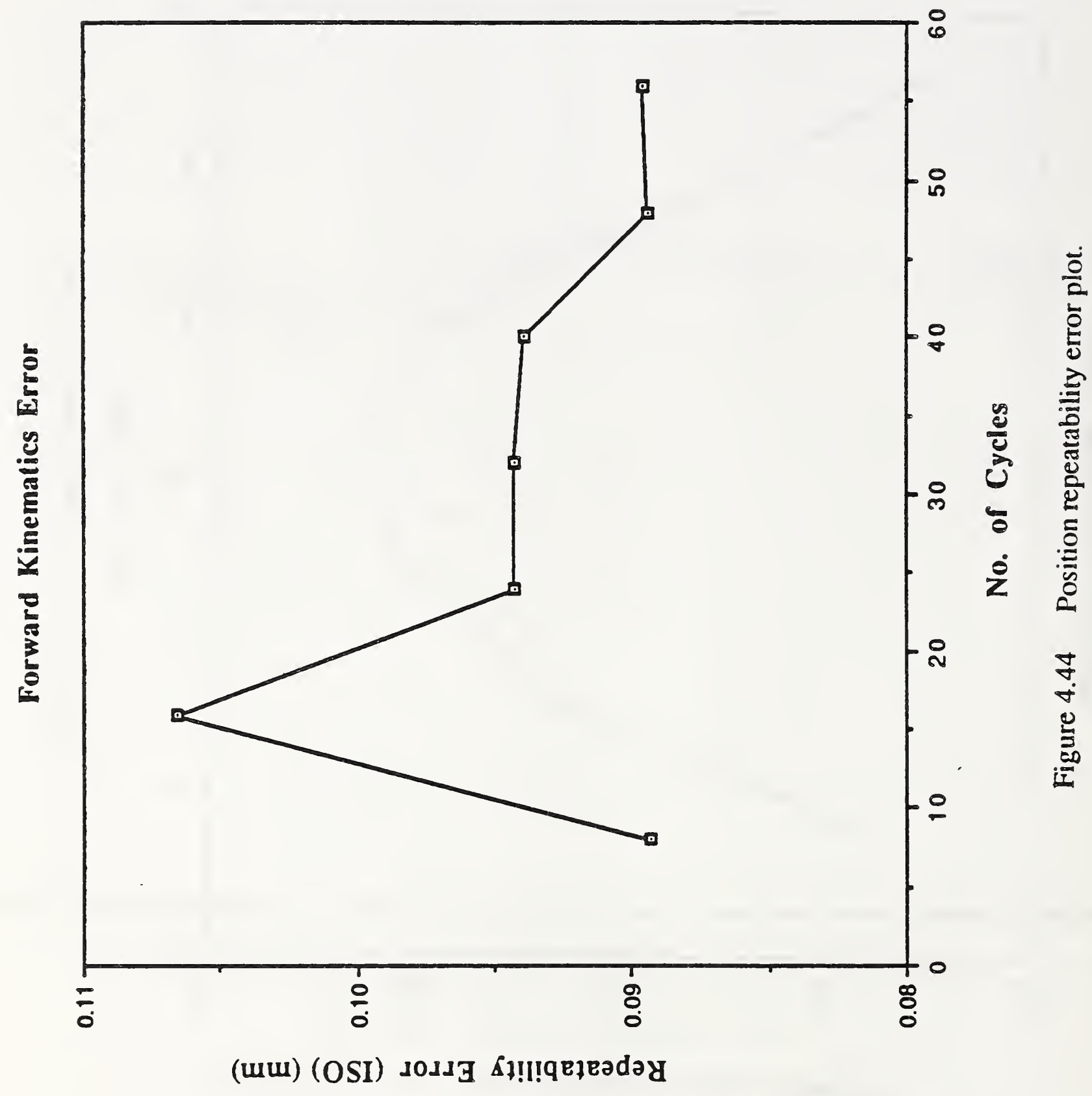


Table 4.10 in the Appendix shows the results of the analysis of the last group of data which contains all 56 measured positions. Figure 4.45 is a three dimensional plot of the calculated forward kinematics errors (triangular marks), and their mean (cross mark).

Figure 4.46 is a plot of the ISO defined accuracy error versus the number of cycles contained in each analysis group. Figure 4.47 is a plot of the ISO defined repeatability error versus the number of cycles contained in each analysis group. The variation in the values of these errors as a function of the number of cycles is rather small.

Comparing Figure 4.43 with 4.46 shows that the level of the accuracy error in the case of the interrupted operation is approximately $0.49 \mathrm{~mm}$ higher than that of the continuous operation. Comparing Figure 4.44 with 4.47 shows that the level of the repeatability error is approximately the same. This significant increase in the value of the accuracy error is probably coming mostly from the joint position initialization error. The main source of the forward kinematics errors are computer numerical algorithm errors. The random component of these type of errors has usually a very small amplitude and that explains the small variation of these errors as a function of the number of cycles.

\subsection{CONCLUSIONS}

From the variety of performance tests studied in this work it is evident that such tests can be developed for most robot operations, from conventional Point-to-Point and Continuous Path to less conventional tests like Impedance Control, etc. These performance tests can evaluate general operating modes, like off-line programming and teach mode control or be specific to a particular type of operation like assembly of truss structures, etc. Existing or proposed standard tests can be used, or new ones can be devised based on the application and the knowledge of the control procedures used. The nature of the FTS sensor nest is a significant constraint in developing and conducting these tests; still, a substantial amount of data can be gathered and significant robot performance information can be obtained from them.

The teach mode joint angles kinematics control performance test is a rather simple test to perform and still can give significant information about the quality of the position servoing capability of the robot. This mode of operation gave the lowest accuracy and repeatability 


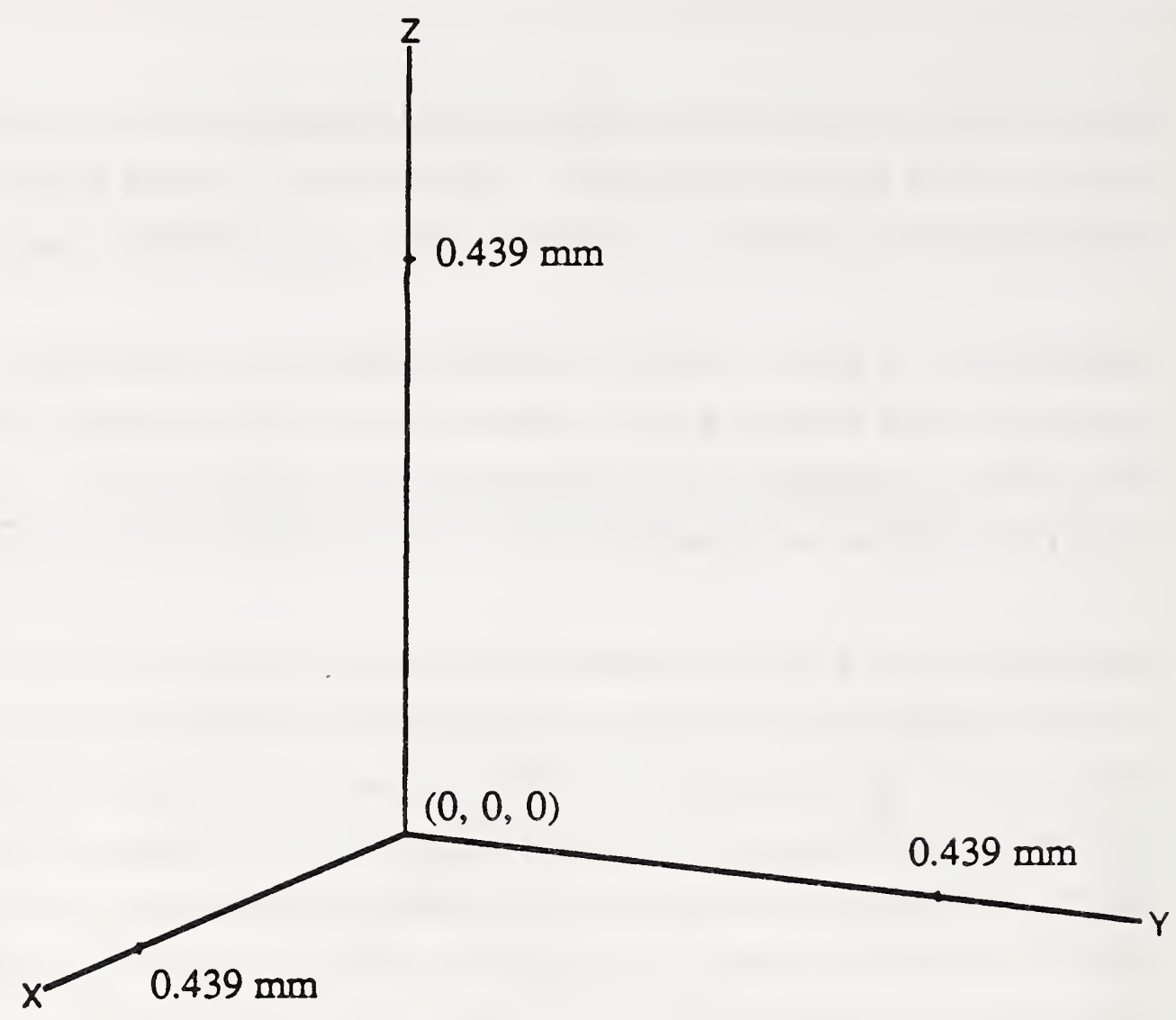

Forward Kinematics PTP Testing Errors

The cross marks the mean of the errors

Figure 4.45 Forward kinematics (interrupted operation). 


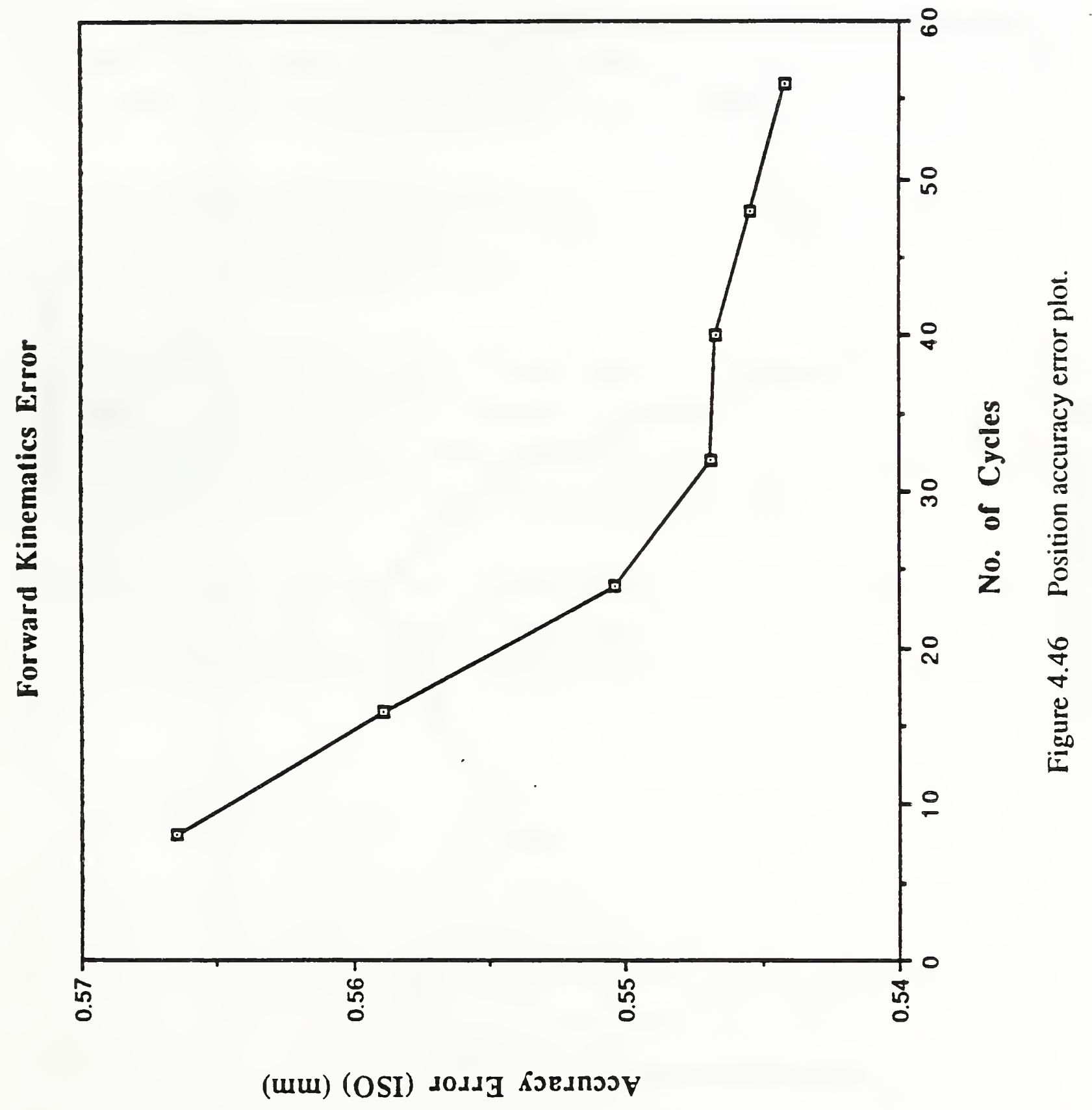




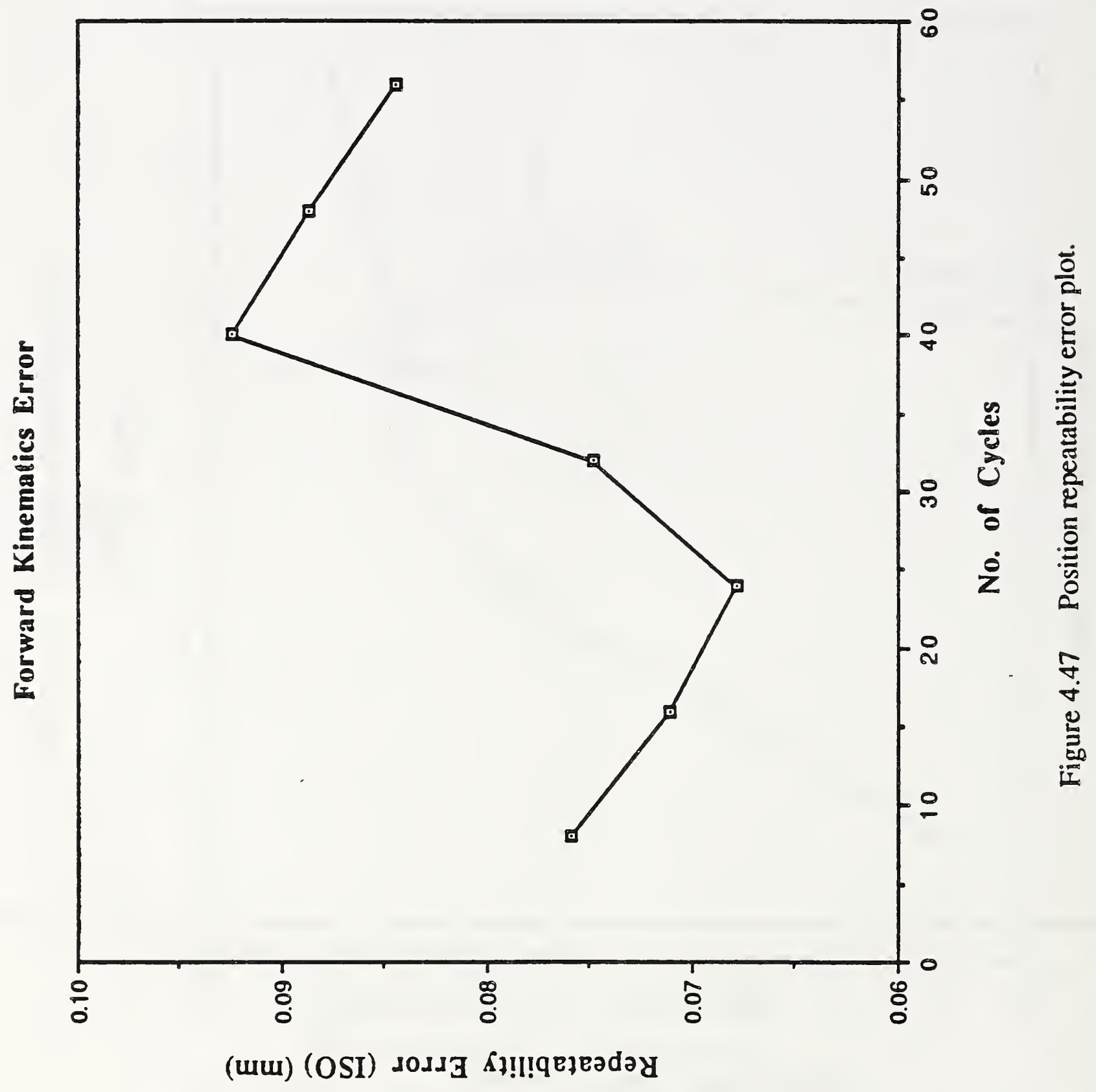


errors. The teach mode inverse kinematics control errors are higher than those of the teach mode joint angles kinematics control. Thus, Cartesian interpolated motion should only be used where the requirement for a straight line path outweighs the negative affect of additional errors. Of course, the additional errors incurred with Cartesian interpolated motion are highly dependent on the particular inverse kinematics algorithm used.

Despite the small workspace available, a coordinates transformation can be determined to convert metrology instrument measured position data to robot baseframe coordinates with reasonable accuracy. Seven data positions are sufficient to estimate the parameters of the transformation, unless the transformation will be used for non-destructive testing.

The off-line programming errors are much larger than those of the teach mode operation. Since the difference is mainly due to kinematic modelling errors special care should be given to the accuracy of kinematic calibration.

Very small incremental moves along orthogonal baseframe directions seem to be difficult to perform for the RRC K-1607 robot with the control system used. This will probably be true of most serial revolute arms. In order to characterize the robot resolution performance both the magnitude and direction of the moves should be measured.

Forward kinematics error analysis is a simple and easy to perform analysis which does not require additional testing. If possible, this analysis should be applied to position data collected before and after turning the robot power off and on in order to estimate the offset error.

All the performance plots seem to be divided into two sections depending on the number of test cycles. The first section with a number of cycles less than 8 to 16 shows high values of errors, probably due to random errors, thermal drift and dynamic motion transients. The second section with a number of cycles greater than 16 to 32 shows that the errors reach asymptotic values. Although more study is needed, it is felt that the first section characterizes robot intermittent operation, while the second is representative of prolonged continuous operation. Thus the number of test cycles used for performance testing should be decided based on the type of the robot use. In the case of the FTS this will be intermittent operations. 
Individual $\mathrm{X}, \mathrm{Y}$, and $\mathrm{Z}$-axis plots of measured achieved positions versus the number of cycles or time may reveal cyclic positioning variation. The presence of any cyclicity should be examined carefully and the peak-to-peak amplitude measured. In several instances it was observed that this amplitude was larger than the value of the ISO-defined repeatability error. Similarly any large position differences among the first few points should be measured and studied. Although further investigation would be desirable, at this time it appears that a limited motion test should not be used as a substitute for the more complex standard path positions test. As with the intermittent versus continuous operation tests above, however, the appropriateness of the limited motion test may depend on the type of robot operation being performed.

\subsection{RECOMMENDATIONS FOR FURTHER INVESTIGATION}

The prototype tests presented here have provided insight as to the suitability of a number of different metrology tests for FTS performance verification. They have also provided an. estimate of the positioning performance which may reasonably be expected from the current ICG lab manipulator/control system combination. In addition, they have indicated the relative importance of several factors which affect the test results. The test results also raise new questions, however, which would require further investigation to answer satisfactorily.

Questions regarding the performance of intermittent versus continuous operation have to be investigated further. During the first few test cycles the performance errors seem to increase significantly, probably because of thermal drift and dynamic motion transients. If a small number of cycles is used for the evaluation of these errors then random measurement errors could further increase the values of the performance errors. Two questions that need to be answered, then, are: 1) How many cycles does it take until transients and thermal drift have no effect on performance? 2) For a given number of test cycles, what percent of the measurement error is due to random measurement error?

The mechanism which results in large initial errors and cyclic variation position should be better understood. Is the integral control action associated with a time delay responsible for this type of behavior? Could the controller be modified to eliminate this problem without sacrificing the integrator? As part of this work the effect of time delays and distance of travel should be studied too. 
The sources of the distortion during small incremental moves in specific directions should be further investigated. Precision positioning requires this type of small corrective moves. What is the primary source of the error? How can the situation be improved?

The accuracy error plot of the teach mode joint angles kinematics control tests (Fig. 4.6) shows a small rise after 40 cycles. The rise is very small but perhaps it should be investigated more thoroughly. Similarly the accuracy error plot of the teach mode inverse kinematics control tests (Fig. 4.13) is different than the rest of the accuracy error plots, which resemble a simple exponential decay and perhaps should be investigated further.

\subsection{REFERENCES}

Albus, J. S., Lumia, R., 1987, "NASREM -- NASA/NBS Standard Reference Model for Telerobot Control," Goddard Conf. on Space Appl. of AI and Robotics.

API, 1989, Automated Precision Inc., Gaithersburg, Maryland, U.S.A.

Craig, J. J., 1986, Introduction to Robotics: Mechanics and Control, Addison-Wesley, Reading, MA.

Eissmann, P., 1989, Servo Interface Operator's Manual, Robotics Research Corp., Milford, Ohio, U.S.A.

Fiala, J., 1988, "Manipulator Servo Level Task Decomposition," NIST Technical Note 1255, NIST, Gaithersburg, MD.

Fiala, J., 1989a, "Note on NASREM Implementation," NIST Internal Report 89-4215, NIST, Gaithersburg, MD.

Fiala, J., 1989b, "A High-Speed Serial Interface to the Robotics Research Corporation Servo Controller," Intelligent Controls Group Internal Document.

ISO (International Organization for Standardization), 1990, "Manipulating Industrial Robots - Performance Criteria and Related Testing Methods", International Standard 9283. 
Kreutz, K., Long, M., Seraji, H., 1989, "Kinematic Functions for the 7 DOF Robotics Research Arm," NASA Conf. on Space Telerobotics, Pasadena.

Lau, K., Hocken, R., Haynes, L., 1985, "Robot performance measurements using automatic laser tracking techniques", Robotics \& Computer-Integrated Manufacturing, Vol. 2, No. 3/4, pp. 227-236.

RIA (Robotic Industries Association), 1990, "American National Standard for Industrial Robots and Robot Systems - Point-to-Point and Static Performance Characteristics Evaluation", Revision ANSI/RIA R15.05-1-1990.

Seraji, H., 1989, "Configuration Control of Redundant Manipulators: Theory and Implementation," IEEE Trans Robotics \& Automation, Vol. 4, No. 4.

Wavering, A., 1988, "Manipulator Primitive Level Task Decomposition," NIST Technical Note 1256, NIST, Gaithersburg, MD.

\subsection{ACKNOWLEDGEMENTS}

We would like to thank Mr. Chuck Giaque for preparing the drawings of the retroreflector target holder and calibration fixture and supervising its construction.

We also thank Mr. John Fiala for providing additional servo control development for the metrology tests and for assisting in the performance of the tests. 


\subsection{APPENDIX}



Table 4.1 Teach mode joint angles kinematics control analysis results.

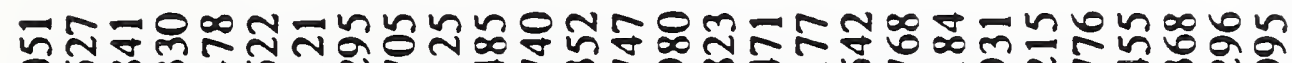
유욤

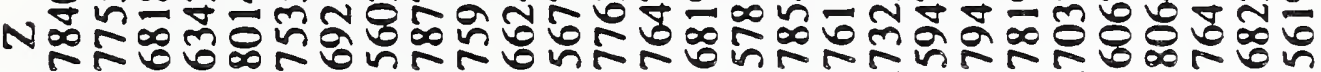

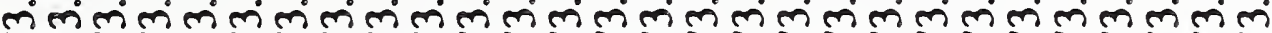

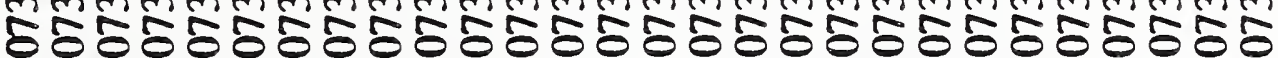

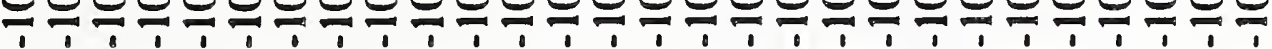

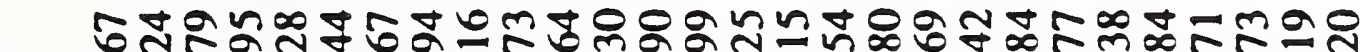

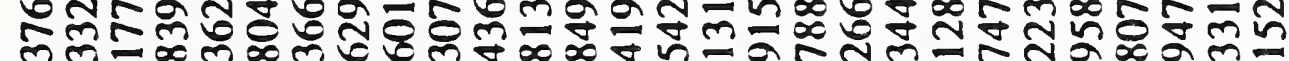
m

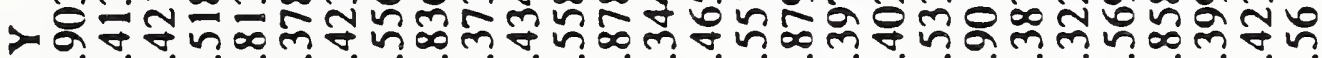

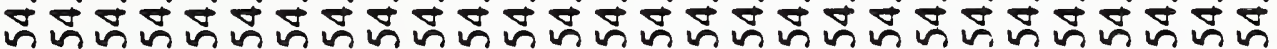

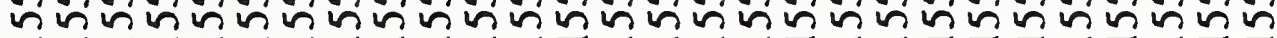

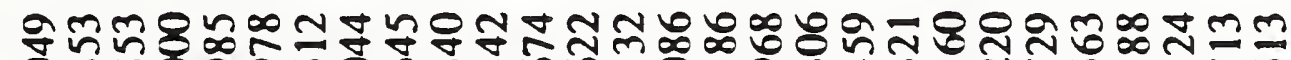

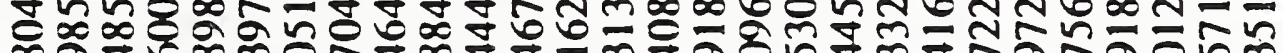

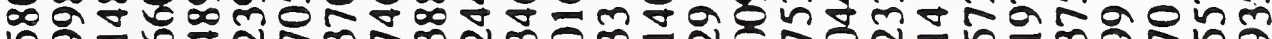

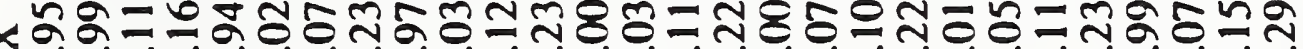
レ่

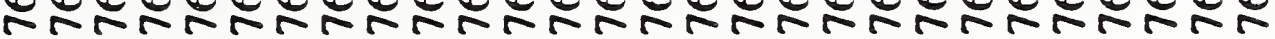

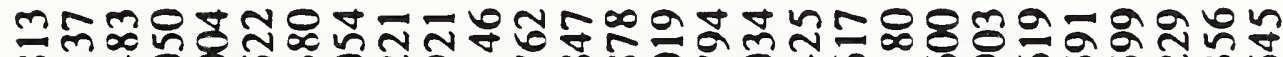
更응

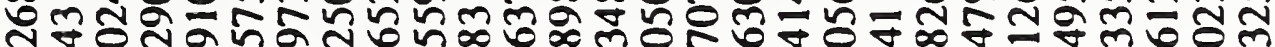

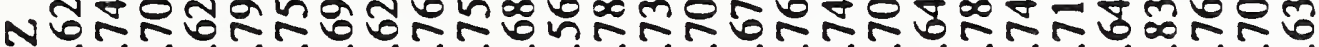

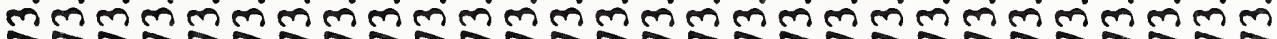

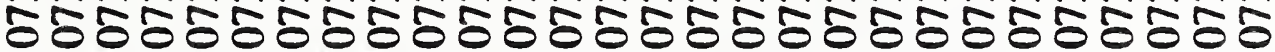

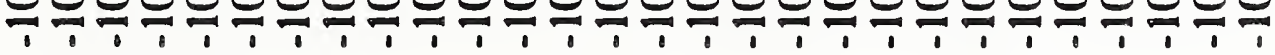

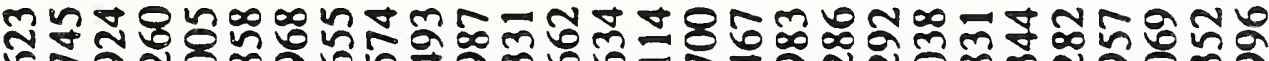

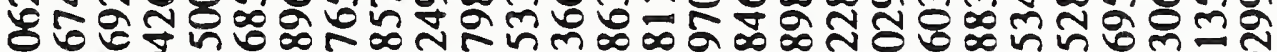

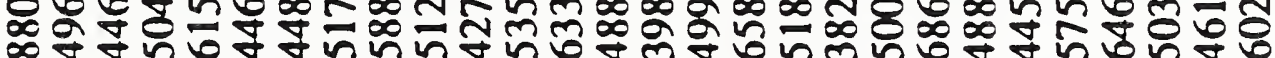

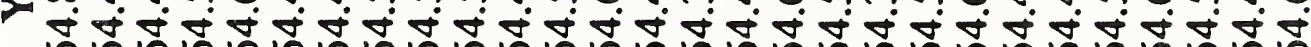

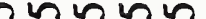

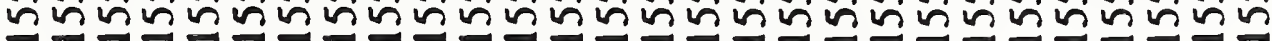

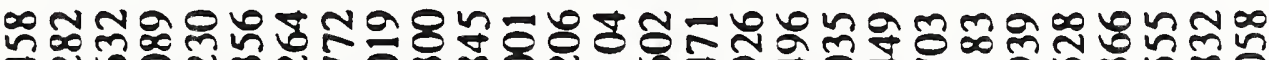
๙ $\infty$ 는

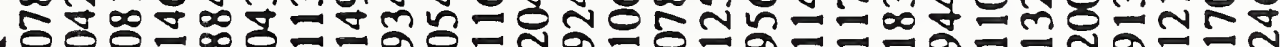

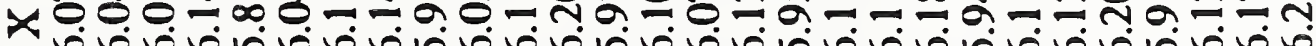
ஆ

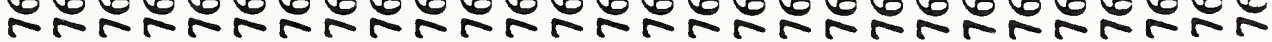


Table 4.1 Teach mode joint angles kinematics control analysis results.

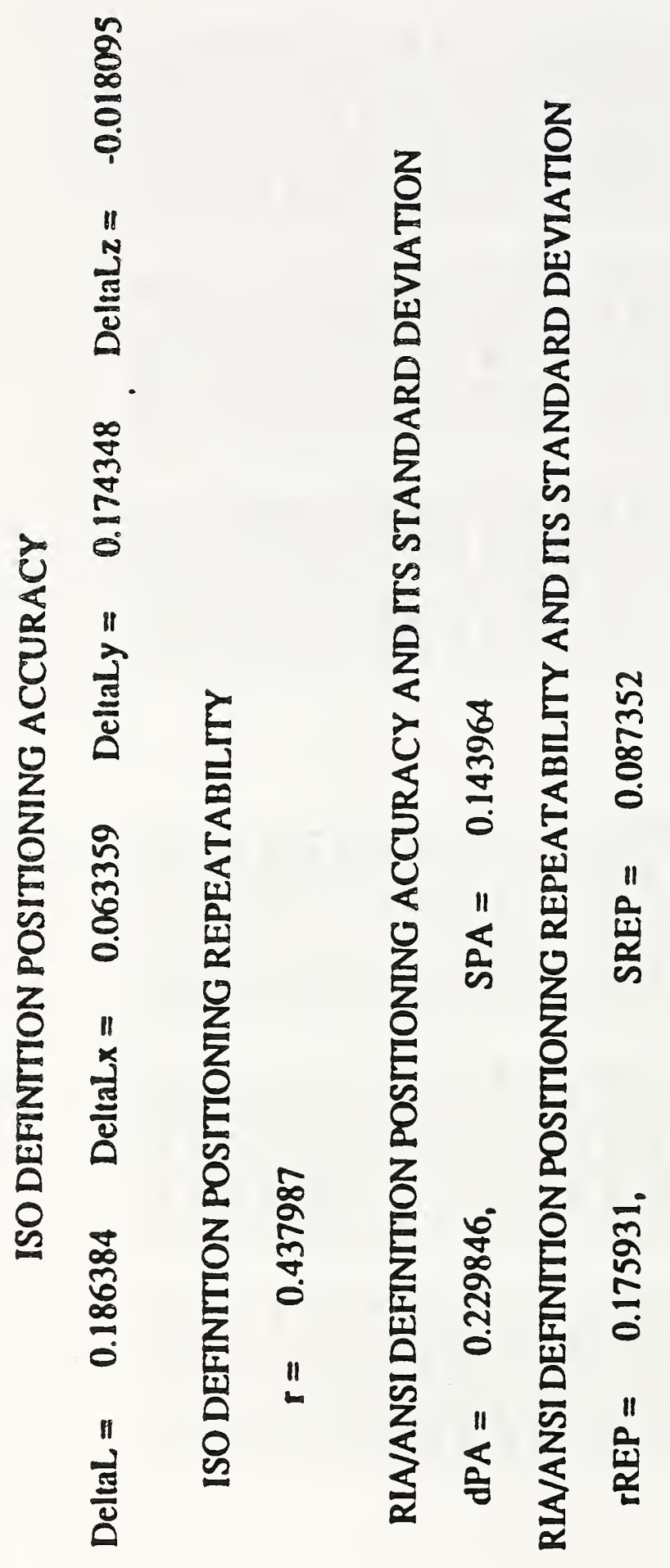


Table 4.2 Teach mode inverse kinematics control analysis results.

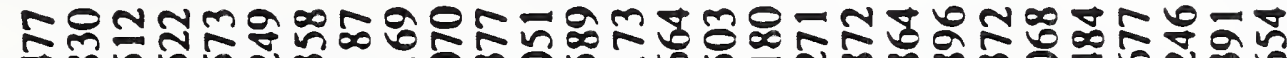
₹ซ் N

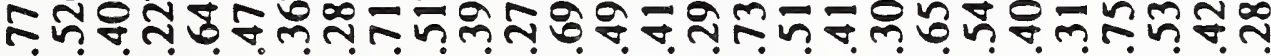

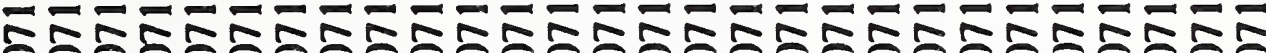
응ㅇㅇㅇㅇㅇㅇㅇㅇㅇㅇㅇㅇㅇㅇㅇㅇㅇㅇㅇㅇㅇㅇㅇㅇㅇㅇㅇㅇㅇㅇㅇㅇㅇㅇㅇㅇㅇㅇㅇㅇ

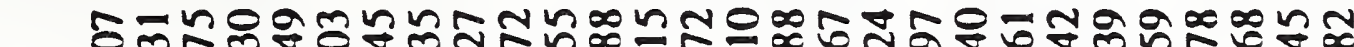

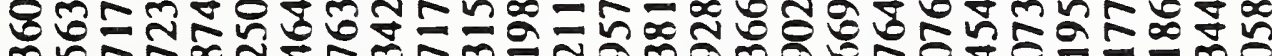
mレ5

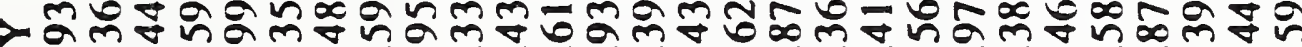

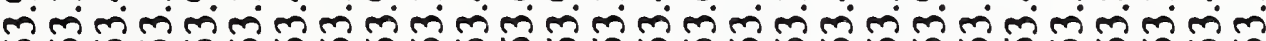

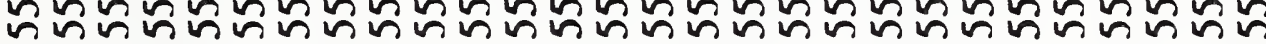

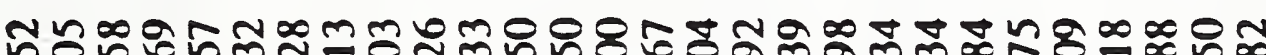

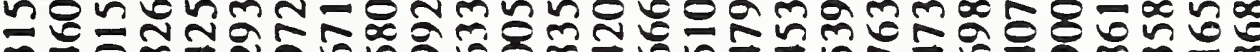
m. $x$ 궁

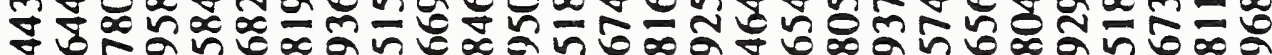

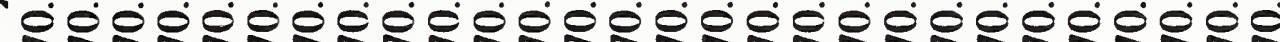

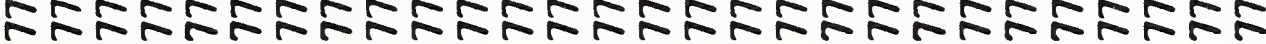

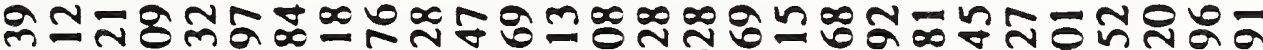

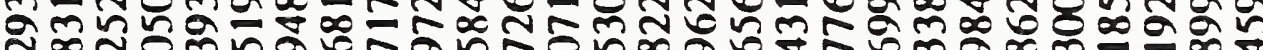
สิณ Năm

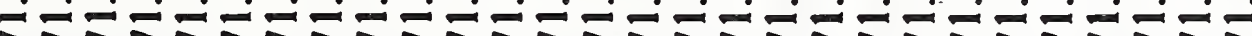

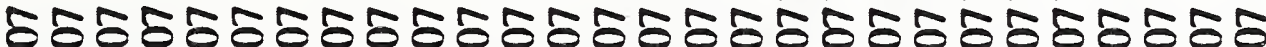

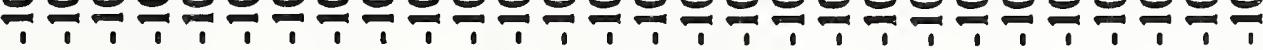

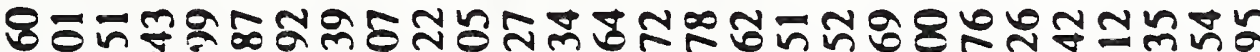

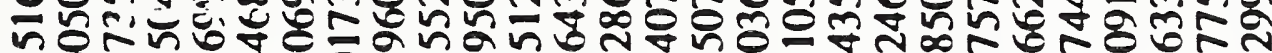

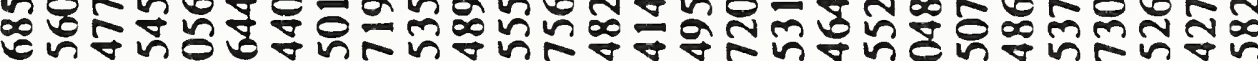

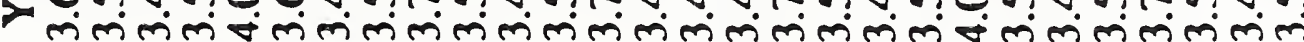

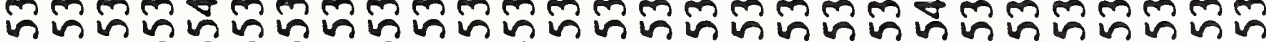
ตnต

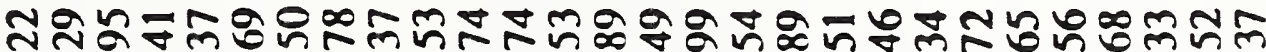
죽ำ $+\infty n n$ ก

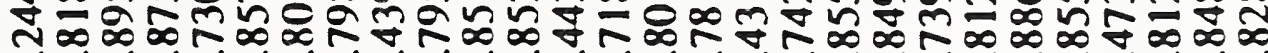

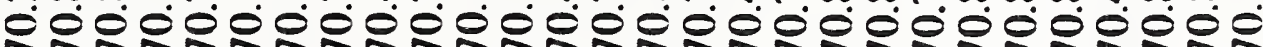

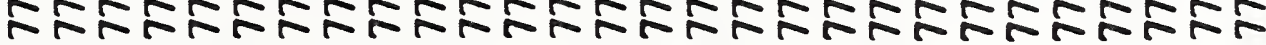


Table 4.2 Teach mode inverse kinematics control analysis results.

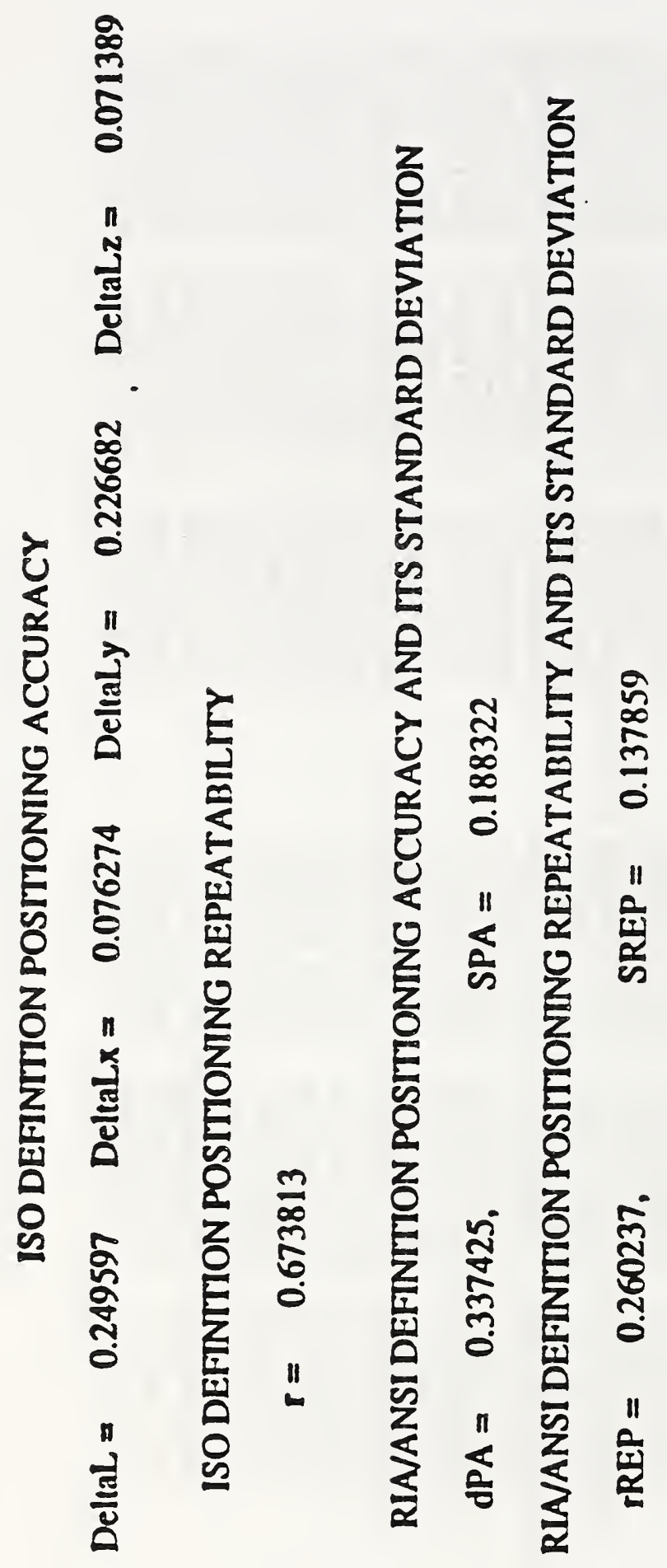


Table 4.3 Coordinates transformation analysis results (14 positions).

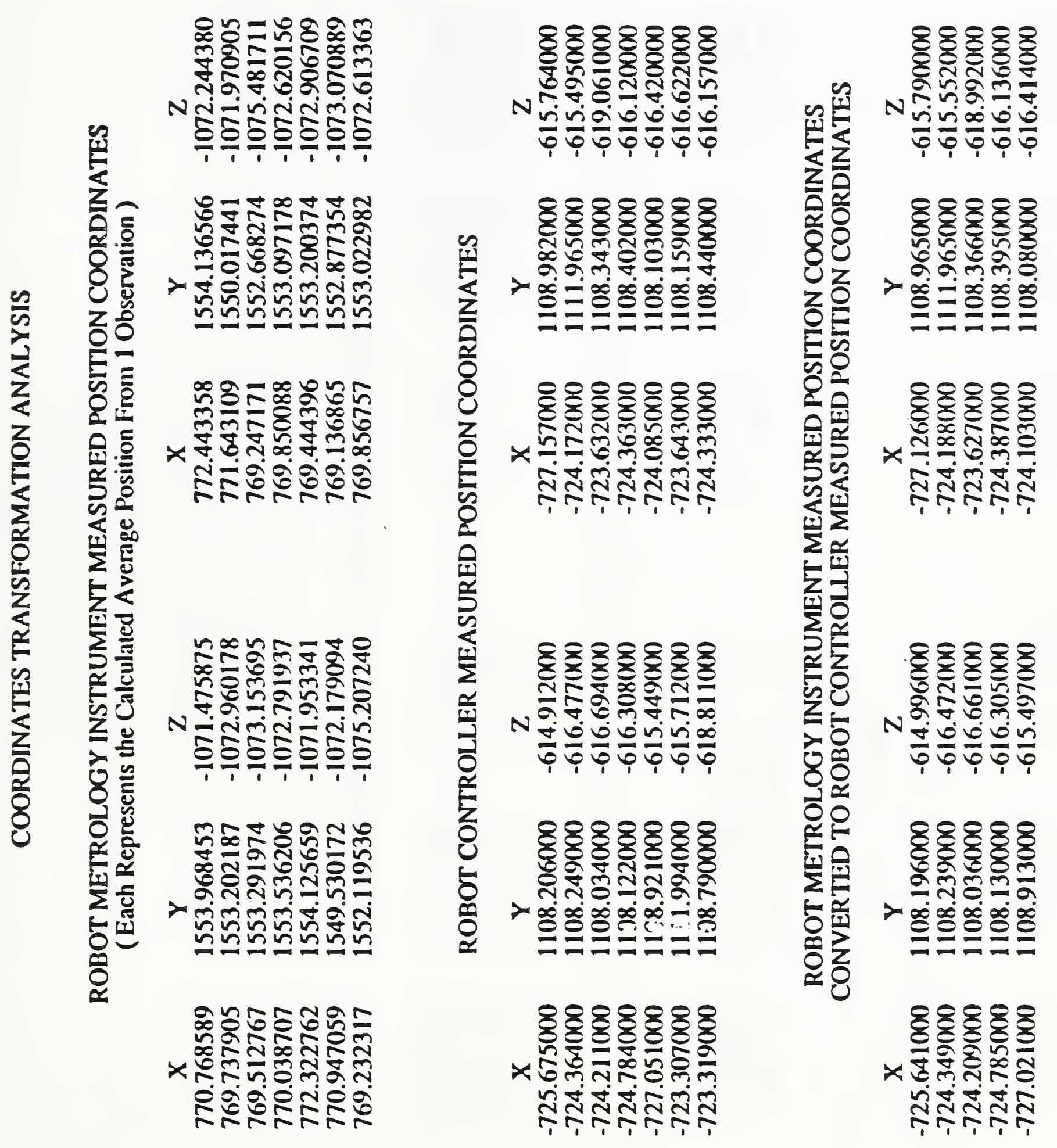


Table 4.3 Coordinates transformation analysis results (14 positions).

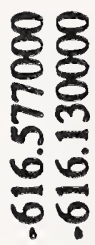

88

5

7.

$\infty$

$=$

88

을

लें

ㄷำ

$8 \delta$

nN

nio

ㅎํ

88
88
0
0
20
$=\infty$
$=0$

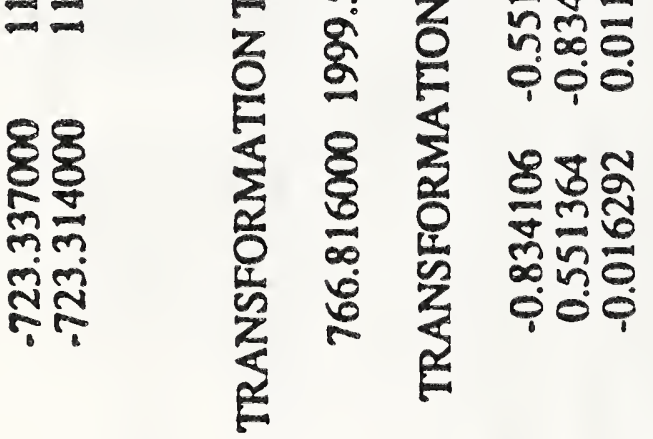


Table 4.4 Coordinates transformation analysis results (7 positions).

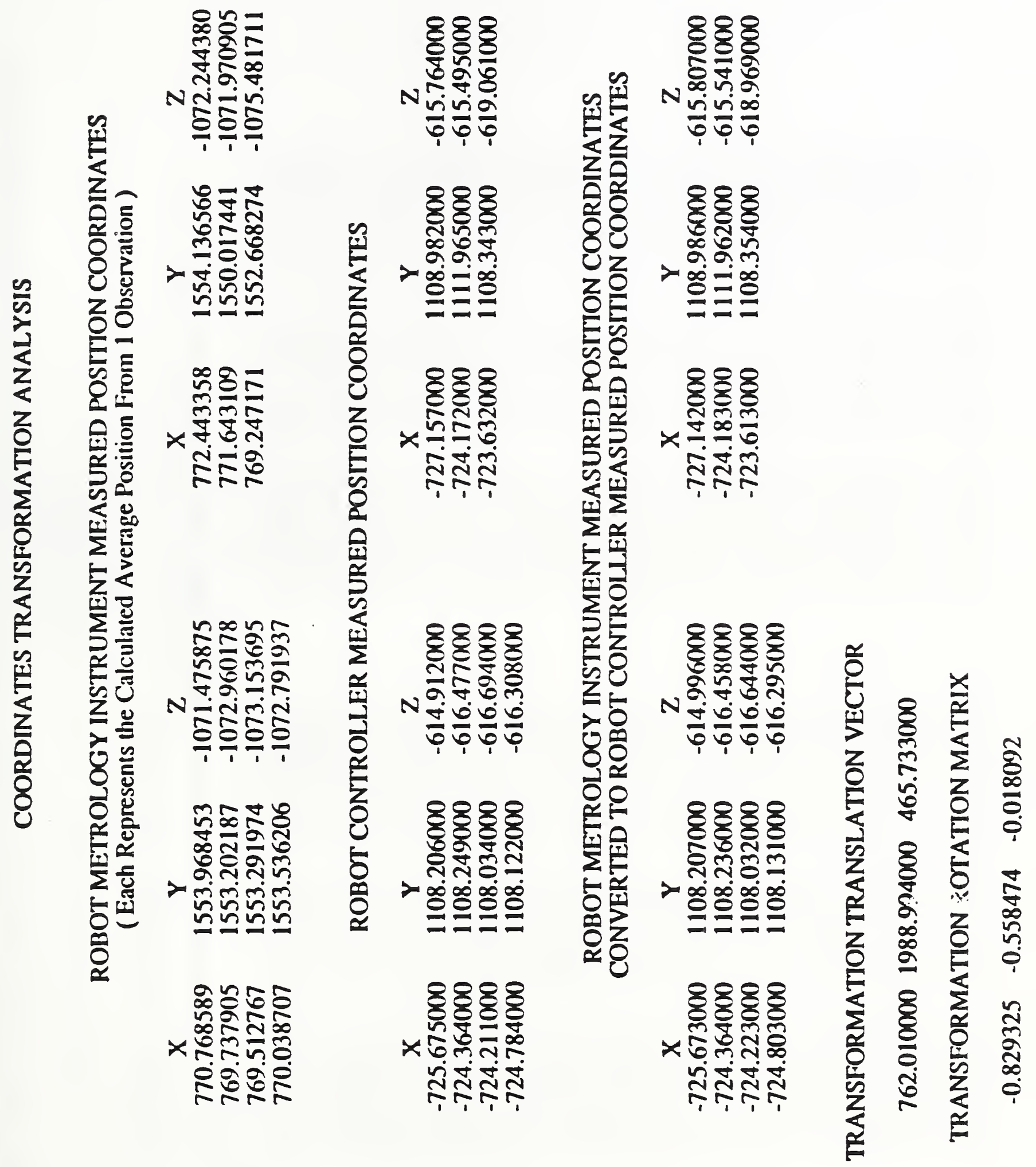


Table 4.4 Coordinates transformation analysis results ( 7 positions).

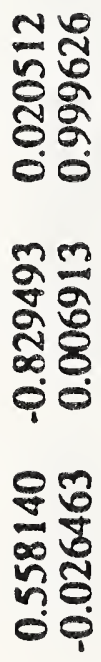


Table 4.5 Standard initial positions off-line programming analysis results.

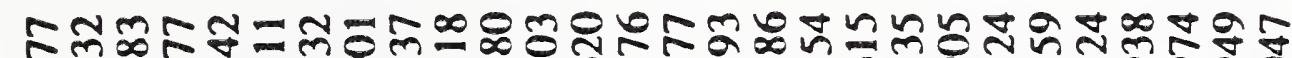

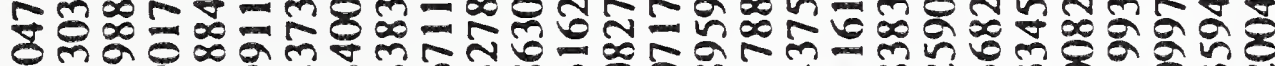

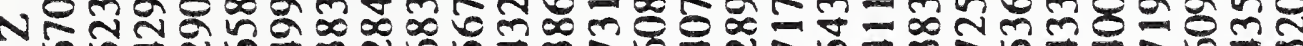
เ

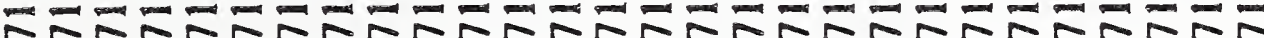

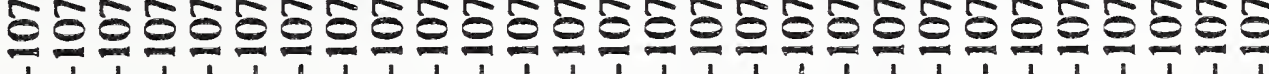

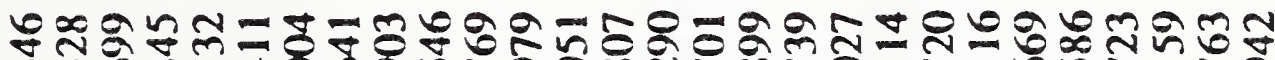

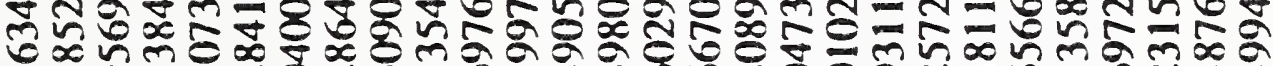

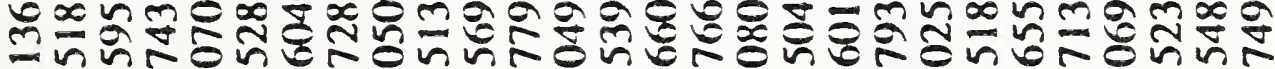
サn்

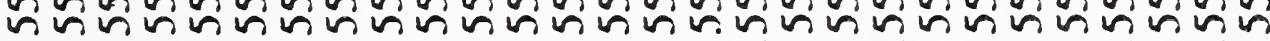

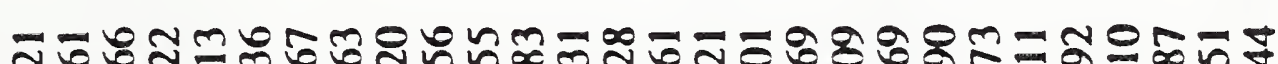

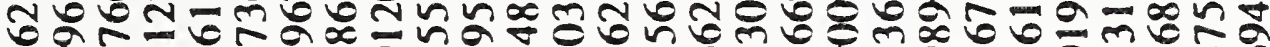

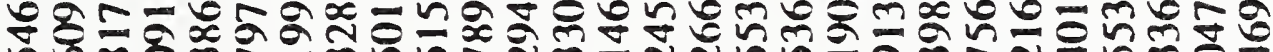
x שี

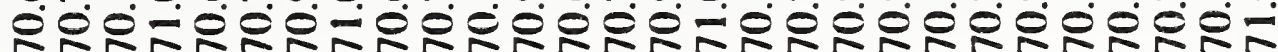

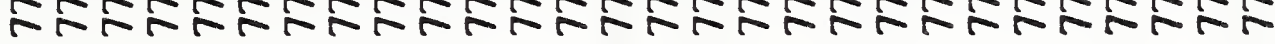

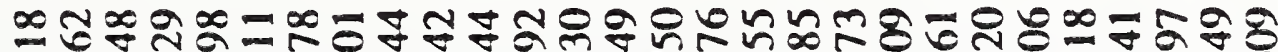

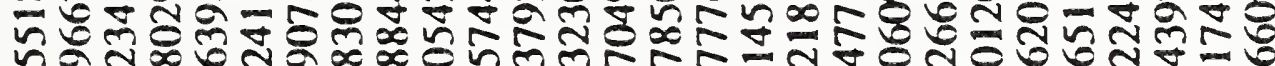

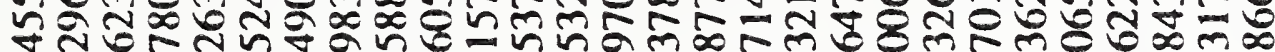
Nฟษ

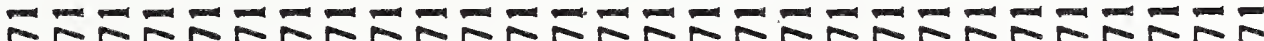

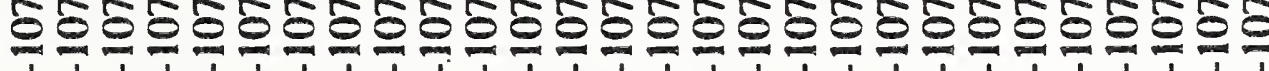

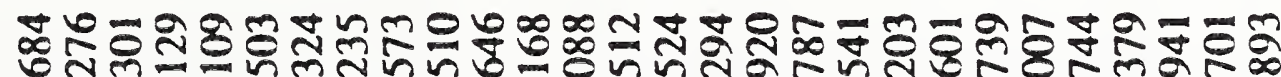
ชN

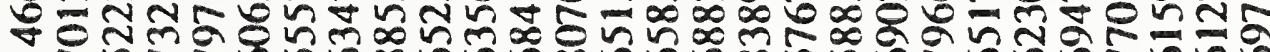

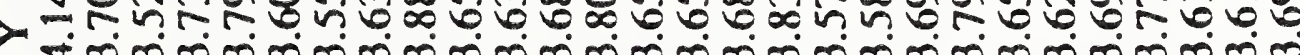

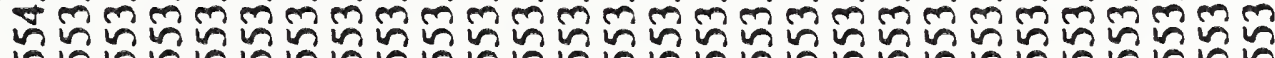

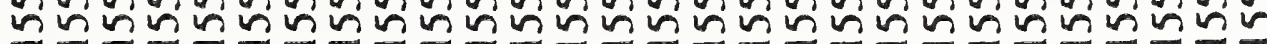

क⿻丷

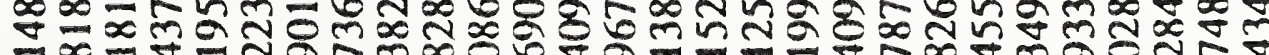

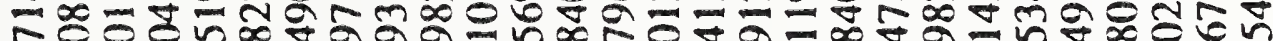

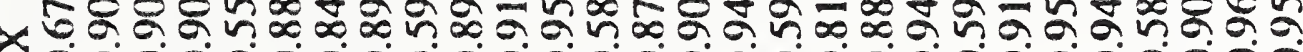
í

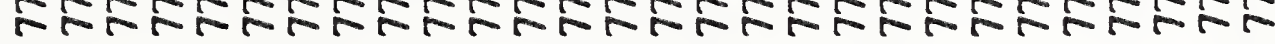


Table 4.5 Standard initial positions off-line programming analysis results.

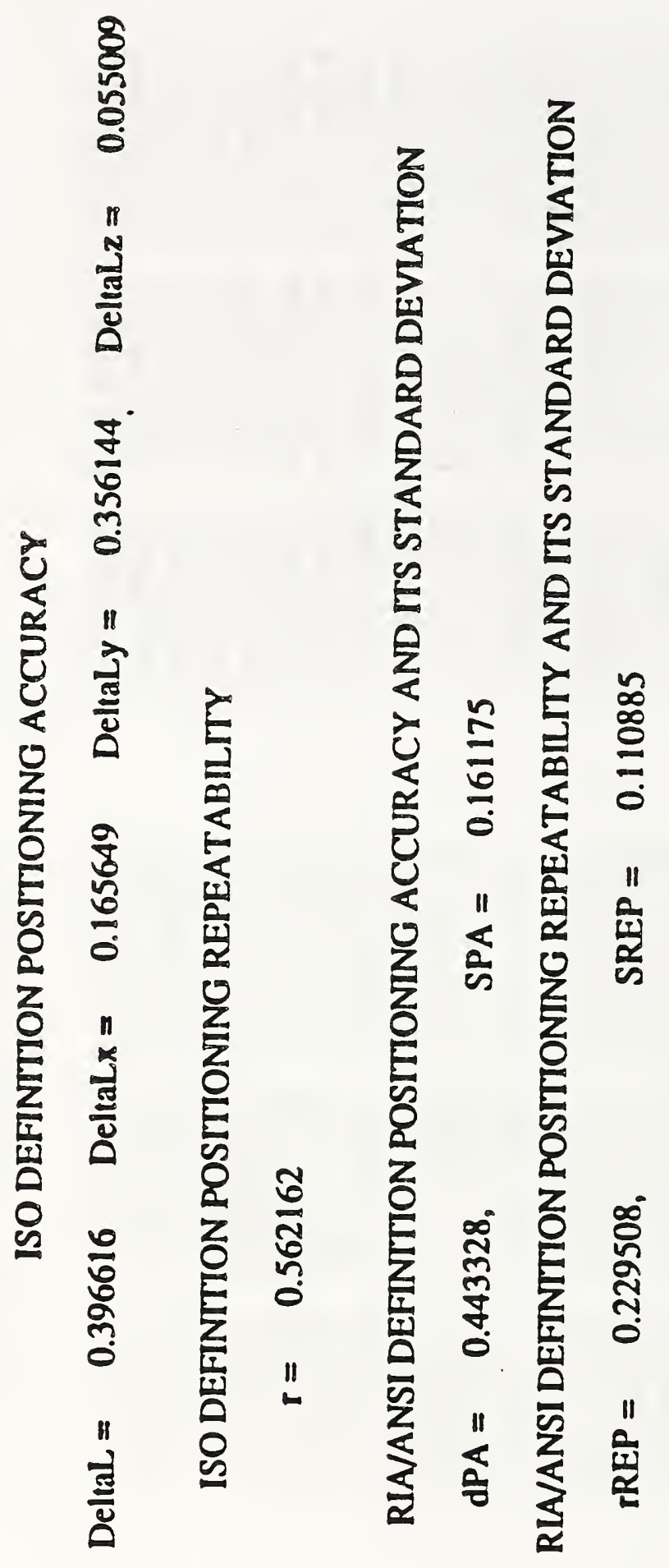


Table 4.6 Limited motion off-line programming analysis results.

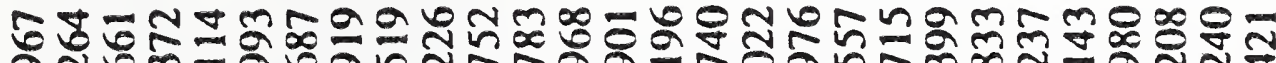

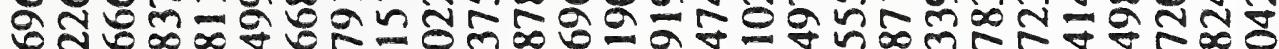

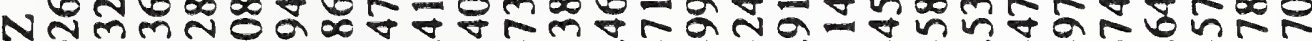

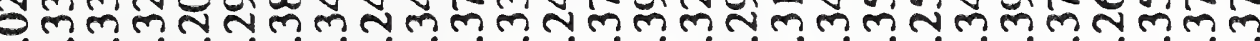

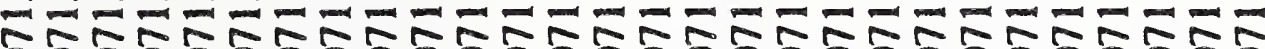

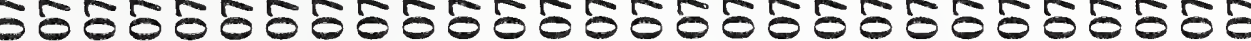
$\div \div \div \div \div \div \div \div \div \div \div \div \div \div \div \div 1 \% 1 \%$

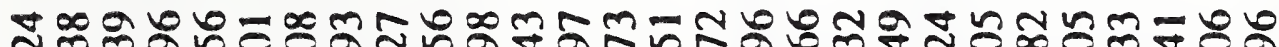
సైู은

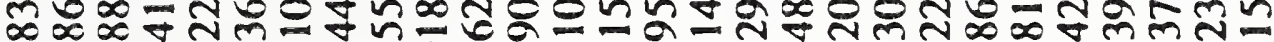
넝

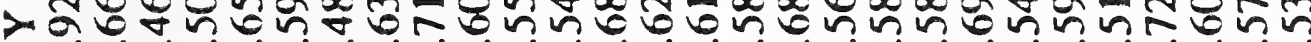

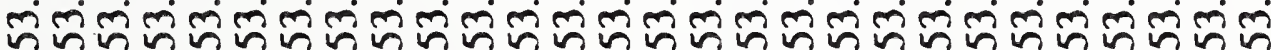

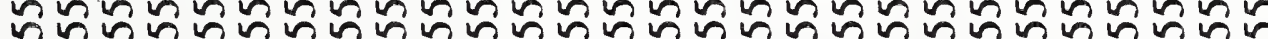

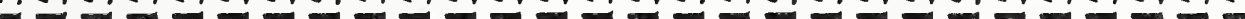

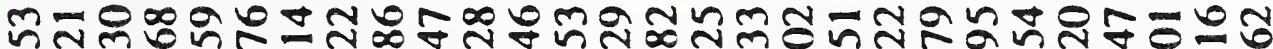

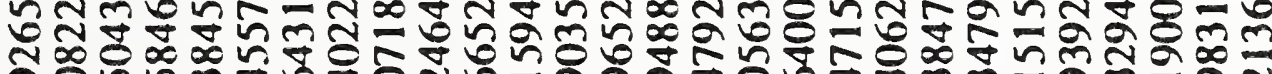
xริ유주 -

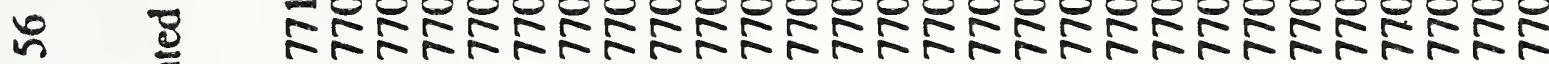

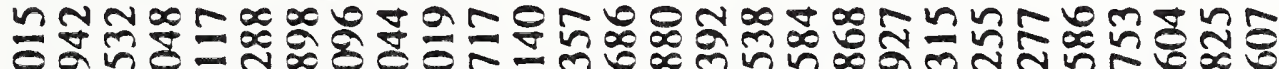

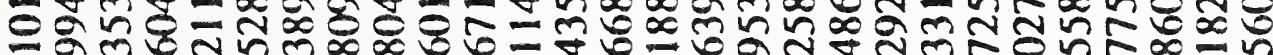

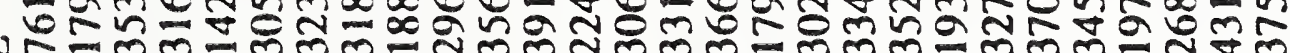

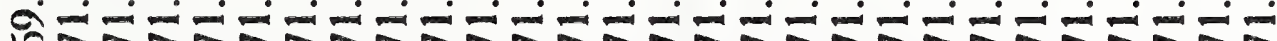

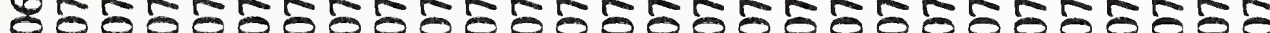

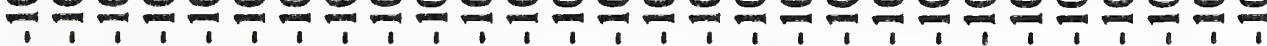

-저주ำ

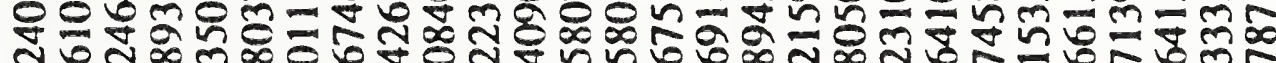
수요 \&ำ

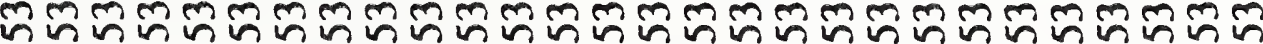

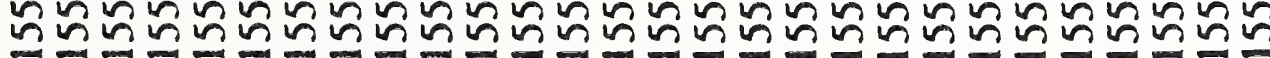

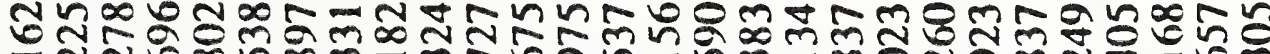
- 전

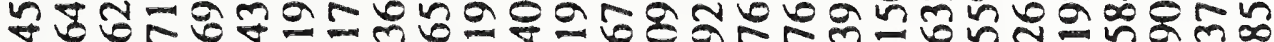
x 0 T. -

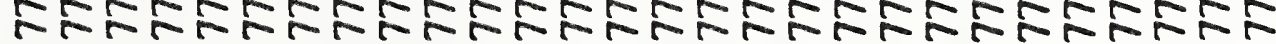


Table 4.6 Limited motion off-line programming analysis results.

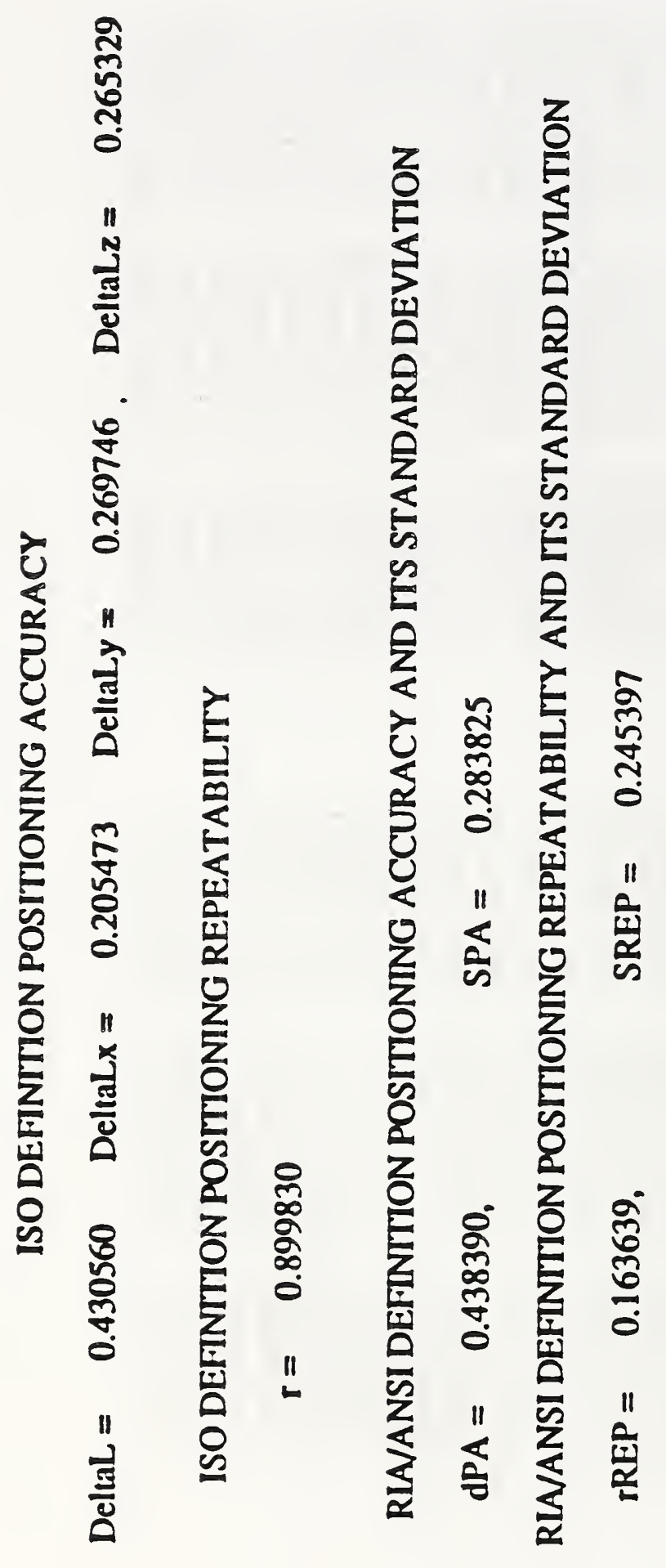


ROBOT POSITION RESOLUTION ANALYSIS

No. of incremental moves $=55$

MEASURED POSITIONS

$\begin{array}{lll}X & Y & Z\end{array}$

Movement in the X-Axis direction

$\begin{array}{lll}769.828688 & 1552.123009 & -1072.240768 \\ 770.012846 & 1552.347347 & -1072.017640 \\ 769.741931 & 1552.670747 & -1072.149516 \\ 769.609515 & 1552.928975 & -1072.218508 \\ 769.499419 & 1552.956698 & -1072.189112 \\ 769.482532 & 1552.856120 & -1072.160166 \\ 769.453115 & 1552.796014 & -1072.207286 \\ 769.439476 & 1552.732104 & -1072.234757 \\ 769.584114 & 1552.651597 & -1072.179883 \\ 769.533557 & 1552.731483 & -1072.230981 \\ 769.398620 & 1552.860820 & -1072.363012 \\ 769.304717 & 1552.907489 & -1072.436461 \\ 769.141093 & 1552.921388 & -1072.543312 \\ 769.186203 & 1552.806332 & -1072.479741 \\ 769.274922 & 1552.668315 & -1072.428045 \\ 769.373427 & 1552.593812 & -1072.371752 \\ 769.515844 & 1552.587620 & -1072.321519 \\ 769.514290 & 1552.755287 & -1072.381117 \\ 769.416616 & 1552.899018 & -1072.477586 \\ 769.291403 & 1552.997660 & -1072.573093 \\ 769.165273 & 1553.046840 & -1072.646451 \\ 769.186331 & 1552.945844 & -1072.618253 \\ 769.267106 & 1552.860580 & -1072.554508 \\ 769.343767 & 1552.767194 & -1072.489527 \\ 769.530598 & 1552.677238 & -1072.376896 \\ 769.487515 & 1552.751413 & -1072.410448 \\ 769.362265 & 1552.920291 & -1072.537185 \\ 769.268833 & 1552.989916 & -1072.600192 \\ 769.149583 & 1553.019984 & -1072.679752 \\ 769.192606 & 1552.935061 & -1072.641090 \\ 769.270564 & 1552.842614 & -1072.566315 \\ 769.380349 & 1552.737912 & -1072.487641 \\ 769.546438 & 1552.648958 & -1072.367089 \\ 769.514448 & 1552.726733 & -1072.417537 \\ 769.403209 & 1552.873356 & -1072.514440 \\ 769.317809 & 1552.949127 & -1072.568207 \\ 769.199455 & 1552.982046 & -1072.638194 \\ 769.213539 & 1552.907020 & -1072.615572 \\ 769.291861 & 1552.817134 & -1072.555462 \\ 769.365641 & 1552.749103 & -1072.489806 \\ 769.526753 & 1552.663010 & -1072.404463 \\ 769.497855 & 1552.749564 & -1072.438777 \\ 769.402814 & 1552.867680 & -1072.517230 \\ 769.299292 & 1552.954236 & -1072.584011\end{array}$

Table 4.7 Robot position resolution analysis results (commanded incremental moves of $0.15 \mathrm{~mm}$ ). 
769.209451

769.246736

769.287038

769.368220

769.569030

769.569296

769.448119

769.379350

769.247404

769.245821

769.341893

769.411202
1552.946267 1552.875085 1552.810661 1552.722823 1552.670203 1552.725478 1552.875181 1552.954982 1552.974879 1552.920193 1552.813947 1552.725134
$-1072.656674$ $-1072.603428$ $-1072.544846$ $-1072.483016$ $-1072.363411$ $-1072.384205$ $-1072.487997$ $-1072.541138$ $-1072.624706$ $-1072.596496$ $-1072.517878$ $-1072.462664$

Movement in the Y-Axis direction

\begin{tabular}{|c|c|c|}
\hline $\begin{array}{l}44106 \\
89685 \\
43817 \\
00221 \\
25016 \\
85076 \\
46207 \\
78523 \\
51282 \\
08669 \\
89324 \\
94249\end{array}$ & $\begin{array}{l}1552.786055 \\
1552.652785 \\
1552.530691 \\
1552.461085 \\
1552.423168 \\
1552.531586 \\
1552.671252 \\
1552.860272 \\
1553.017973 \\
1552.897746 \\
1552.759357 \\
1552.595639 \\
1552.422266 \\
1552.489055 \\
1552.623411 \\
1552.775439 \\
1552.961474 \\
1552.851164 \\
1552.702293 \\
1552.559834 \\
1552.497469 \\
1552.592083 \\
1552.757798 \\
1552.907815 \\
1553.091674 \\
1553.010469 \\
1552.835546 \\
1552.659555 \\
1552.508125 \\
1552.571180 \\
1552.713770 \\
1552.849155 \\
1552.987431 \\
1552.926359 \\
1552.780944 \\
1552.612237 \\
1552.456541 \\
1555525830\end{array}$ & $\begin{array}{l}-1072.387552 \\
-1072.291611 \\
-1072.230492 \\
-1072.252513 \\
-1072.277282 \\
-1072.399886 \\
-1072.520693 \\
-1072.679352 \\
-1072.817439 \\
-1072.701608 \\
-1072.577447 \\
-1072.453135 \\
-1072.330650 \\
-1072.406199 \\
-1072.517640 \\
-1072.648589 \\
-1072.775819 \\
-1072.671856 \\
-1072.535210 \\
-1072.412861 \\
-1072.310282 \\
-1072.419420 \\
-1072.540917 \\
-1072.653669 \\
-1072.793672 \\
-1072.716252 \\
-1072.550536 \\
-1072.413572 \\
-1072.338230 \\
-1072.403442 \\
-1072.497592 \\
-1072.630774 \\
-1072.724870 \\
-1072.633883 \\
-1072.532363 \\
-1072.404313 \\
-1072.298467 \\
-1072.374886\end{array}$ \\
\hline
\end{tabular}




$\begin{array}{lll}769.656425 & 1552.762674 & -1072.585300 \\ 769.479090 & 1552.907238 & -1072.727502 \\ 769.552307 & 1552.830785 & -1072.646475 \\ 769.658991 & 1552.692456 & -1072.530593 \\ 769.816772 & 1552.526042 & -1072.408142 \\ 769.909129 & 1552.416012 & -1072.333371 \\ 769.848400 & 1552.474677 & -1072.397265 \\ 769.731534 & 1552.586166 & -1072.501032 \\ 769.611098 & 1552.721467 & -1072.608937 \\ 769.445826 & 1552.903986 & -1072.758775 \\ 769.532323 & 1552.833166 & -1072.667886 \\ 769.676123 & 1552.663666 & -1072.524202 \\ 769.797027 & 1552.493368 & -1072.404380 \\ 769.911406 & 1552.374403 & -1072.309295 \\ 769.865877 & 1552.434522 & -1072.377612 \\ 769.728256 & 1552.576002 & -1072.474208 \\ 769.591258 & 1552.718960 & -1072.602752\end{array}$

Movement in the Z-Axis direction

\begin{tabular}{|c|c|c|}
\hline $\begin{array}{l}9.464747 \\
9.456738 \\
9.532865 \\
9.521339 \\
9.463507 \\
9.404349 \\
9.423666 \\
9.419958 \\
9.461396 \\
9.443185 \\
9.520245 \\
9.505617 \\
9.458865 \\
9.411082 \\
9.434586 \\
9.457342 \\
9.459966 \\
9.489168 \\
9.557342 \\
9.546264 \\
9.517216 \\
9.444782 \\
9.446615\end{array}$ & $\begin{array}{l}1552.919976 \\
1552.912704 \\
1552.955548 \\
1552.973582 \\
1552.996548 \\
1552.941817 \\
1552.865209 \\
1552.829557 \\
1552.755819 \\
1552.759626 \\
1552.831741 \\
1552.902866 \\
1552.929203 \\
1552.904981 \\
1552.853113 \\
1552.838722 \\
1552.735936 \\
1552.766842 \\
1552.863041 \\
1552.933367 \\
1552.974137 \\
1552.950880 \\
1552.904166 \\
1552.839364 \\
1552.758639 \\
1552.808036 \\
1552.879178 \\
1552.963723 \\
1552.990513 \\
1552.975444 \\
1552.910917 \\
1552.836604 \\
1552.767582\end{array}$ & $\begin{array}{l}82401 \\
26139 \\
31459 \\
35314 \\
56795 \\
55645 \\
89231 \\
70476 \\
96225 \\
73227 \\
83728 \\
11924 \\
39020 \\
33026 \\
58362 \\
46732 \\
99388 \\
89078 \\
78920 \\
92430 \\
93954 \\
26891 \\
46342 \\
57068 \\
95905 \\
84694 \\
59680 \\
45074 \\
75575\end{array}$ \\
\hline
\end{tabular}

Table 4.7 Robot position resolution analysis results (commanded incremental moves of $0.15 \mathrm{~mm}$ ). 


769.539407
769.478738
769.485672
769.484718
769.499071
769.544371
769.596184
769.584502
769.531099
769.489331
769.471603
769.476364
769.524465
769.537640
769.579964
769.592567
769.550440
769.496166
769.491034
769.491565
769.498711
769.543929

15

$1552.996060-1072.349014$

$1553.082875-1072.310918$

$1553.066398-1072.306300$

$1553.029320-1072.315422$

$1552.953509-1072.295583$

$1552.855515-1072.318826$

$1552.891079-1072.320811$

$1552.975657-1072.356023$

$1553.056010-1072.364341$

$1553.105682-1072.315345$

$1553.066330-1072.313296$

$1553.011374-1072.293721$

$1552.964339-1072.294652$

$1552.862863-1072.312309$

$1552.890475-1072.326722$

$1552.951797-1072.343921$

$1553.039819-1072.363942$

$1553.077694-1072.301344$

$1553.053668-1072.303886$

$1553.010178-1072.297397$

$1552.938461-1072.286121$
Table 4.7 Robot position resolution analysis results (commanded incremental moves of $0.15 \mathrm{~mm}$ ).

MEAN VALUE AND STANDARD DEVIATION OF THE RESOLUTION IN THE X-AXIS DIRECTION

Resolution $=0.156625$ Sigma $=0.070600$

MEAN VALUE AND STANDARD DEVIATION OF THE RESOLUTION IN THE Y-AXIS DIRECTION

Resolution $=0.208025$ Sigma $=0.056606$

MEAN VALUE AND STANDARD DEVIATION OF THE RESOLUTION IN THE Z-AXIS DIRECTION

Resolution $=0.073010$ Sigma $=0.031027$ 
ROBOT POSIIION RESOLUTION ANALYSIS

No. of incremental moves $=55$

MEASURED POSITIONS

$\begin{array}{lll}X & Y\end{array}$

Movement in the X-Axis direction

$\begin{array}{lll}769.767736 & 1552.677351 & -1072.318677 \\ 769.959476 & 1552.493000 & -1072.404938 \\ 770.458953 & 1553.186239 & -1072.681214 \\ 770.941056 & 1553.311439 & -1072.497534 \\ 771.412926 & 1553.387432 & -1072.315250 \\ 771.259507 & 1553.816508 & -1072.530830 \\ 770.719725 & 1553.808064 & -1072.875978 \\ 770.117304 & 1553.668466 & -1073.128186 \\ 769.538943 & 1552.967463 & -1072.955386 \\ 769.701115 & 1552.685883 & -1072.768096 \\ 770.214106 & 1552.822927 & -1072.642508 \\ 770.735293 & 1553.075107 & -1072.576545 \\ 771.147331 & 1553.165604 & -1072.405544 \\ 770.895156 & 1553.512859 & -1072.674313 \\ 770.470043 & 1553.702783 & -1072.952462 \\ 769.912160 & 1553.453461 & -1073.189525 \\ 769.375701 & 1552.845273 & -1072.976701 \\ 769.579580 & 1552.468667 & -1072.799365 \\ 770.138143 & 1552.688213 & -1072.582710 \\ 770.612609 & 1553.034584 & -1072.622331 \\ 771.120362 & 1553.157121 & -1072.412558 \\ 770.962985 & 1553.466903 & -1072.605501 \\ 770.593878 & 1553.690057 & -1072.841959 \\ 769.999646 & 1553.425112 & -1073.105567 \\ 769.548983 & 1552.780151 & -1072.885067 \\ 769.708927 & 1552.445798 & -1072.699086 \\ 770.249906 & 1552.608840 & -1072.465829 \\ 770.704245 & 1552.995287 & -1072.546031 \\ 771.245940 & 1553.153799 & -1072.290364 \\ 771.090819 & 1553.452655 & -1072.483109 \\ 770.692832 & 1553.698073 & -1072.758666 \\ 770.053177 & 1553.447086 & -1073.075927 \\ 769.474686 & 1552.662117 & -1072.887676 \\ 769.627103 & 1552.365088 & -1072.740549 \\ 770.120102 & 1552.563536 & -1072.523310 \\ 770.680202 & 1552.876266 & -1072.525446 \\ 771.206906 & 1553.041724 & -1072.263285 \\ 770.983367 & 1553.426319 & -1072.531727 \\ 770.576256 & 1553.657190 & -1072.822223 \\ 769.924041 & 1553.365318 & -1073.130337 \\ 769.411359 & 1552.627876 & -1072.920133 \\ 769.571407 & 1552.344407 & -1072.765352 \\ 770.080213 & 1552.547627 & -1072.551831 \\ 770.549522 & 1552.903774 & -1072.604166\end{array}$

Table 4.8 Robot position resolution analysis results (commanded incremental moves of $0.5 \mathrm{~mm}$ ). 


$\begin{array}{lll}771.151284 & 1553.042528 & -1072.332503 \\ 770.999096 & 1553.386752 & -1072.491405 \\ 770.622142 & 1553.617018 & -1072.780980 \\ 769.972138 & 1553.350320 & -1073.099202 \\ 769.453392 & 1552.678852 & -1072.921105 \\ 769.595449 & 1552.356478 & -1072.770007 \\ 770.120285 & 1552.518938 & -1072.534224 \\ 770.585367 & 1552.905407 & -1072.586575 \\ 771.157040 & 1553.053931 & -1072.323580 \\ 771.004052 & 1553.364396 & -1072.491347 \\ 770.633659 & 1553.624181 & -1072.746394 \\ 770.006714 & 1553.363175 & -1073.073219\end{array}$

Movement in the Y-Axis direction

$\begin{array}{lll}769.382215 & 1552.382531 & -1072.837471 \\ 769.670122 & 1551.785465 & -1072.520563 \\ 769.985904 & 1551.085509 & -1072.308480 \\ 770.392957 & 1550.835708 & -1072.275849 \\ 770.616775 & 1550.486085 & -1072.455935 \\ 770.137064 & 1550.840529 & -1072.919218 \\ 769.705667 & 1551.597002 & -1073.277772 \\ 769.667907 & 1552.495141 & -1073.231095 \\ 769.620962 & 1552.899021 & -1072.841426 \\ 770.044574 & 1552.387264 & -1072.355602 \\ 770.426979 & 1551.897803 & -1072.140927 \\ 770.579473 & 1551.292038 & -1072.308221 \\ 770.790747 & 1550.625984 & -1072.311913 \\ 770.351972 & 1551.015693 & -1072.745408 \\ 769.938660 & 1551.604850 & -1073.089681 \\ 769.667537 & 1552.335897 & -1073.233661 \\ 769.559194 & 1552.717889 & -1072.844626 \\ 769.969812 & 1552.338095 & -1072.426043 \\ 770.353091 & 1551.801435 & -1072.163639 \\ 770.437077 & 1551.137935 & -1072.381601 \\ 770.748777 & 1550.433674 & -1072.273395 \\ 770.312096 & 1550.874401 & -1072.694122 \\ 769.799915 & 1551.473179 & -1073.120961 \\ 769.487891 & 1552.135257 & -1073.315887 \\ 769.477775 & 1552.697316 & -1072.916869 \\ 769.921150 & 1552.318997 & -1072.448942 \\ 770.342649 & 1551.788632 & -1072.170431 \\ 770.534507 & 1551.130885 & -1072.273409 \\ 770.871600 & 1550.643714 & -1072.241673 \\ 770.429076 & 1551.090447 & -1072.676286 \\ 769.914130 & 1551.635197 & -1073.138502 \\ 769.509611 & 1552.317202 & -1073.371535 \\ 769.518032 & 1552.877008 & -1072.955832 \\ 769.950891 & 1552.516358 & -1072.466577 \\ 770.371810 & 1551.988918 & -1072.209229 \\ 770.535588 & 1551.338018 & -1072.368815 \\ 770.829730 & 1550.657179 & -1072.276041 \\ 770.400691 & 1551.085855 & -1072.718035 \\ 769.904694 & 1551.653545 & -1073.134964\end{array}$

Table 4.8 Robot position resolution analysis results (commanded incremental moves of $0.5 \mathrm{~mm}$ ). 
769.550576

769.528740

769.972289

770.356424

770.488519

770.788064

770.362193

769.859391

769.462330

769.511870

769.926480

770.361831

770.544471

770.847983

770.428509

769.921437

769.498539
1552.259359

1552.72298

1552.350940

1551.895099

1551.202329

1550.513671

1550.933777

1551.511742

1552.121523

1552.669589

1552.354113

1551.851081

1551.204854

1550.519134

1550.916555

1551.474946

1552.111628

Movement in the Z-Axis direction

$\begin{array}{lll}769.433704 & 1552.520788 & -1073.001668 \\ 769.359997 & 1552.294272 & -1073.143947 \\ 769.355270 & 1552.326698 & -1073.624622 \\ 769.519639 & 1552.158798 & -1073.757073 \\ 769.572665 & 1552.095362 & -1074.193889 \\ 769.568668 & 1552.318161 & -1074.240185 \\ 769.571584 & 1552.465165 & -1073.921433 \\ 769.688959 & 1552.802102 & -1073.542023 \\ 769.906065 & 1552.784124 & -1072.685931 \\ 769.866167 & 1552.652340 & -1072.795295 \\ 769.782058 & 1552.742035 & -1073.351839 \\ 769.703893 & 1552.433098 & -1073.684952 \\ 769.465698 & 1552.058782 & -1074.272030 \\ 769.408818 & 1552.222223 & -1074.317985 \\ 769.474270 & 1552.366504 & -1073.957806 \\ 769.589189 & 1552.685837 & -1073.601756 \\ 769.637005 & 1552.742075 & -1072.878837 \\ 769.601692 & 1552.613512 & -1072.960084 \\ 769.508066 & 1552.658902 & -1073.521923 \\ 769.465451 & 1552.325754 & -1073.818527 \\ 769.317733 & 1552.031860 & -1074.350854 \\ 769.273778 & 1552.201392 & -1074.393450 \\ 769.273864 & 1552.365666 & -1074.107382 \\ 769.406282 & 1552.686632 & -1073.738304 \\ 769.709209 & 1552.748934 & -1072.872415 \\ 769.696715 & 1552.625483 & -1072.905854 \\ 769.579730 & 1552.678687 & -1073.478285 \\ 769.596191 & 1552.382512 & -1073.740383 \\ 769.557481 & 1552.013377 & -1074.186928 \\ 769.503762 & 1552.188945 & -1074.203798 \\ 769.497201 & 1552.352689 & -1073.946330 \\ 769.565165 & 1552.702092 & -1073.616496 \\ 769.840621 & 1552.780586 & -1072.753952 \\ 769.856038 & 1552.602015 & -1072.778013\end{array}$

Table 4.8 Robot position resolution analysis results (commanded incremental moves of $0.5 \mathrm{~mm}$ ). 


$\begin{array}{lll}769.732632 & 1552.653199 & -1073.382687 \\ 769.653767 & 1552.364817 & -1073.709379 \\ 769.550292 & 1552.045477 & -1074.180222 \\ 769.460403 & 1552.222444 & -1074.239289 \\ 769.468064 & 1552.346013 & -1073.972353 \\ 769.570478 & 1552.649251 & -1073.630228 \\ 769.690804 & 1552.765159 & -1072.884378 \\ 769.636112 & 1552.675951 & -1072.972767 \\ 769.547664 & 1552.705261 & -1073.479659 \\ 769.548180 & 1552.354671 & -1073.773899 \\ 769.393213 & 1552.087881 & -1074.310423 \\ 769.344663 & 1552.273207 & -1074.331390 \\ 769.331633 & 1552.404125 & -1074.072872 \\ 769.413670 & 1552.720594 & -1073.734059 \\ 769.690657 & 1552.811272 & -1072.892284 \\ 769.698957 & 1552.662814 & -1072.908828 \\ 769.606612 & 1552.703711 & -1073.450457 \\ 769.628832 & 1552.394758 & -1073.741330 \\ 769.565476 & 1552.085762 & -1074.177185 \\ 769.546244 & 1552.251706 & -1074.199763 \\ 769.538448 & 1552.367693 & -1073.922414 \\ 769.560115 & 1552.721383 & -1073.646588\end{array}$

Table 4.8 Robot position resolution analysis results (commanded incremental moves of $0.5 \mathrm{~mm}$ ).

MEAN VALUE AND STANDARD DEVIATION OF THE RESOLUTION IN THE X-AXIS DIRECIION

Resolution $=0.594424$ Sigma $=0.160903$

MEAN VALUE AND STANDARD DEVIATION OF THE RESOLUTION IN THE Y-AXIS DIRECTION

Resolution $=0.732857$ Sigma $=0.094315$

MEAN VALUE AND STANDARD DEVIATION OF THE RESOLUTION IN THE Z-AXIS DIRECTION

Resolution $=0.440251$ Sigma $=0.213103$ 
Table 4.9 Forward kinematics error analysis results (continuous operation).

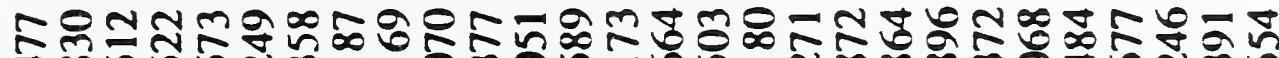

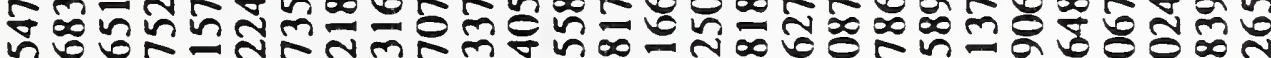
ผ ㄷํำำซ

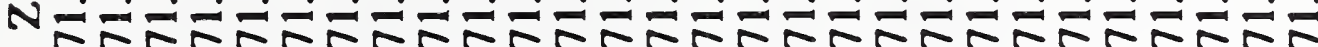

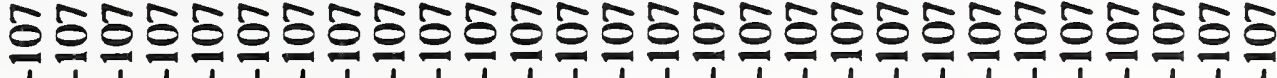

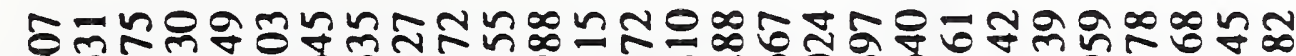

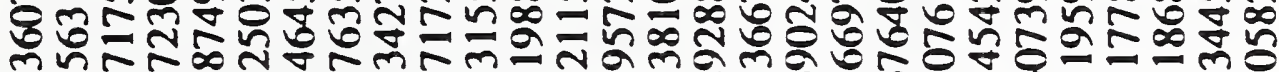
สำ

خ

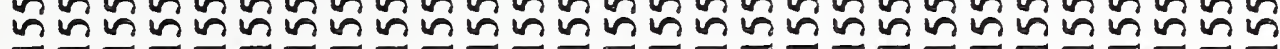

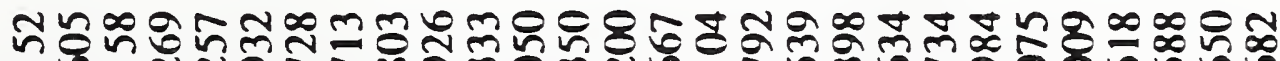

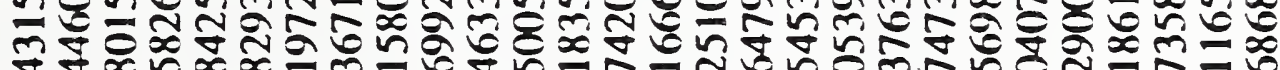

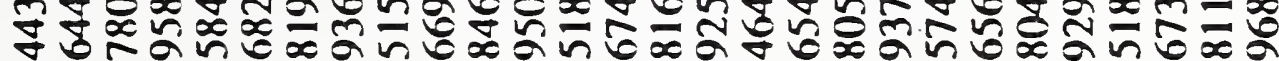

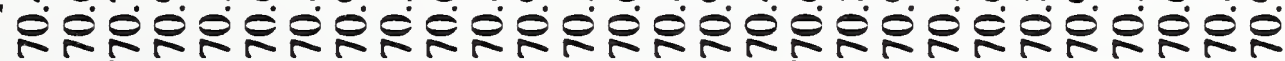

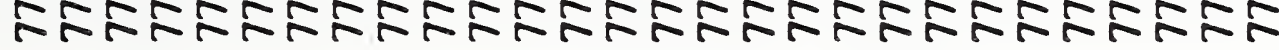

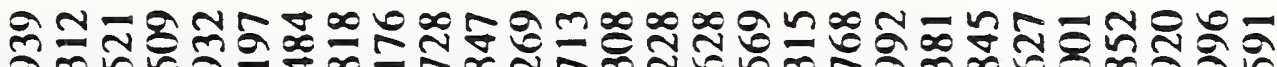
ฝิ

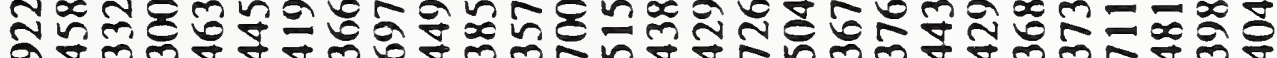
$\mathrm{N}$

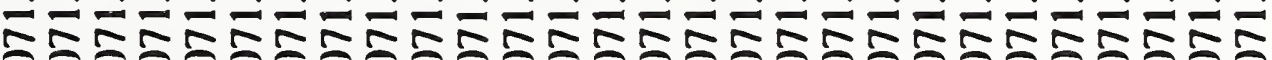

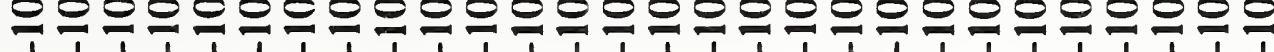

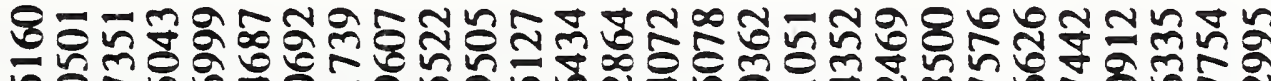

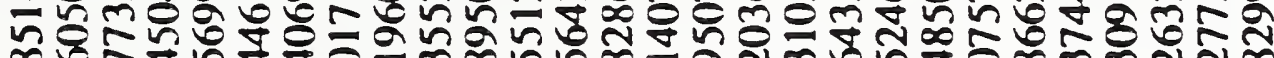

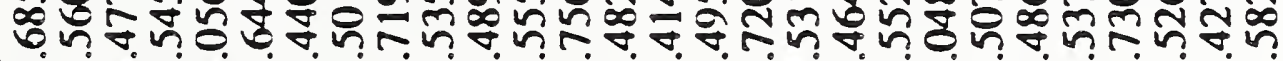

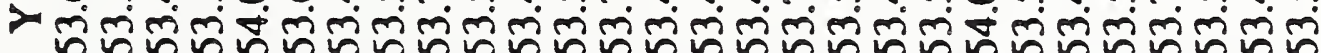

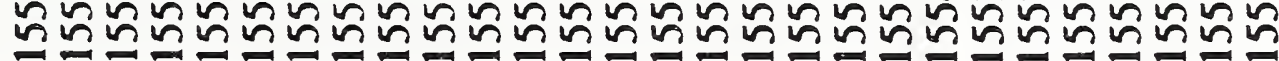

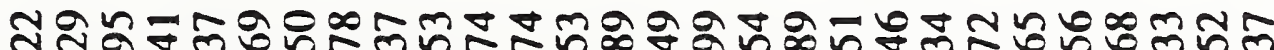

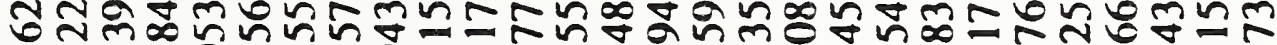

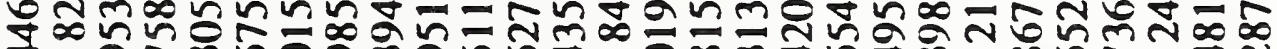
শন্য়

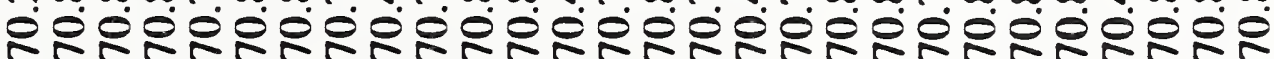

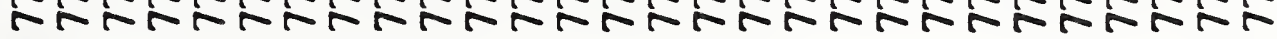


Table 4.9 Forward kinematics error analysis results (continuous operation).

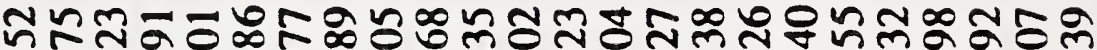

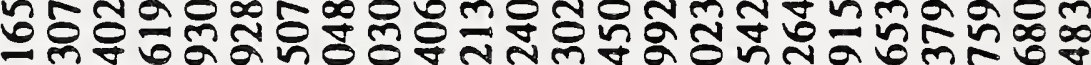

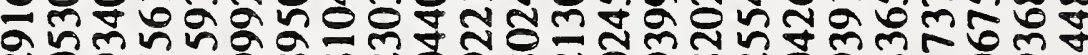

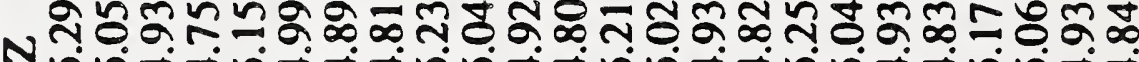

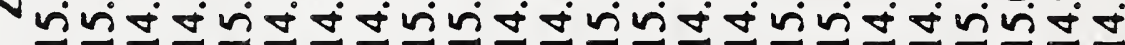
б.

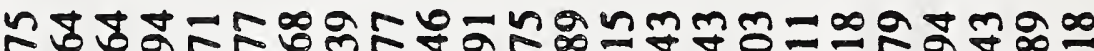

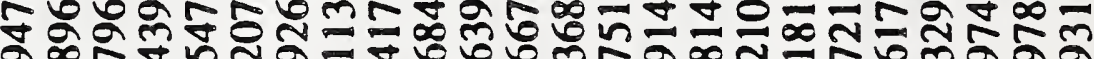
운

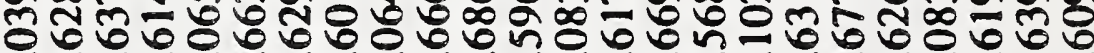

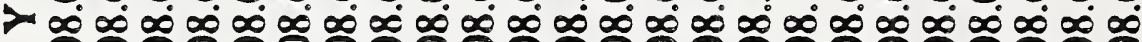

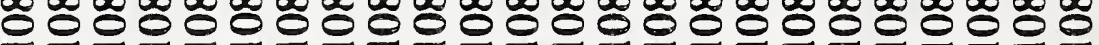

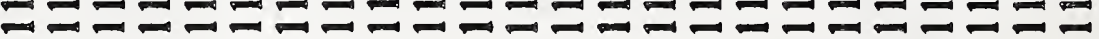

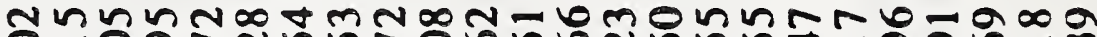

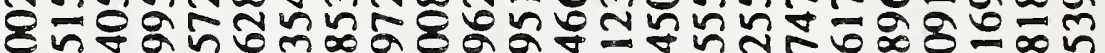

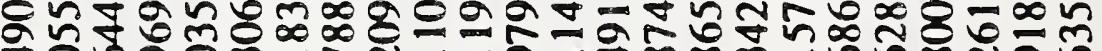

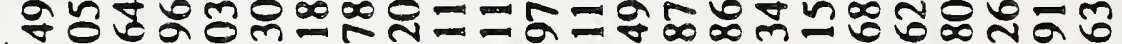
x

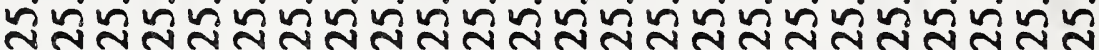

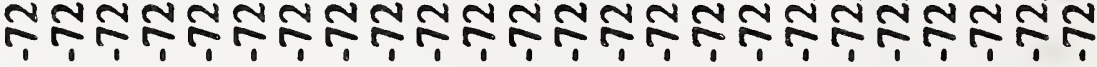

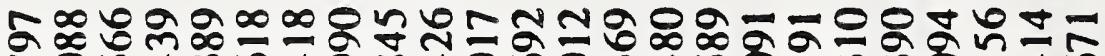
윤

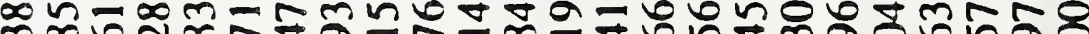

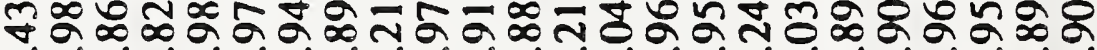

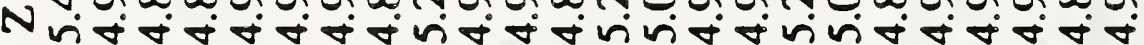
б.

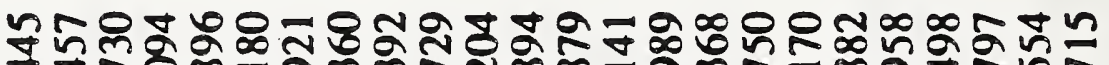

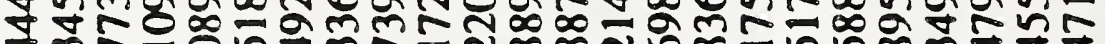
ZुK

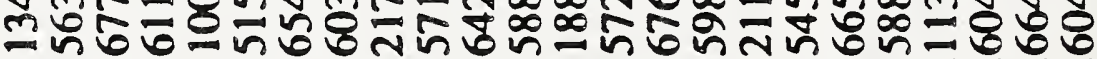

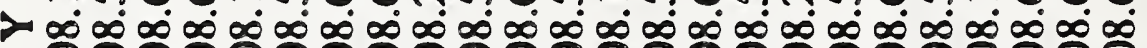

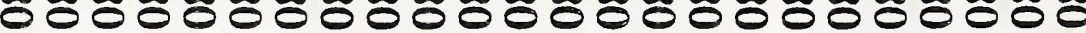

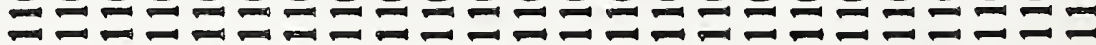

๒ูป

$\nabla \approx$ जิ

mีn

$\infty$ no

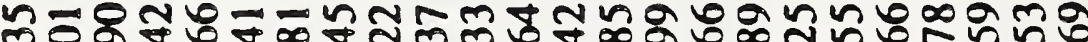

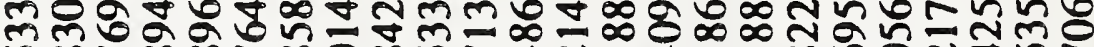

जิ 疋 vทंที่

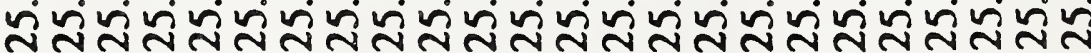

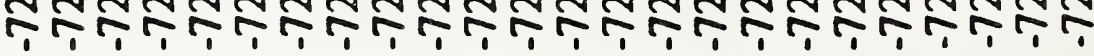


Table 4.9 Forward kinematics error analysis results (continuous operation).

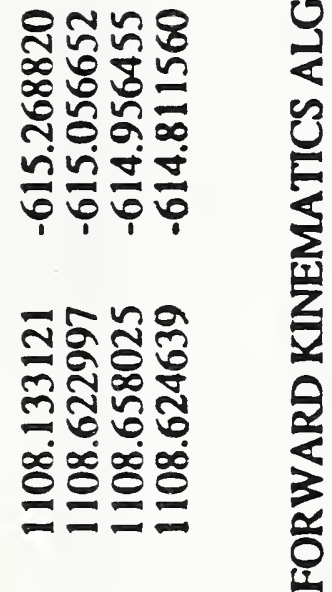

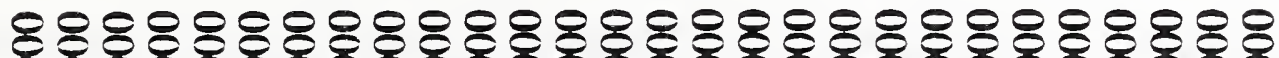

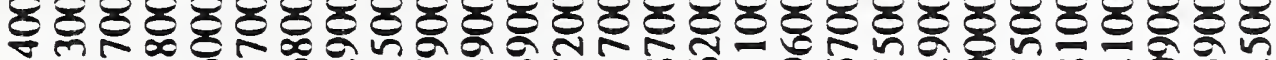

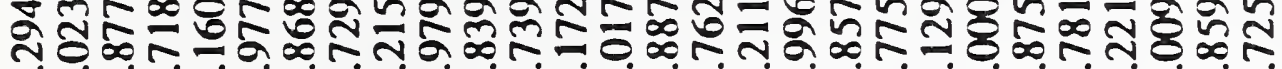

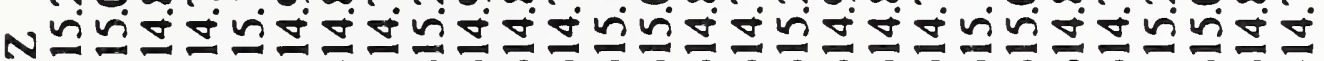

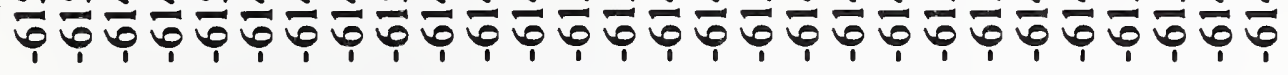

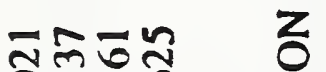

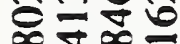

我要

งที่ง

กำ

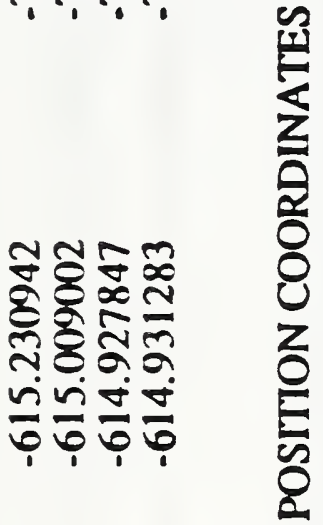

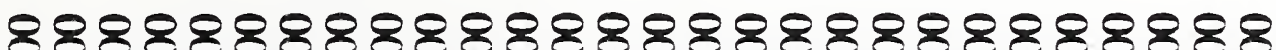

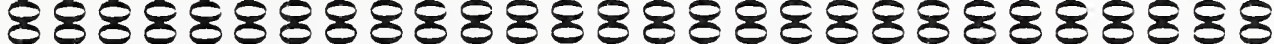

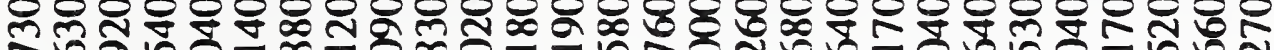

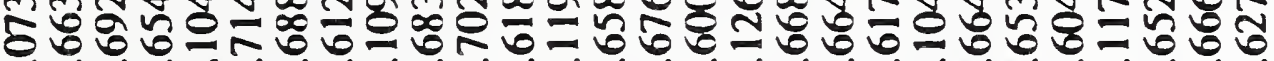
入

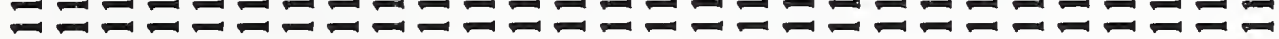

คำำ ทำ ํํํ $6 \infty=2$ 은 $\ddot{0} \infty \ddot{0} \infty$ $=0$

రํำ ㅇํㅇำ त. 幽每 ฉที่ कึतส

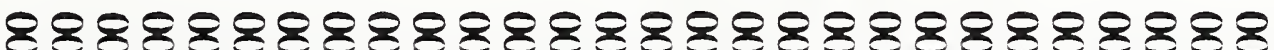

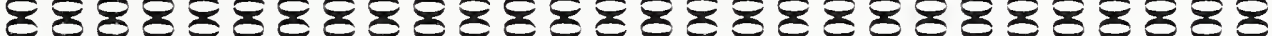

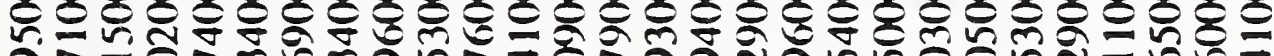
คิล

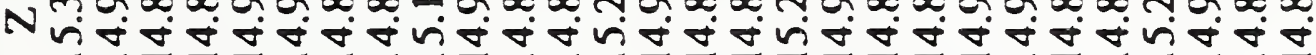

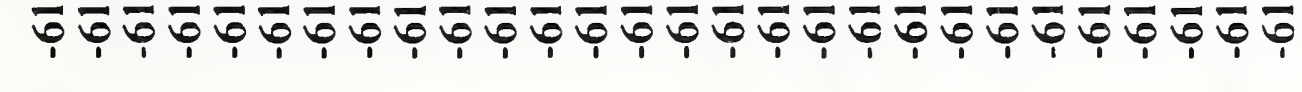

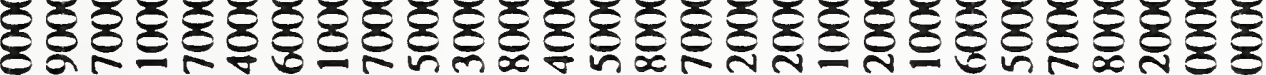

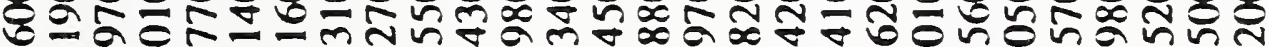

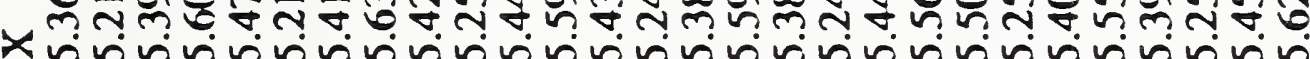

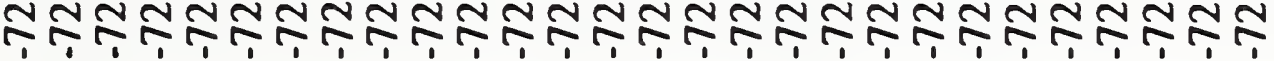

888888888888888888888888888\%

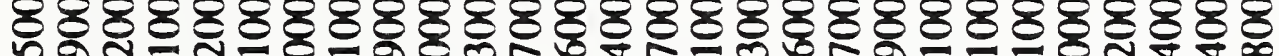
次 न๗

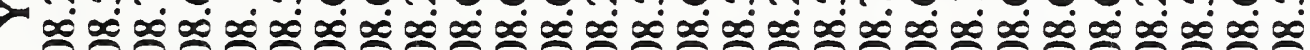

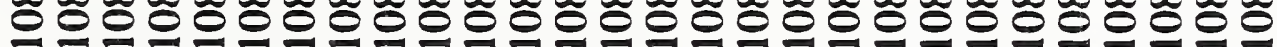

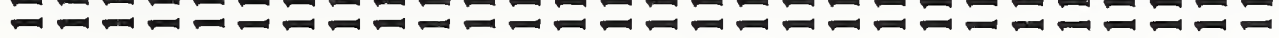

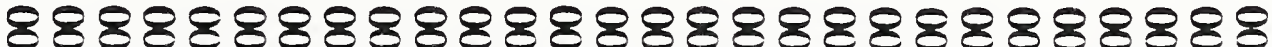

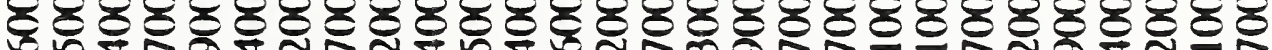

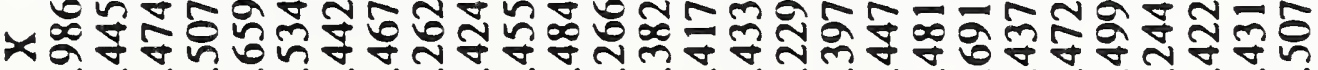

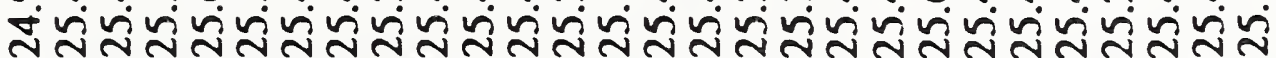

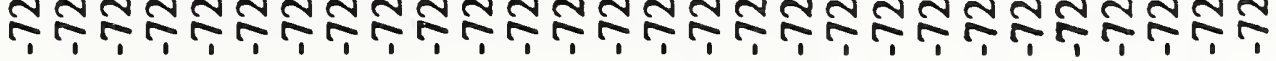


Table 4.9 Forward kinematics error analysis results (continuous operation).

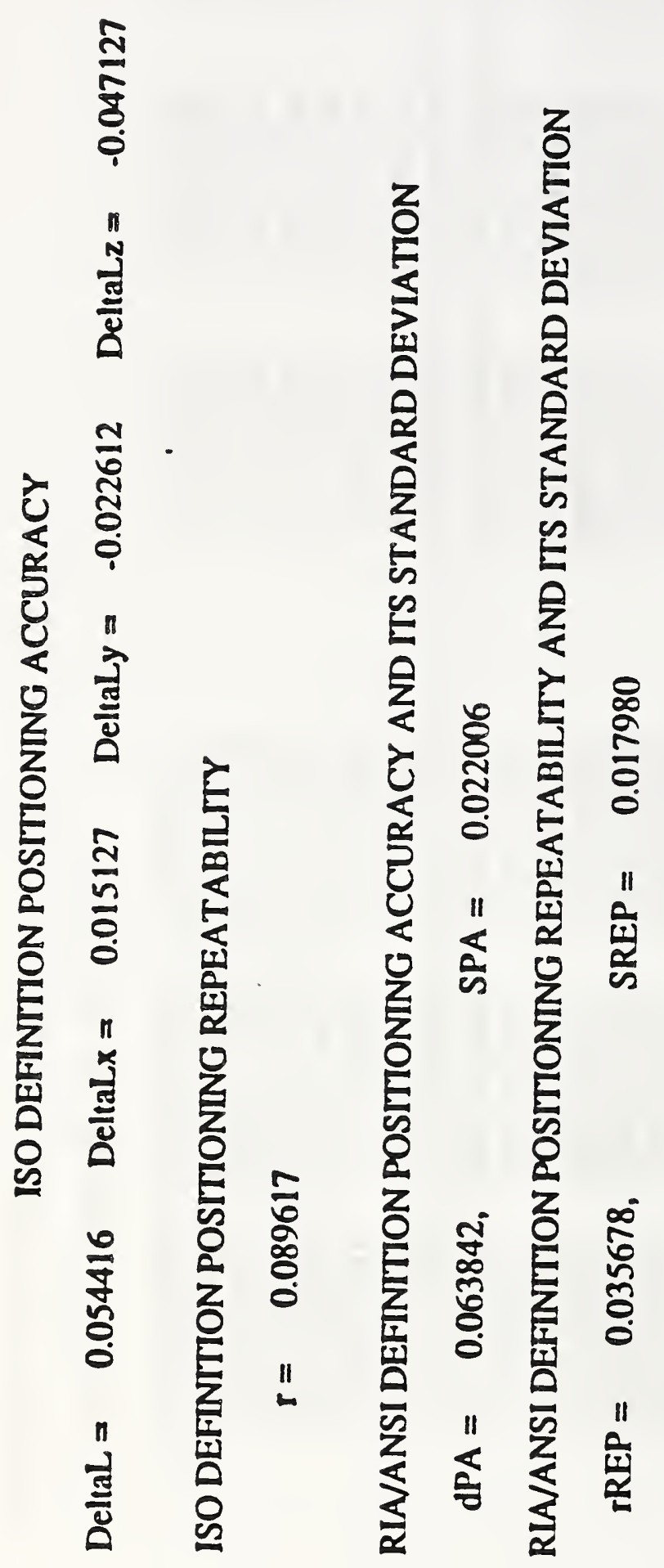


Table 4.10 Forward kinematics error analysis results (interrupted operation).

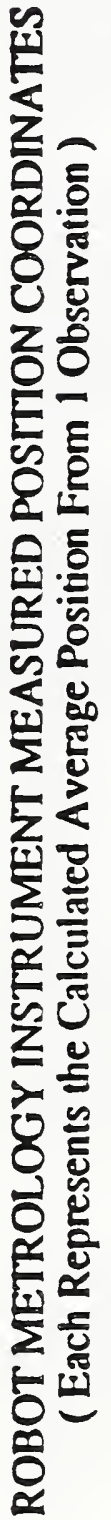

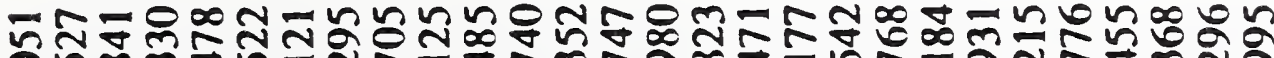
ㄴํㅇ

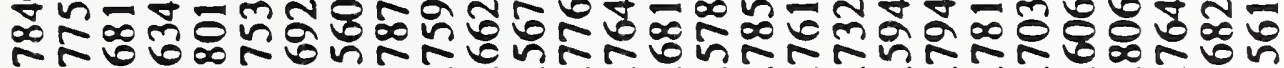

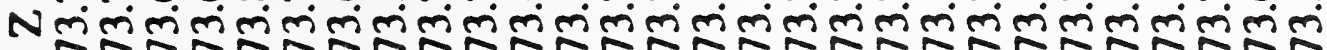
5555555555555555555555555555

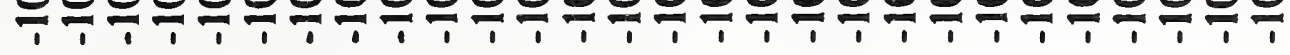

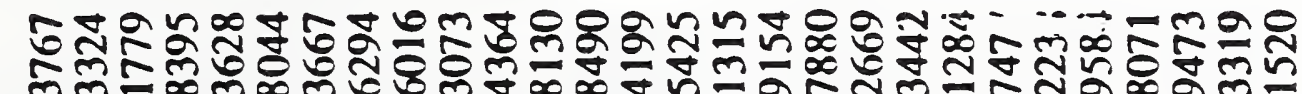
ஸต

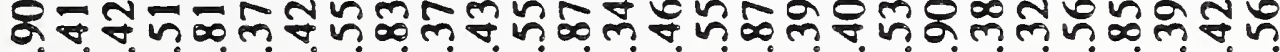

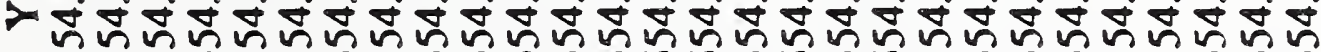

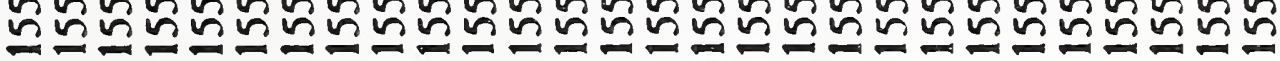

ำต์

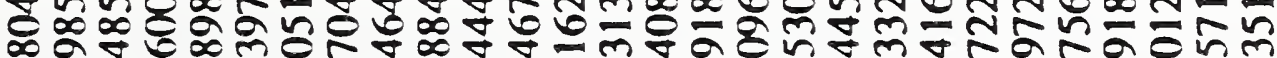

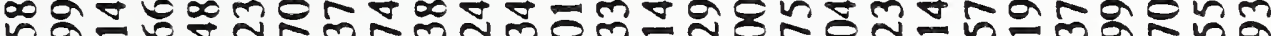
x レீ

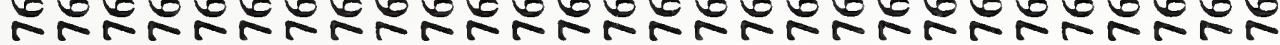

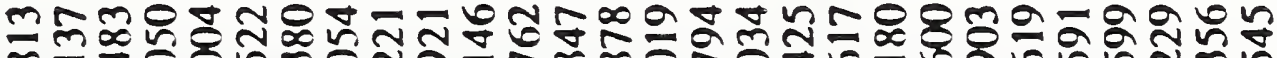

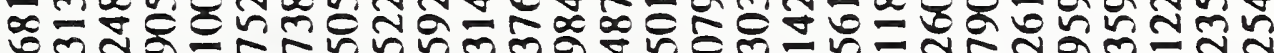

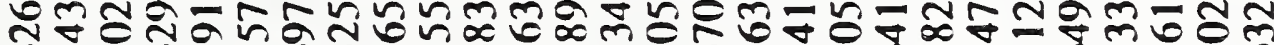

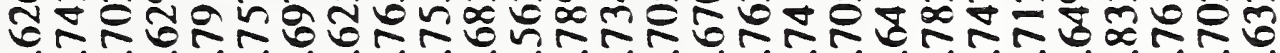

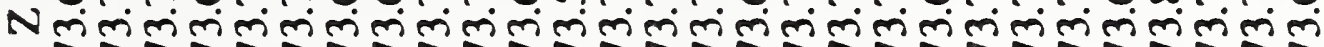

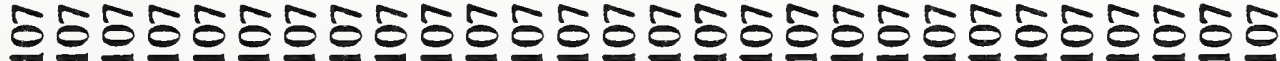

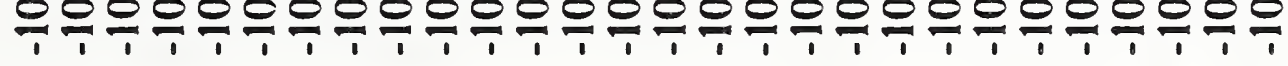

ஸิษ

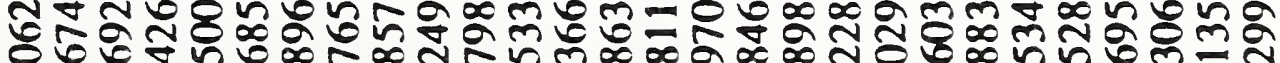
웅

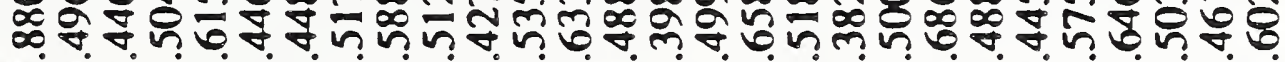

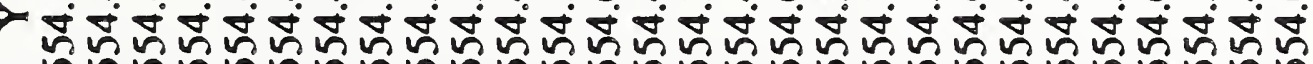

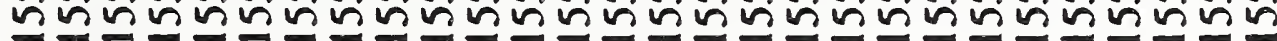

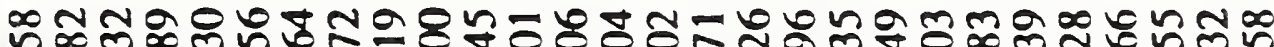

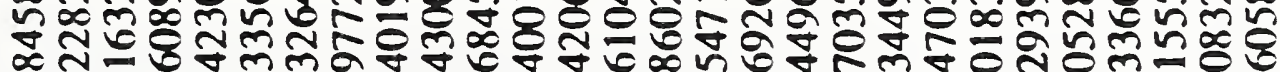
중

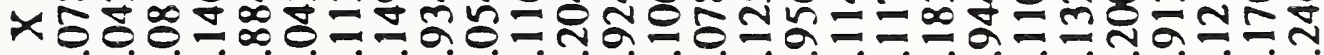
ษ

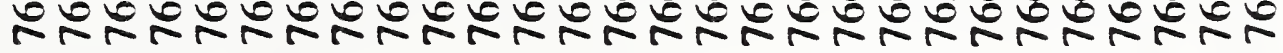


Table 4.10 Forward kinematics error analysis results (interrupted operation).

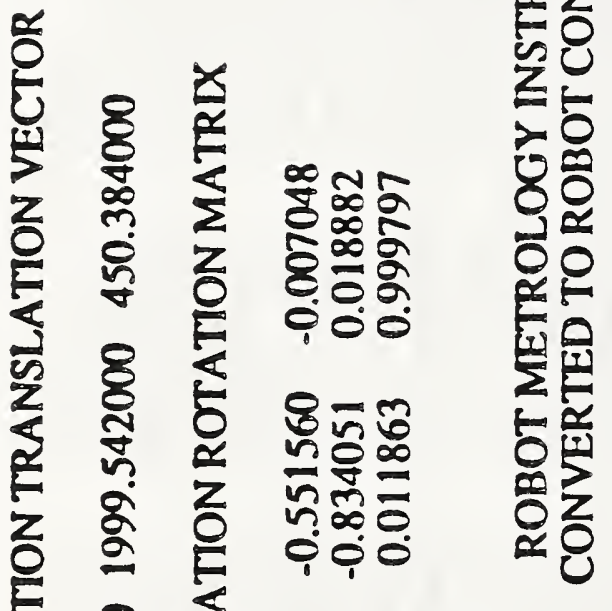

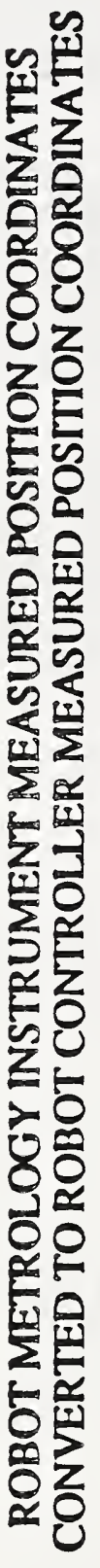

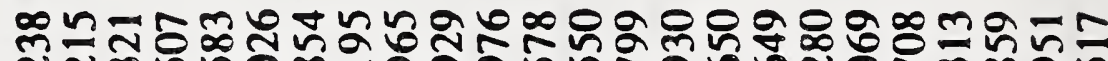

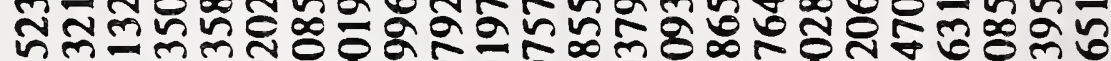

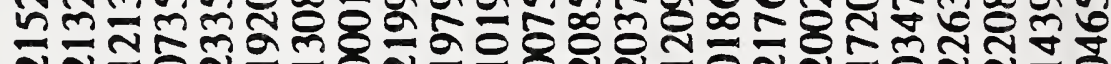

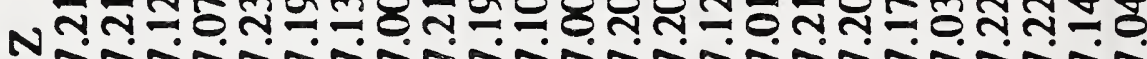

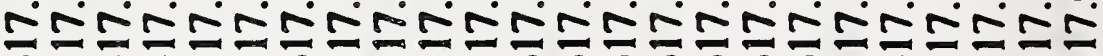

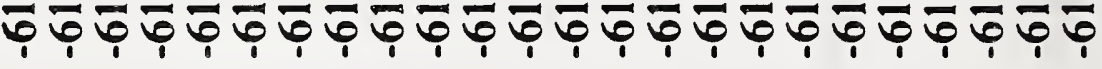

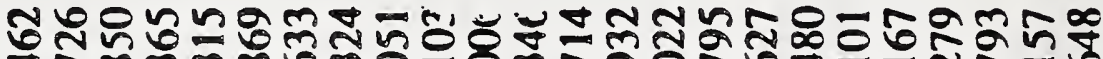
중ำ

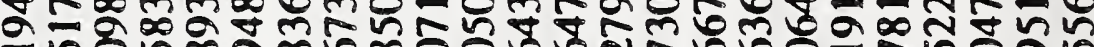

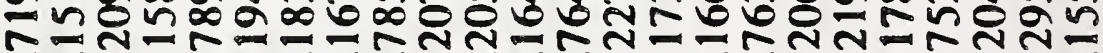

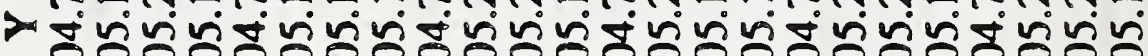

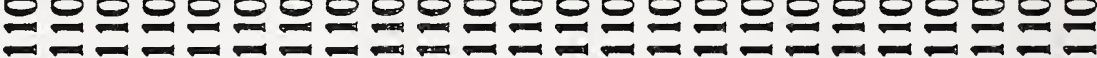

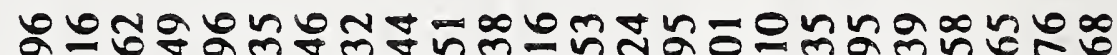

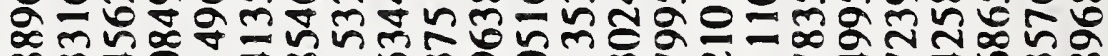

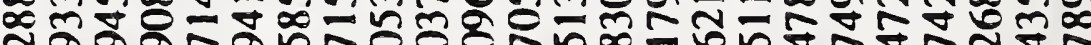

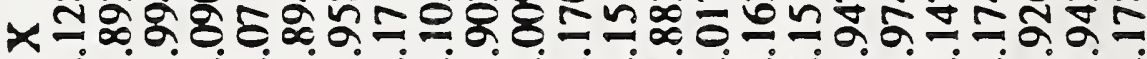

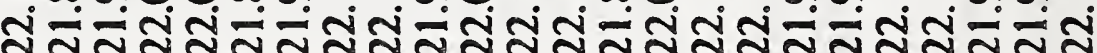
N

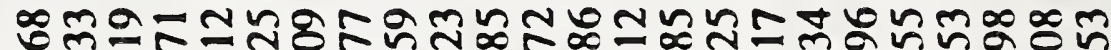

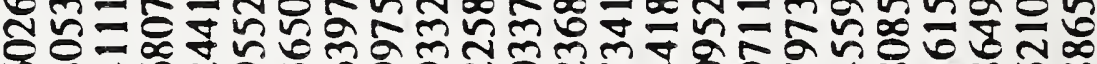

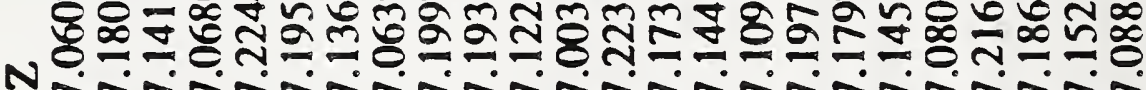

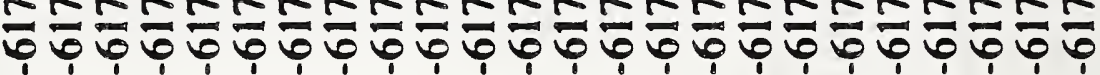

กี่

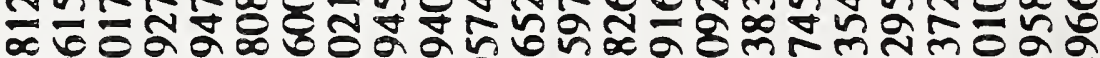

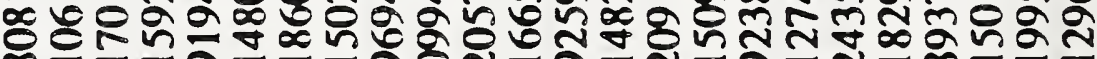
-

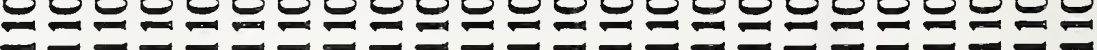

एᄂ?

$\forall \underset{ }{ }$

m

ஜீं

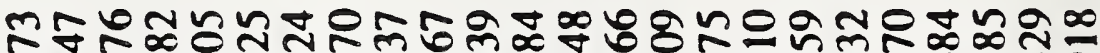

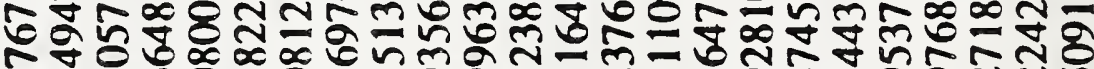

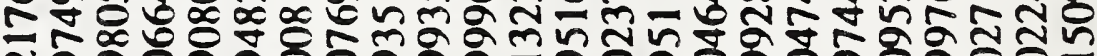

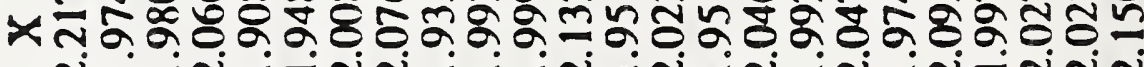

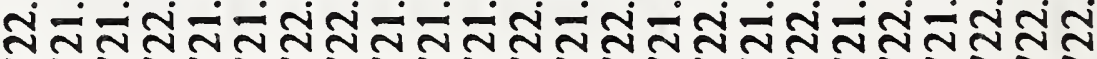

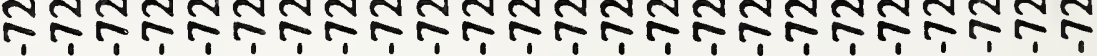


Table 4.10 Forward kinematics error analysis results (interrupted operation).

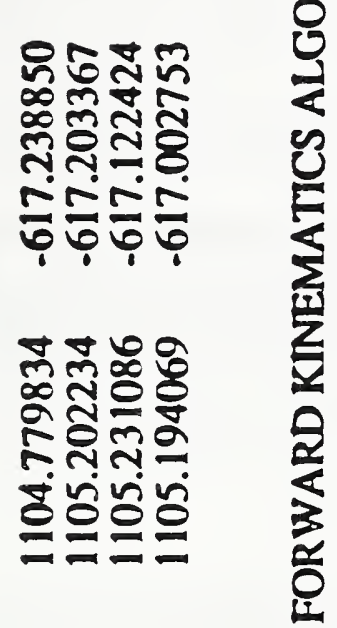

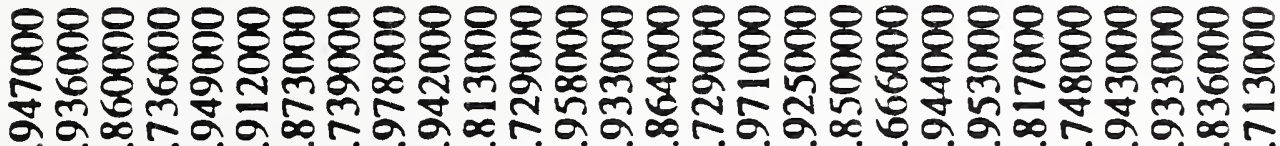

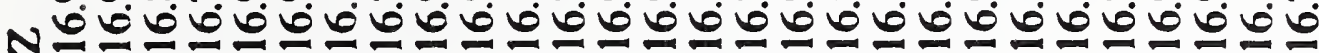

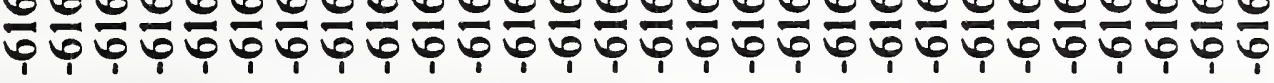

$\infty$ 용요

$\infty$

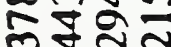

ติอง

กิ่งกิร

กับง

TิT

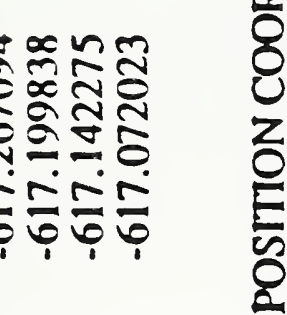

$\sim \in N \infty$

$\infty \approx n \frac{1}{2}$

$8 \pi \operatorname{mon}$

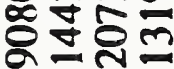

$\sum^{\circ} 0^{\circ}{ }^{\circ}$

ำกิกีอ

원ำ

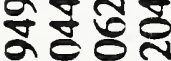

เง

ำกิร

年

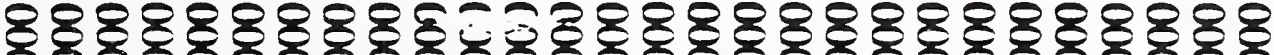

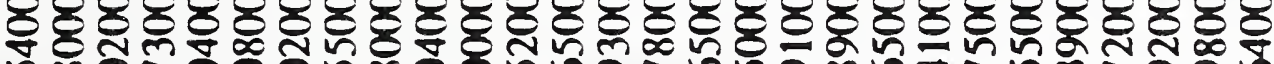

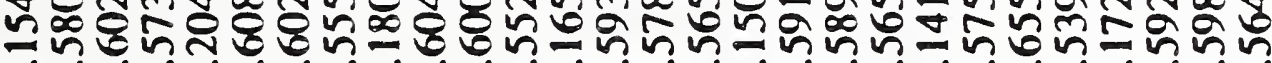

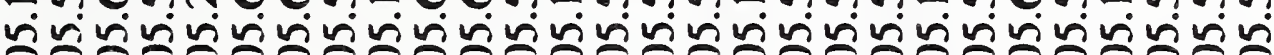

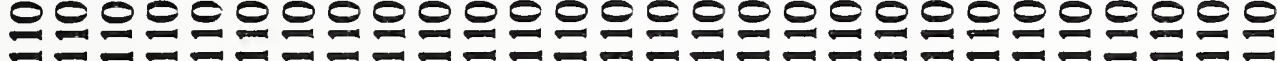

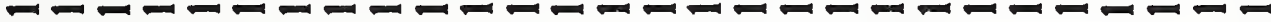

8888888888888888888888888888

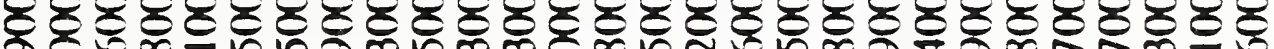

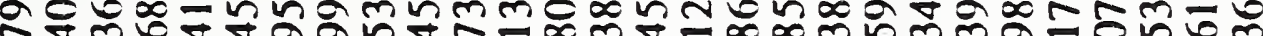

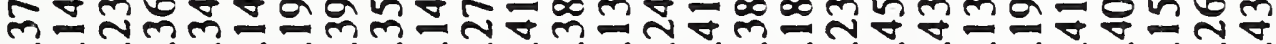
×

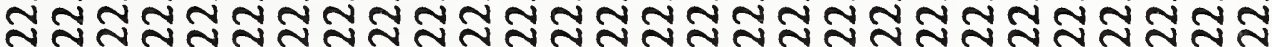
TTTTTTTTTTTTTTTTTTTTTTT

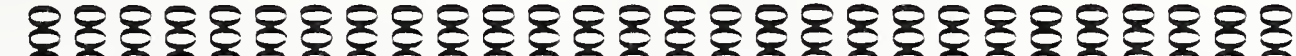

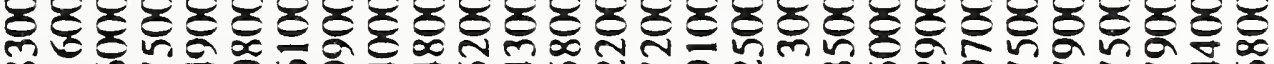

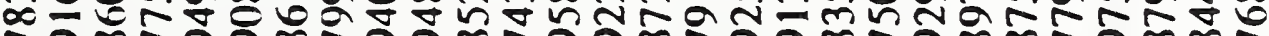
N

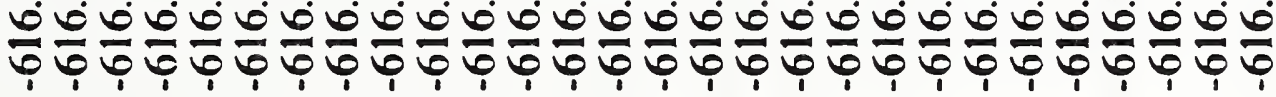

8888888888888888888888888888

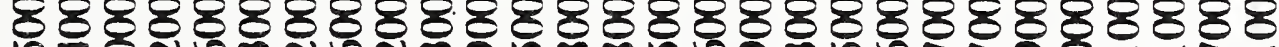
传

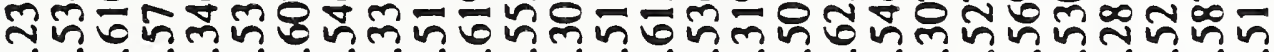
vivinunvinunvinunvinunvinu 응ㅇㅇㅇㅇㅇㅇㅇㅇㅇㅇㅇㅇㅇㅇㅇㅇㅇㅇㅇㅇㅇㅇㅇㅇㅇㅇㅇㅇㅇㅇㅇㅇㅇㅇㅇㅇㅇ으응

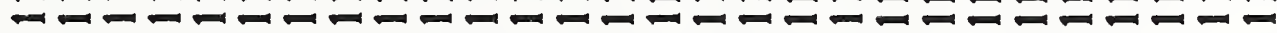

8888888888888888888888888888

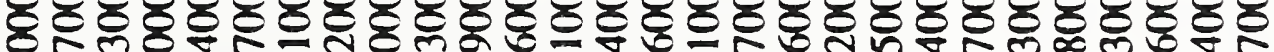
×赵

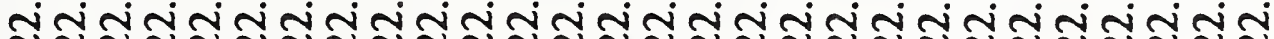

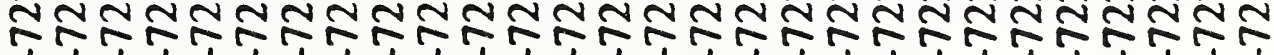


Table 4.10 Forward kinematics error analysis results (interrupted operation).

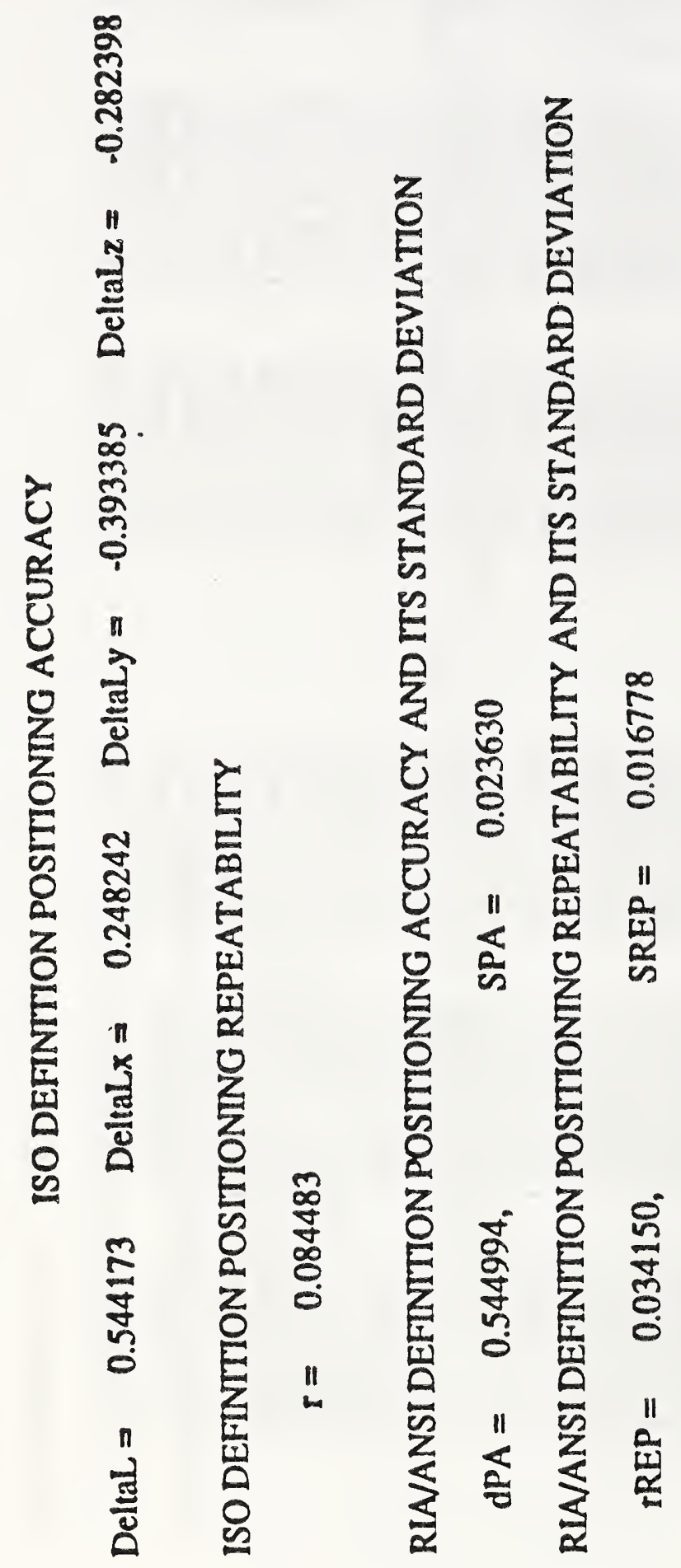




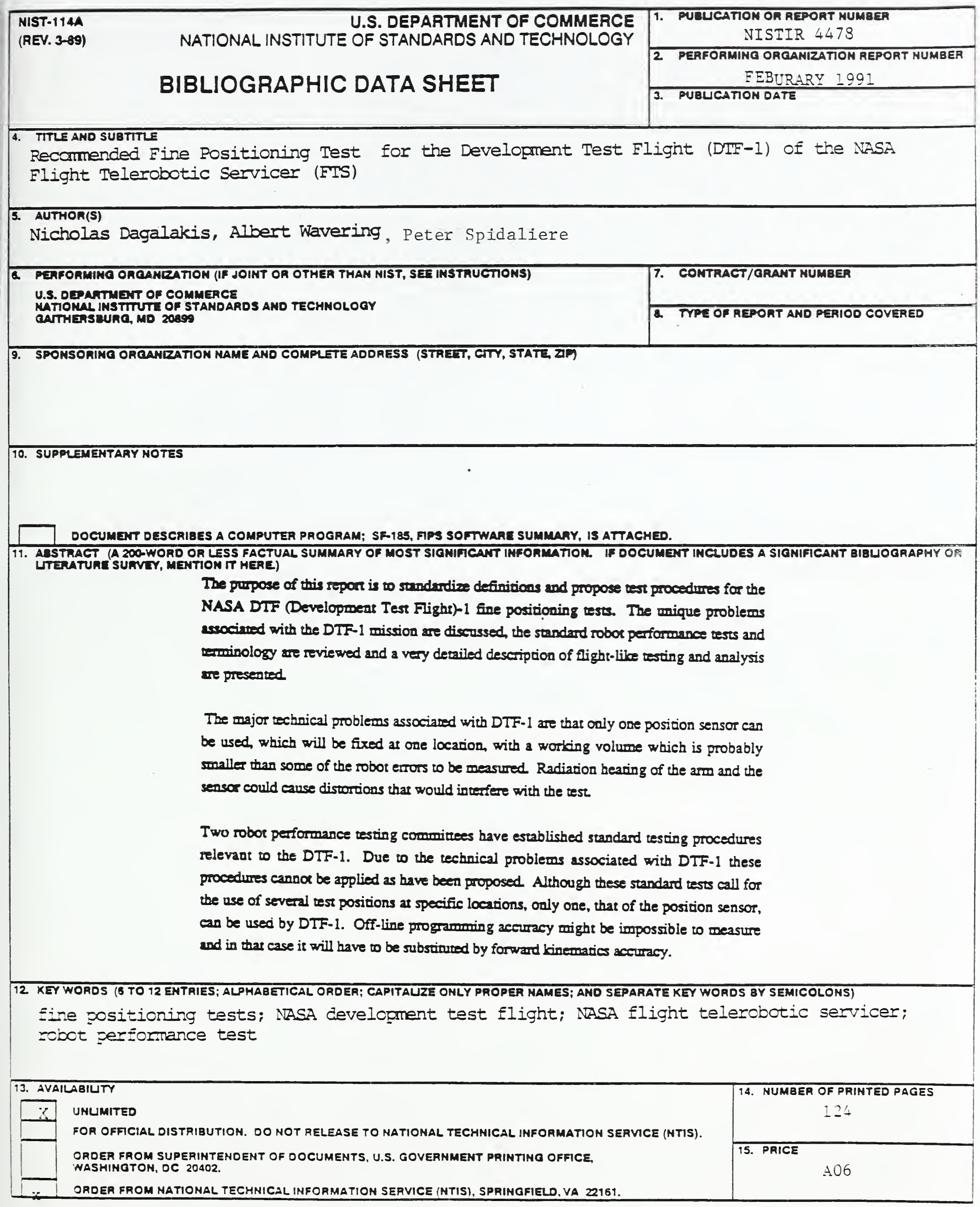




